University of Louisville

ThinkIR: The University of Louisville's Institutional Repository

Electronic Theses and Dissertations

8-2014

\title{
A novel NMF-based DWI CAD framework for prostate cancer.
}

Patrick McClure

University of Louisville

Follow this and additional works at: https://ir.library.louisville.edu/etd

Part of the Computer Engineering Commons

\section{Recommended Citation}

McClure, Patrick, "A novel NMF-based DWI CAD framework for prostate cancer." (2014). Electronic Theses and Dissertations. Paper 936.

https://doi.org/10.18297/etd/936

This Master's Thesis is brought to you for free and open access by ThinkIR: The University of Louisville's Institutional Repository. It has been accepted for inclusion in Electronic Theses and Dissertations by an authorized administrator of ThinkIR: The University of Louisville's Institutional Repository. This title appears here courtesy of the author, who has retained all other copyrights. For more information, please contact thinkir@louisville.edu. 


\title{
A NOVEL NMF-BASED DWI CAD FRAMEWORK FOR PROSTATE CANCER
}

\author{
By \\ Patrick McClure \\ B.S. in Bioengineering, University of Louisville, USA, 2013
}

\begin{abstract}
A Thesis
Submitted to the Faculty of the

J.B. Speed School of Engineering of the University of Louisville in Partial Fulfillment of the Requirements

for the Degree of

Master of Science

Department of Computer Engineering and Computer Science University of Louisville

Louisville, Kentucky
\end{abstract}

August 2014 



\title{
A NOVEL NMF-BASED DWI CAD FRAMEWORK FOR PROSTATE CANCER
}

\author{
By \\ Patrick McClure \\ B.S. in Bioengineering, University of Louisville, USA, 2013
}

A Thesis Approved On

$\frac{05 / 30 / 2014}{\text { Date }}$

by the following Thesis Committee:

Adel Elmaghraby, Ph.D., Thesis Director

Ayman El-Baz, Ph.D., Thesis Co-Director

Olfa Nasraoui, Ph.D. 


\section{ACKNOWLEDGEMENTS}

Above all, I would like to thank the Lord God for giving me the opportunity and abilities to be successful in this endeavour. Throughout my life and academic career, countless people have supported me. I would like to briefly acknowledge a select few who have had the most impact. Foremost, I want to thank my father, Lt. Col. Rick McClure, my mother, Juliana McClure, my brother, Christopher McClure, and my sister, Kristina McClure, for their continual love and support. Specifically, I want to thank my parents. None of this would have been possible without their teaching and guidance.

I would also like to thank my thesis co-advisor, Dr. Ayman El-Baz. Throughout the past four years, he has given me many opportunities to learn and excel. He has constantly supported me in my studies and made my academic success possible. I will always be indebted to him for his mentorship throughout my bachelors degree, masters degree, and, hopefully, the rest of my career. I also want to thank the rest my thesis committee, Dr. Olfa Nasraoui and Dr. Adel Elmaghraby, my CECS thesis advisor. Specifically, I want to thank Dr. Nasraoui for teaching me concepts in machine learning and optimization. I have used these skills in this thesis and will continue to use them. In addition, I want to thank Dr. Elmaghraby for helping me earn a MS in CS.

Additionally, I would like to thank Dr. Melvin Maron. He has been a mentor, colleague, and friend since I started studying at the University of Louisville. His mentorship, teaching, and encouragement have been invaluable. Finally, I would like to thank all of the BioImaging Lab members, Fahmi Khalifa, Ahmed Elnakib, Matthew Nitzken, Ahmed Soliman, Amir Alansary, and Mahmoud Mostapha. 
They have assisted me in writing publications, writing code, and solving problems in general throughout college. This research could not have been conducted without their support. 


\begin{abstract}
A NOVEL NMF-BASED DWI CAD FRAMEWORK FOR PROSTATE CANCER
\end{abstract}

Patrick McClure

August 12, 2014

In this thesis, a computer aided diagnostic (CAD) framework for detecting prostate cancer in DWI data is proposed. The proposed CAD method consists of two frameworks that use nonnegative matrix factorization (NMF) to learn meaningful features from sets of high-dimensional data. The first technique, is a three dimensional (3D) level-set DWI prostate segmentation algorithm guided by a novel probabilistic speed function. This speed function is driven by the features learned by NMF from 3D appearance, shape, and spatial data. The second technique, is a probabilistic classifier that seeks to label a prostate segmented from DWI data as either alignat, contain cancer, or benign, containing no cancer. This approach uses a NMF-based feature fusion to create a feature space where data classes are clustered. In addition, using DWI data acquired at a wide range of b-values (i.e. magnetic field strengths) is investigated. Experimental analysis indicates that for both of these frameworks, using NMF producing more accurate segmentation and classification results, respectively, and that combining the information from DWI data at several b-values can assist in detecting prostate cancer. 


\section{TABLE OF CONTENTS}

Page

ACKNOWLEDGEMENTS

ABSTRACT $\quad$ v

LIST OF TABLES viii

LIST OF FIGURES ix

\section{CHAPTER}

$\begin{array}{lll}\text { I INTRODUCTION } & \mathbf{1}\end{array}$

A In-Vitro Prostate Cancer Diagnostic Technologies . . . . . . . 4

B In-vivo Prostate Cancer Diagnostic Techniques . . . . . . . . . . 22

1 TRUS-based CAD systems . . . . . . . . . . . 26

2 MRI-based CAD systems . . . . . . . . . . 40

C Discussion ....................... 69

1 Potentials and limitations of the current prostate diagnostic techniques . . . . . . . . . . . 70

2 Research Challenges ............. . . 71

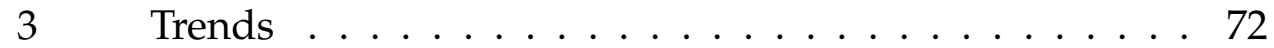



\section{A NOVEL NMF GUIDED LEVEL-SET FOR DWI PROSTATE SEG-}

$\begin{array}{ll}\text { MENTATION } & 74\end{array}$

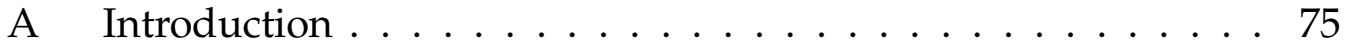

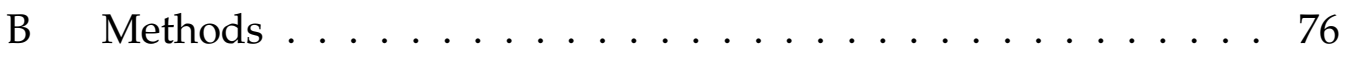

1 3D Appearance, Shape, and Spatial Features . . . . . . 77

2 NMF-based Feature Fusion . . . . . . . . . . . 80 
3 Estimation of the Stochastic Speed Function . . . . . 82

C Performance Metrics . . . . . . . . . . . . . . . . . 83

1 Dice Similarity Coefficient (DSC) . . . . . . . . . . 83

2 Hausdorf Distance (HD) . . . . . . . . . . . . . . 84

D Experimental Results . . . . . . . . . . . . . . . . . 85

$1 \quad$ Medical Images . . . . . . . . . . . . . . . . . 85

2 Segmentation Results . . . . . . . . . . . . . 86

E Conclusion ..................... . 89

III A NOVEL NMF-BASED DWI PROSTATE CANCER DETECTION $\begin{array}{ll}\text { FRAMEWORK } & 91\end{array}$

A Introduction . . . . . . . . . . . . . . . . . 91

B Methods ........................992

$1 \quad \mathrm{NMF} \ldots \ldots \ldots \ldots \ldots 2 \ldots \ldots \ldots$

2 Classification ................. . 93

3 Refinement . . . . . . . . . . . . . 95

C Results .................... 96

D Conclusion ................... 97

IV CONCLUSION AND FUTURE WORK 98

REFERENCES 100

$\begin{array}{ll}\text { CURRICULUM VITAE } & 149\end{array}$ 


\section{LIST OF TABLES}

TABLE

Page

1 Summary of in-vitro studies for diagnosing prostate cancer . . . . . . 12

2 Summary of in vivo studies for prostate segmentation from TRUS



3 Summary of prostate cancer CAD systems using in-vivo TRUS images 37

4 Summary of in vivo studies for prostate segmentation from MRI . . . 43

5 Summary of prostate cancer CAD systems using in-vivo MRI . . . . . 55

6 The DSC segmentation performances . . . . . . . . . . 88

7 The HD segmentation performances . . . . . . . . . . . 88

8 Comparison of the average DSC and HD values . . . . . . . . . 88

9 Comparison of the accuracies (ACCs) of classification approaches . . 96 


\section{LIST OF FIGURES}

FIGURE Page

1 Diagram of the three prostate zones ............ 3

2 Different clinically used in-vitro biomarkers . . . . . . . . . . . . 5

3 Typical CT, TRUS and T2-MR images of a prostate . . . . . . . . . 22

4 Different MR images of the prostate . . . . . . . . . . . 24

5 Diagram of a general fully-automated CAD system for prostate cancer 26

6 A diagram of the proposed segmentation framework. . . . . . . . 76

7 Illustration of a voxel's neighborhood . . . . . . . . . . 78

8 Diagram illustrating the meaning of TP, FP, TN and FP . . . . . . . 84

9 Diagram illustrating the 2 D HD . . . . . . . . . . . 85

10 Sample prostate segmentations for the proposed approach . . . . . . 86

11 Example prostate segmentations . . . . . . . . . . . . 87

12 Example 3D prostate segmentation visualizations . . . . . . . . 88

13 Sample ROC curve for the NMF and MAP guided level-set approaches 89

14 Diagram of the DWI NMF-based cancer detection framework . . . . 93

15 Example $\mathrm{H}$-space vectors using $W$ and $W^{T} \ldots \ldots \ldots$. . . . . . 94 


\section{CHAPTER I}

\section{INTRODUCTION}

This chapter overviews one of the most important, interesting, and challenging problems in oncology, early diagnosis of prostate cancer. Developing effective diagnostic techniques for prostate cancer is of great clinical importance and can improve the effectiveness of treatment and increase the patient's chance of survival. The main focus of this study is to overview the different in-vitro and in-vivo technologies for diagnosing prostate cancer. This review discusses the current clinically used in-vitro cancer diagnostic tools, such as biomarker tests and needle biopsies, including their applications, advantages, and limitations. In addition to the in-vitro techniques, the current study discusses in detail developed in-vivo non-invasive state-of-the-art Computer-Aided Diagnosis (CAD) systems for prostate cancer based on analyzing Transrectal Ultrasound (TRUS) and different types of magnetic resonance imaging (MRI), e.g., T2-MRI, Diffusion Weighted Imaging (DWI), Dynamic Contrast Enhanced (DCE)-MRI, and multi-parametric MRI, focusing on their implementation, experimental procedures, and reported outcomes. Furthermore, the chapter addresses the limitations of the current prostate cancer diagnostic techniques, outlines the challenges that these techniques face, and introduces the recent trends to solve these challenges.

\section{Introduction}

Prostate cancer is the second most fatal cancer experienced by American males [1]. The average American male has a 16.15\% chance of developing prostate 
cancer, which is $8.38 \%$ higher than lung cancer, the second most likely cancer [1]. Therefore, early detection of prostate cancer is crucial in decreasing prostate cancer related deaths [2]. Recent reports indicate that the mortality rate of prostate cancer has decreased by approximately 42\% between 1991 and 2005 [3]. Approximately $45 \%$ of this decrease is due to the increased use of screening techniques [4]. While in-vitro techniques that are based on analyzing a patient's blood, urine, or tissue samples are commonly used, they have several limitations concerning their accuracy and the invasive nature of most methods. Thus far, non-biopsy screening techniques, predominantly prostate specific antigen (PSA) blood-based screening [5], have a high chance of false positive diagnosis, ranging from $28 \%-58 \%$ [4]. More accurate, non-invasive diagnostic systems would aid clinicians in early detection of prostate cancer. To accomplish this, in-vivo computer aided diagnostic (CAD) systems have been developed to locate and to classify prostate tumors based on extracting information from medical images. The goal of this chapter is to overview common in-vitro and in-vivo techniques for prostate cancer. This includes the several types of in-vitro techniques such as biomarker tests and needle biopsies. In addition, this chapter overviews common techniques used in the three basic steps of start-of-the-art prostate cancer CAD systems developed throughout the last decade. These are prostate segmentation, feature extraction, and classification. Furthermore, several complete CAD systems for the diagnosis of prostate cancer, as well as their developed computational methods and reported experimental procedures, will be discussed. In order to introduce the related work for prostate cancer diagnosis, a brief overview of the anatomy and the function of the prostate is given below.

The prostate is the largest male accessory organ [6]. It surrounds the urethra as it exits the bottom of the bladder (see Fig. 1) [7]. The prostate is a gland with an approximately elliptical shape, an approximate width of $4 \mathrm{~cm}$, and approximate thickness of $3 \mathrm{~cm}$, although the size varies widely. [6]. 


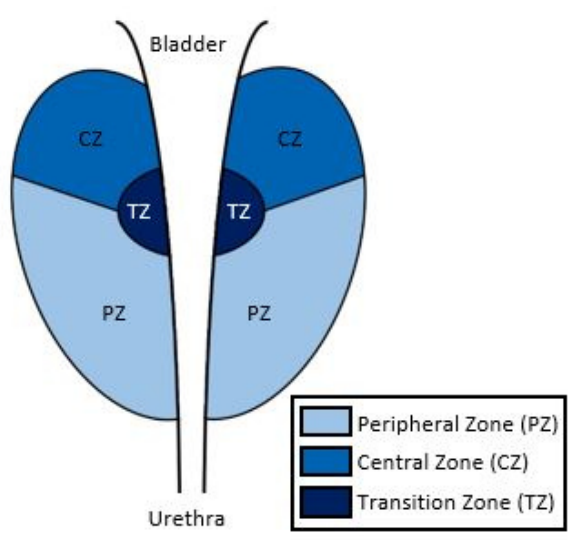

Figure 1. Diagram of the three prostate zones.

Mainly, the prostate gland has two functions. First, it produces seminal fluid that is injected into the urethra along with sperm when a male is sexually aroused [6]. Second, it controls the diameter of the urethra, thereby controlling the flow of urine [6]. To accomplish these functions, the prostate contains three main cell types: (1) gland cells that excrete seminal fluid, (2) muscles cells that control the diameter of the urethra for urine flow and ejaculation, and (3) fibrous cells that make up the supportive structure of the prostate [6].

In pathology, the prostate is divided into three different regions (zones): the central zone (CZ), the transition zone (TZ), and the peripheral zone (PZ) [8-10]. Fig. 1 illustrates the anatomy of the prostate and its glandular zones. The CZ, TZ, and $\mathrm{PZ}$ constitute $25 \%, 5 \%$, and $70 \%$ of the prostate, respectively [8]. Each of these zones consists of different cell types and consequently have different cancer occurrence rates. The PZ is mainly derived from the urogenital sinus and the $\mathrm{TZ}$ is derived from similar cell types. The $\mathrm{CZ}$, however, is derived from the Wolffian duct [11]. The vast majority (70\%) of cancerous prostate tumors develop in the PZ, while only $25 \%$ occur in the TZ and 5\% in the CZ [11]. This makes sense because the PZ and TZ have similar embryological origins. To detect and diagnose the cancerous cells in the prostate, several diagnosis techniques can be employed. The methods reviewed can be categorized as in-vitro techniques and in-vivo tech- 
niques. In, Section A, the basic in-vitro techniques for prostate cancer diagnosis. Section B details the current in-vivo CAD systems for prostate cancer using the different types of medical image modalities are outlined. Discussion of the work in this review is presented in section $C$ and the challenges that faced by current CAD systems for prostate cancer and the recent trends to solve these challenges are highlighted. Finally, section D concludes the work done in this review.

\section{A In-Vitro Prostate Cancer Diagnostic Technologies}

In the literature, several methods and techniques have been investigated to provide tools for prostate cancer. These tools include one or more types of invitro diagnostic techniques, which involve collecting a physical sample (i.e., blood, urine, or tissue) from the patient. Before a physical sample is taken from a patient, a digital rectal exam (DRE) is often performed. This consists of a skilled physician manually feeling for any abnormalities in the prostate gland through the rectum. The DRE examination is inexpensive and easy to perform. However, the accuracy of a DRE examination is not high and depends on the physician's experience. Also, it can only detect sufficiently large tumors. For detecting smaller tumors, in-vitro and/or in-vivo tests should be conducted [12]. The two major categories of invitro techniques are biomarker tests needle biopsies $[12,13]$. In this section, a brief overview of in-vitro cancer diagnostic tools and related research studies conducted in the past decade will be given.

Biomarker tests are common methods for detecting prostate cancer in a pa-

tient. These tests can be categorized as blood-based tests, urine-based tests, and hybrid tests (see Fig. 2). Each of these tests have different accuracies and applications.

Blood-based tests are the most common biomarker examinations for diagnosis. These methods require drawing blood from a patient, and are therefore classified as invasive techniques. The main type of blood-based biomarker used in the 


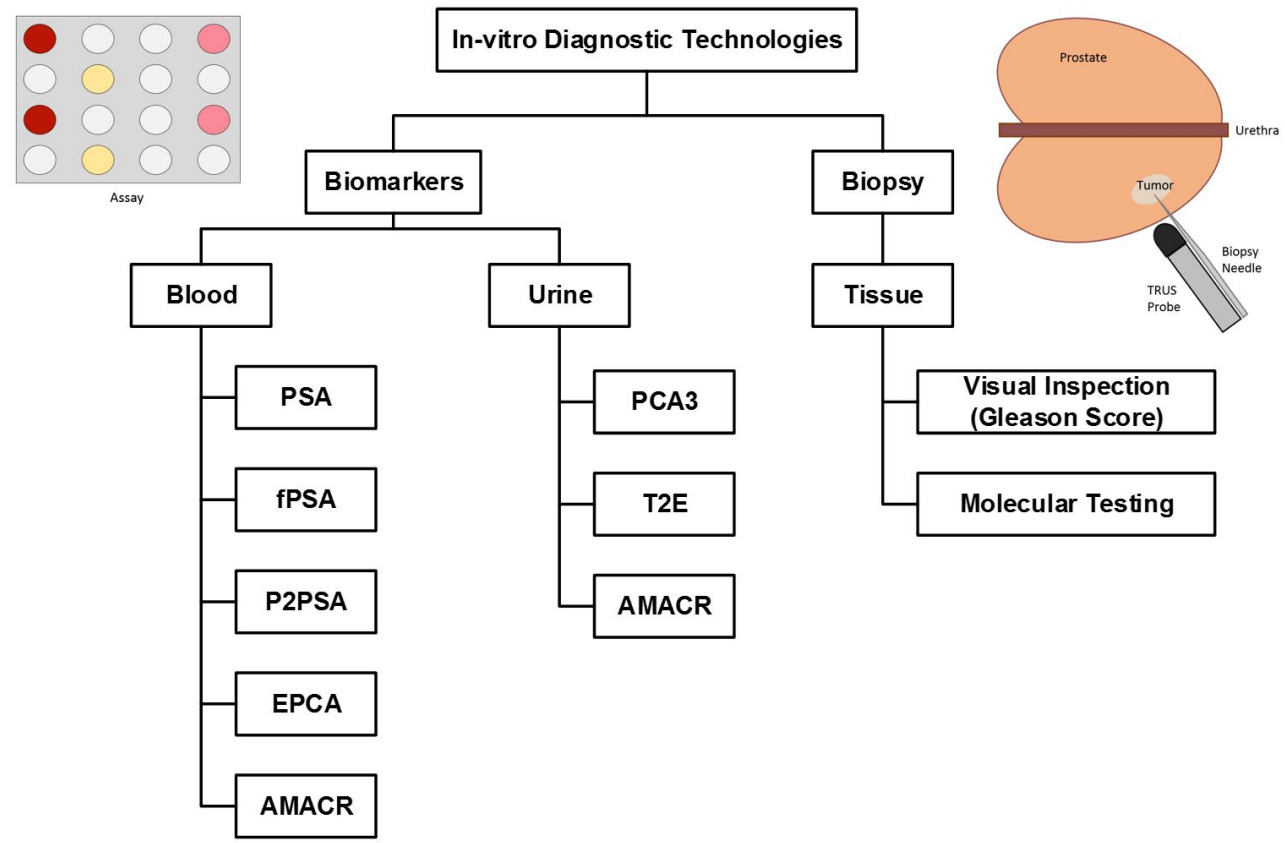

Figure 2. Different in-vitro biomarkers that are clinically used for prostate cancer diagnosis.

literature and in a clinical setting is PSA, which is a serine protease produced by correctly functioning and cancerous prostates [14]. Malignant prostates expel significantly more PSA into the human circulatory system [14]. This increased level of PSA in the blood can then be used to indicate a cancerous prostate. Several studies [15-24] evaluated the effectiveness of testing the overall amount of PSA for detecting whether a patient currently suffers from prostate cancer. These studies [15-24] reported different diagnostic accuracies, where the AUC ranged from 0.492-0.72. A PSA study by Sreekumar et al. [15] showed an AUC of 0.492 for 126 subjects. A 2,034 patient study was performed by Le et al. [16], which showed an AUC of 0.50. Additionally, Marks et al. [25] showed PSA diagnosis had an AUC of 0.524 for 233 subjects. Similarly, Catalona et al. [17] found PSA diagnosis to have 
an AUC of 0.525 for 1,372 subjects. Chun et al. [18] conducted an 809 patient study that showed an AUC of 0.53 for PSA. Another study by Deras et al. [19] had an AUC of 0.55 for 570 subjects. An additional study by van Gils et al. [20] concluded that PSA had an AUC of 0.57 for 583 subjects. Roobol et al. [21] found PSA diagnosis to have an AUC of 0.58 for 721 patients. Haese et al. [22] conducted a 463 subject study that showed an AUC of 0.60 for PSA. Another study by Ankerst et al. [23] found PSA to have an AUC of 0.607 for 443 patients. In a study conducted by Salami et al. [24], PSA had an AUC of 0.72 for 45 subjects. Other applications of PSA include the prediction of future advanced prostate cancer. study conducted by Ulmert et al. considered predicted cancer up to 25 years after the test as a successful diagnosis and had an AUC of 0.791 for 21,277 subjects [26]. However, this could be useful in long term care, but not necessarily in diagnosing whether or not a patient currently has prostate cancer. The main limitation of PSA-based diagnosis is its association with a high-risk of over diagnosis of prostate cancer as higher PSA levels may reflect other conditions, such as an enlarged or inflamed prostate [27].

In addition to the overall PSA in a blood sample, the amount of several specific types of PSA in a sample have also been used for diagnosing prostate cancer. Two common types of PSA used in prostate cancer diagnosis are the free prostate specific antigen (fPSA), PSA not bound to serum proteins, and the [-2] isoform of proenzyme prostate specific antigen (p2PSA). Additionally, the ratio of fPSA to PSA (\%fPSA) and the ratio of p2PSA to PSA (\%p2PSA) are common PSA measures for diagnosing prostate cancer. A 2,034 subject study by Le et al. [16] found that \%fPSA-based diagnosis had an AUC of $0.68, \%$ p2PSA-based diagnosis had an AUC of 0.76, and the Beckman Coulter prostate health index (PHI), a combined measurement of fPSA and p2PSA, had an AUC of 0.77. A similar study was performed by Catalona et al. [17], which found that diagnosing using \%fPSA had an AUC of 0.525, using fPSA had an AUC of 0.615, using p2PSA had an AUC of 0.557, 
and using PHI had an AUC of 0.703 for 1,372 subjects. Additionally, Ferro et al. [28] found that fPSA had an AUC of 0.60, \%fPSA had an AUC of 0.62, \%p2PSA had an AUC of 0.76, and PHI had an AUC of 0.77 for 300 subjects.

Other blood-based biomarkers have been researched for diagnosing prostate cancer, such as the early prostate cancer antigen (EPCA) [29,30]. EPCA is a nuclear matrix protein that showed a promising results for diagnosing prostate cancer [31,32]. For example, Paul et al. [33] developed an EPCA assay technique and testing using 46 subjects demonstrated a sensitivity of $92 \%$ and a specificity of $94 \%$ for the technique. In addition, $\alpha$-methylacyl-CoA racemase (AMACR) is yet another researched biomarker [30]. It can be used in a blood-based, a urine-based, or a tissue-based (after biopsy) test for diagnosing prostate cancer [29]. AMACR is an enzyme utilized in the synthesis and the oxidative metabolism of branched fatty acids [30]. A reduced level of AMACR has been linked to prostate cancer [34]. Lin et al. [35] developed and tested a new blood-based nanoparticle electrochemical AMACR biosensor assay. This device was shown to have an accuracy of $100 \%$ for 24 subjects. However, due to the limited number of test subjects, their developed device needs further investigation.

Urine-based biomarker tests have been investigated as a non-invasive method to indicate prostate cancer. A common urine-based biomarker is PCA3 (formerly DD3), a prostate specific non-coding RNA [29, 30, 32,36]. The reported studies [20, 21, 25, 28, 37-39] showed an AUC ranging from 0.64-0.74 for PCA3. Hessel et al. [37] studied the effectiveness of this biomarker in diagnosing prostate cancer. This 108 subject study showed a sensitivity of $67 \%$, a specificity of $83 \%$, and an AUC of 0.717. A study performed by Marks et al. [25] showed that a PCA3-based assay test had a sensitivity of $58 \%$, a specificity of $72 \%$, and an AUC of 0.68 for 233 subjects. Another PCA3 study conducted by van Gils et al. [20] tested a fluorescence-based PCA3 technique using 583 subjects. It had a sensitivity of $65 \%$, a specificity of $66 \%$, and an AUC of 0.66. Roobol et al. [21] also tested PSA and PCA3 biomarkers for 
721 subjects and found an AUC of 0.58 for PSA-based tests and an AUC of 0.64 for PCA3-based tests. In addition, Ferro et al. [28] performed a PCA3 study that resulted in an AUC of 0.73 for 300 subjects.

Other urine-based biomarkers have been researched for diagnosing prostate cancer, which include AMACR (which also can be performed using blood and tissue samples) and the gene fusion of the serine 2 and E-twenty-six related genes (ERGs) known as TMPRSS2-ERG or T2E. Since a reduced level of AMACR has been linked to prostate cancer [34], a study by Sreekumar et al. [15] used AMACR in a urine-based assay technique and achieved a sensitivity of $77.8 \%$, a specificity of $80.6 \%$, and an AUC of 0.789 for 128 subjects. Other studies [24,30,40,41] used TMPRSS2-ERG for cancer detection, since it becomes rearranged in approximately $80 \%$ of prostate cancer cases $[12,30,42]$. These studies reported AUC values ranging from 0.63-0.88 for cancer diagnosis. A study by Stephan et al. [41] compared TMPRSS2-ERG, PCA3, and PHI and found that they had an AUC of 0.63, 0.74, and 0.68 , respectively. However, there were no statistical differences between PCA3 and PHI for the 110 subjects tested.

Hybrid-based tests investigate the integration of both blood and urine tests to increase the accuracy of diagnosis. For example, TMPRSS2-ERG has been used in conjunction with PCA3 and PSA to diagnose prostate cancer from urine samples [30]. Salami et al. [24] compared the effectiveness of TMPRSS2ERG, PCA3 and the combination of PSA, PCA3, and TMPRSS2-ERG for prostate cancer diagnosis on 45 subjects. TMPRSS2-ERG alone had a sensitivity of $67 \%$, a specificity of $87 \%$, and an AUC of 0.77 . PCA3 alone had a sensitivity of $93 \%$, a specificity of $37 \%$, and an AUC of 0.65 . Finally, the combined test had a sensitivity of $90 \%$, a specificity of $80 \%$, and an AUC of 0.88 . Also, Leyten et al. [40] developed a multivariate regression model using PCA3, TMPRSS2ERG, PSA, DRE, PV, and the outcome of manual Transrectal Ultrasound (TRUS) analysis, which had an AUC of 0.842 for 443 patients. Additionally, Lin et al. [43] tested TMPRSS2-ERG, PCA3, and the 
combination of PSA, PCA3, and TMPRSS2-ERG for prostate cancer diagnosis on 387 subjects. This resulted in AUCs of 0.66, 0.66, and 0.70, respectively.

In addition, hybrid analysis of PSA (from a blood sample) and PCA3 (from a urine sample) has been investigated to increase the diagnostic accuracy. Crawford et al. [38] tested PSA, PCA3, and their sequential combination for diagnosing prostate cancer. The results were AUCs of $0.569,0.706$, and 0.720 for 1,913 subjects, respectively. Groskopf et al. [39] investigated the PCA3 to PSA ratio as a diagnostic measure and found that it had an AUC of 0.746 for 143 patients. Also, Ankerst et al. [23] tested a Bayesian probability model built using PSA, PCA, DRE, and family history data for diagnosing prostate cancer. However, this technique had an AUC of 0.696 , which was not significantly more accurate than the AUC of PCA alone, 0.653 , for the 443 subjects used in the study.

Multi-variable regression models have also been developed for prostate cancer diagnosis based on combing values of PSA and PCA3 with other diagnostic features. These models had an AUC ranging from 0.45-0.83. For example, Deras et al. [19] performed assay-based experiments that achieved an AUC of 0.55 for PSA, an AUC of 0.69 for PCA, and an AUC of 0.75 for a logistic regression technique [44] that utilized PSA, PCA3, prostate volume (PV), and DRE results for 570 subjects. Chun et al. [18] developed another logistic regression model that utilized PSA and PCA3 assay data. Testing using 809 subjects showed an AUC of 0.53 for PSA, an AUC of 0.68 for PCA3, and an AUC of 0.73 for a logistic regression model based on PSA, PCA3, PV, DRE, the patient's age, and the patient's biopsy history $(\mathrm{Bx}-\mathrm{H})$. Additionally, Haese et al. [22] proposed another logistic regression model based technique that used biomarker assays. This 463 subject study showed an accuracy of 0.60 for PSA, an accuracy of 0.58 for \%fPSA, an accuracy of 0.66 for PCA3, and an accuracy of 0.71 for a logistic regression model based method that used PSA, \%fPSA, PCA3, PV, DRE, and patient age for 463 subjects. Also, a PCA3 assay was developed by Auprich et al. in [45] based on the study performed in [39]. This 
method created a logistic regression model that utilized PCA3 data acquired using this assay as well as PSA, PV, DRE, age and Bx-H data. This model had an AUC of 0.75 for 621 patients. An additional logistic regression model that used PSA, \%fPSA, PCA3, PV, DRE, Bx-H, family history, patient age, number of biopsy cores, and clinical analysis if TRUS images was developed and tested by Perdona et al. [46]. This method had a sensitivity of $70 \%$, a specificity of $81 \%$, and an AUC of 0.83 for 218 subjects. Hansen et al. [47] analyzed another regression model that used PSA, PCA3, DRE, and PV data. This technique had a sensitivity of $79 \%$, a specificity of $59 \%$, and an AUC of 0.69 for 692 subjects. PSA and PCA3 biomarkers have been used in several studies to determine whether a patient has a tumor with a volume greater than $0.5 \mathrm{ml}$. Nakanishi et al. [48] employed these biomarkers for 142 subjects and achieved AUCs of 0.63 for PSA and 0.76 for PCA3 for diagnosing tumors with a volume greater than $0.5 \mathrm{ml}$. Auprich et al. [49] created a logistic regression model based on PSA, PPC, PCA3, and biopsy Gleason score data and reported an AUC of 0.84 for diagnosing tumors with a volume greater than $0.5 \mathrm{ml}$ for 160 patient. Table 1 summarizes the in-vitro studies that investigate the use of fluid-borne biomarkers for diagnosing prostate cancer, including the biomarkers used in each study, the number of test subjects, and the reported performance.

Needle biopsies usually follow a DRE or biomarker analysis, commonly a PSA blood test, in order to collect a tissue sample for cancer diagnosis. This is due to the fact that there is a high potential for the current clinical biomarker tests to classify incorrectly [50,51]. A TRUS guided prostate biopsy is the standard technique for collecting these tissue samples [52]. Once they have been acquired, tissue analysis is conducted to diagnose the prostate tumors either visually or by analyzing tissue-based extracted biomarkers. The most common method to analyze the tissue sample is the Gleason grading system [53], which is performed visually by a physician. The Gleason score was developed by Gleason and Mellinger in 1974 [54]. This measure is based on the two most prevalent cancer patterns in 
the collected tissue sample. A physician grades each pattern on a scale from 1-5, going from non-cancerous to highly cancerous, via visual analysis. The Gleason score is then calculated by summing these two values. If only one cancer pattern is present, the Gleason score is twice the individual pattern score. A score greater than or equal to six is seen as a strong indicator of cancer [53]. Even though this method is widely used, it is not a completely quantitative technique and different observers may classify a sample differently, leading to discrepancies in the diagnosis [55]. In addition to the visually-assessed Gleason scores, other tissue tests has been performed based on analyzing specific biomarkers in the sample tissue. For example, Jiang et al. [56] proposed a method that used the real-time polymerase chain reaction technique [57] to test tissue samples for AMACR. This method had a sensitivity of $97 \%$ and a specificity of $92 \%$ for 807 subjects [56].

In summary, DRE, biomarker analysis, and needle biopsies are common diagnostic techniques for prostate cancer. However, they have several disadvantages. DRE is highly invasive and is subject to a physicians subjective analysis. Also, biomarker tests can have high false positive and false negative rates [50,51]. This can lead to patients in need of treatment not receiving it while patients without prostate cancer are treated. Additionally, these tests require a physical sample, wether it be blood or urine [29]. This is also true for needle biopsies, which are highly invasive and can cause physical harm to patients (e.g., bleeding). Gleason scores require biopsies to invasively collect tissue samples and are dependent on the observer analyzing the sample [53]. However, biopsies remain the gold standard for diagnosis of prostate cancer, but are the last resort because of their invasive nature, high cost, and potential morbidity rate. Additionally, the relatively small needle biopsy samples have a higher possibility of producing false positive diagnosis. A non-invasive and quantitative method for diagnosing prostate cancer would eliminate the need for collecting physical patient samples and increase the overall accuracy of diagnosis. 
TABLE 1: Summary of in-vitro studies for diagnosing prostate cancer based on fluid-borne biomarkers, including the biomarkers used in each study, the number of test subjects, and the reported performance.

\begin{tabular}{|c|c|c|c|}
\hline Study & Data & Biomarkers & Performance \\
\hline Paul et al. [33] & $\begin{array}{l}46 \text { Subjects ( } 34 \\
\text { Control and } 12 \\
\text { Cancerous) }\end{array}$ & - EPCA & $\begin{array}{l}\text { - SEN: } 0.92 \\
\text { - SPE: } 0.94\end{array}$ \\
\hline $\begin{array}{l}\text { Sreekumar et al. } \\
\text { [15] }\end{array}$ & $\begin{array}{l}126 \text { Subjects } \\
(36 \quad \text { Control } \\
\text { and } 90 \text { Can- } \\
\text { cerous) }\end{array}$ & $\begin{array}{l}\text { - PSA } \\
\text { - AMACR }\end{array}$ & $\begin{array}{l}\text { - SEN (PSA): } \\
0.456 \\
\text { - SPE (PSA): } \\
0.50 \\
\text { - AUC (PSA): } \\
0.492 \\
\text { - SEN } \\
\text { (AMACR): } \\
0.778 \\
\text { - SPE } \\
\text { (AMACR): } \\
0.806 \\
\text { - AUC } \\
\text { (AMACR): } \\
0.789\end{array}$ \\
\hline Jiang et al. [56] & 807 Subjects & - $\mathrm{AMACR}$ & $\begin{array}{l}\text { - SEN: } 0.97 \\
\text { - SPE: } 0.92\end{array}$ \\
\hline
\end{tabular}


TABLE 1 - continued from previous page

\begin{tabular}{|c|c|c|c|}
\hline Study & Data & Biomarkers & Performance \\
\hline Le et al. [16] & 2,034 Subjects & $\begin{array}{l}\text { - PSA } \\
\text { - } \% \text { fPSA } \\
\text { - } \% \text { p2PSA } \\
\text { - } \mathrm{PHI}\end{array}$ & $\begin{array}{l}\text { - AUC (PSA): } \\
0.50 \\
\text { - AUC } \\
(\% \text { fPSA): } \\
0.68 \\
\text { - AUC } \\
\text { (\%p2PSA): } \\
0.76 \\
\text { AUC (PHI): } \\
0.77\end{array}$ \\
\hline $\begin{array}{l}\text { Catalona et } \\
\text { al. [17] }\end{array}$ & 1,372 Subjects & $\begin{array}{l}\text { - PSA } \\
\text { - } \mathrm{fPSA} \\
\text { - p2PSA } \\
\text { - PHI }\end{array}$ & $\begin{array}{l}\text { - AUC (PSA): } \\
0.525 \\
\text { - AUC (fPSA): } \\
0.615 \\
\text { - AUC } \\
\text { (\%fPSA): } \\
0.648 \\
\text { - AUC (p2PSA): } \\
0.557 \\
\text { - AUC (PHI): } \\
0.703\end{array}$ \\
\hline
\end{tabular}


TABLE 1 - continued from previous page

\begin{tabular}{|c|c|c|c|}
\hline Study & Data & Biomarkers & Performance \\
\hline Hessel et al. [37] & 108 Subjects & - PCA3 & $\begin{array}{l}\text { - SEN: } 0.67 \\
\text { - SPE: } 0.83 \\
\text { - AUC: } 0.717\end{array}$ \\
\hline $\begin{array}{l}\text { Groskopf et al. } \\
{[39]}\end{array}$ & 143 Subjects & $\begin{array}{l}\text { - } \mathrm{PSA} \\
\text { - } \mathrm{PCA} 3\end{array}$ & $\begin{aligned} \text { - } & \text { SEN } \\
& (\mathrm{PCA} 3 / \mathrm{PSA}): \\
& 0.69 \\
\text { - } & \text { SPE } \\
& (\mathrm{PCA} 3 / \mathrm{PSA}): \\
& 0.79 \\
\text { - } & \text { AUC } \\
& (\mathrm{PCA} 3 / \mathrm{PSA}): \\
& 0.746\end{aligned}$ \\
\hline Marks et al. [25] & 233 Subjects & $\begin{array}{l}\text { - PSA } \\
\text { - PCA3 }\end{array}$ & $\begin{array}{l}\text { - AUC (PSA): } \\
0.524 \\
\text { - SEN (PCA3): } \\
0.58 \\
\text { - SPE (PCA3): } \\
0.72 \\
\text { - AUC } \\
\text { (PCA3):0.68 }\end{array}$ \\
\hline
\end{tabular}


TABLE 1 - continued from previous page

\begin{tabular}{|c|c|c|c|}
\hline Study & Data & Biomarkers & Performance \\
\hline Ferro et al. [28] & 300 Subjects & $\begin{array}{l}\text { - } \mathrm{fPSA} \\
\text { - } \% \mathrm{fPSA} \\
\text { - } \mathrm{p} 2 \mathrm{PSA} \\
\text { - PHI } \\
\text { - PCA }\end{array}$ & $\begin{array}{l}\text { - AUC (fPSA): } \\
0.60 \\
\text { - } \text { AUC } \\
\text { (\%fPSA):0.62 } \\
\text { - AUC (p2PSA): } \\
0.63 \\
\text { - AUC (PHI): } \\
0.77 \\
\text { - AUC (PCA): } \\
0.73\end{array}$ \\
\hline van Gils et al. [20] & 583 Subjects & $\begin{array}{l}\text { - PSA } \\
\text { - PCA3 }\end{array}$ & $\begin{array}{ll}\text { - AUC } & \text { (PSA): } \\
0.57 & \\
\text { - SEN (PCA3): } \\
0.65 & \\
\text { - SPE (PCA3): } \\
0.66 \\
\text { - AUC (PCA3): } \\
0.66\end{array}$ \\
\hline
\end{tabular}


TABLE 1 - continued from previous page

\begin{tabular}{|c|c|c|c|}
\hline Study & Data & Biomarkers & Performance \\
\hline Haese et al. [22] & 463 Subjects & $\begin{array}{l}\text { - PSA } \\
\text { - } \% f \text { PSA } \\
\text { - PCA3 }\end{array}$ & $\begin{array}{l}\text { - ACC (PSA): } \\
0.60 \\
\text { - ACC (\%fPSA): } \\
0.58 \\
\text { - ACC(PCA): } \\
0.66 \\
\text { - ACC (PSA, } \\
\text { \%fPSA, PCA3, } \\
\text { PV, DRE, } \\
\text { Age): } 0.71\end{array}$ \\
\hline Ankerst et al. [23] & 443 Subjects & $\begin{array}{l}\text { - PSA } \\
\text { - PCA3 }\end{array}$ & $\begin{array}{l}\text { - AUC (PSA): } \\
0.607 \\
\text { - AUC (PCA): } \\
0.665 \\
\text { - AUC (PSA, } \\
\text { PCA3, DRE, } \\
\text { FH): } 0.696\end{array}$ \\
\hline Deras et al. [19] & 570 Subjects & $\begin{array}{l}\text { - PSA } \\
\text { - PCA3 }\end{array}$ & $\begin{array}{l}\text { - AUC (PSA): } \\
0.55 \\
\text { - AUC (PCA3): } \\
0.69 \\
\text { - AUC (PSA, } \\
\text { PCA3, PV, } \\
\text { DRE): } 0.75\end{array}$ \\
\hline
\end{tabular}


TABLE 1 - continued from previous page

\begin{tabular}{|c|c|c|c|}
\hline Study & Data & Biomarkers & Performance \\
\hline Chun et al. [18] & 809 Subjects & $\begin{array}{l}\text { - PSA } \\
\text { - PCA3 }\end{array}$ & $\begin{array}{l}\text { - AUC (PSA): } \\
0.53 \\
\text { - AUC (PCA3): } \\
0.68 \\
\text { - AUC (PSA, } \\
\text { PCA3, PV, } \\
\text { DRE, Age, } \\
\text { Bx-H): } 0.73\end{array}$ \\
\hline Roobol et al. [21] & 721 Subjects & $\begin{array}{l}\text { - PSA } \\
\text { - } \mathrm{PCA} 3\end{array}$ & $\begin{array}{l}\text { - } \text { AUC (PSA): } \\
0.58 \\
\text { - } \text { AUC } \\
\text { (PCA3): } 0.64\end{array}$ \\
\hline Auprich et al. [45] & 621 Subjects & $\begin{array}{l}\text { - PSA } \\
\text { - PCA3 }\end{array}$ & $\begin{array}{ll}\text { - } & \text { AUC } \\
\text { PCA3, } & \text { PV, } \\
\text { DRE, Age, } \\
\text { Bx-H): } 0.75\end{array}$ \\
\hline
\end{tabular}


TABLE 1 - continued from previous page

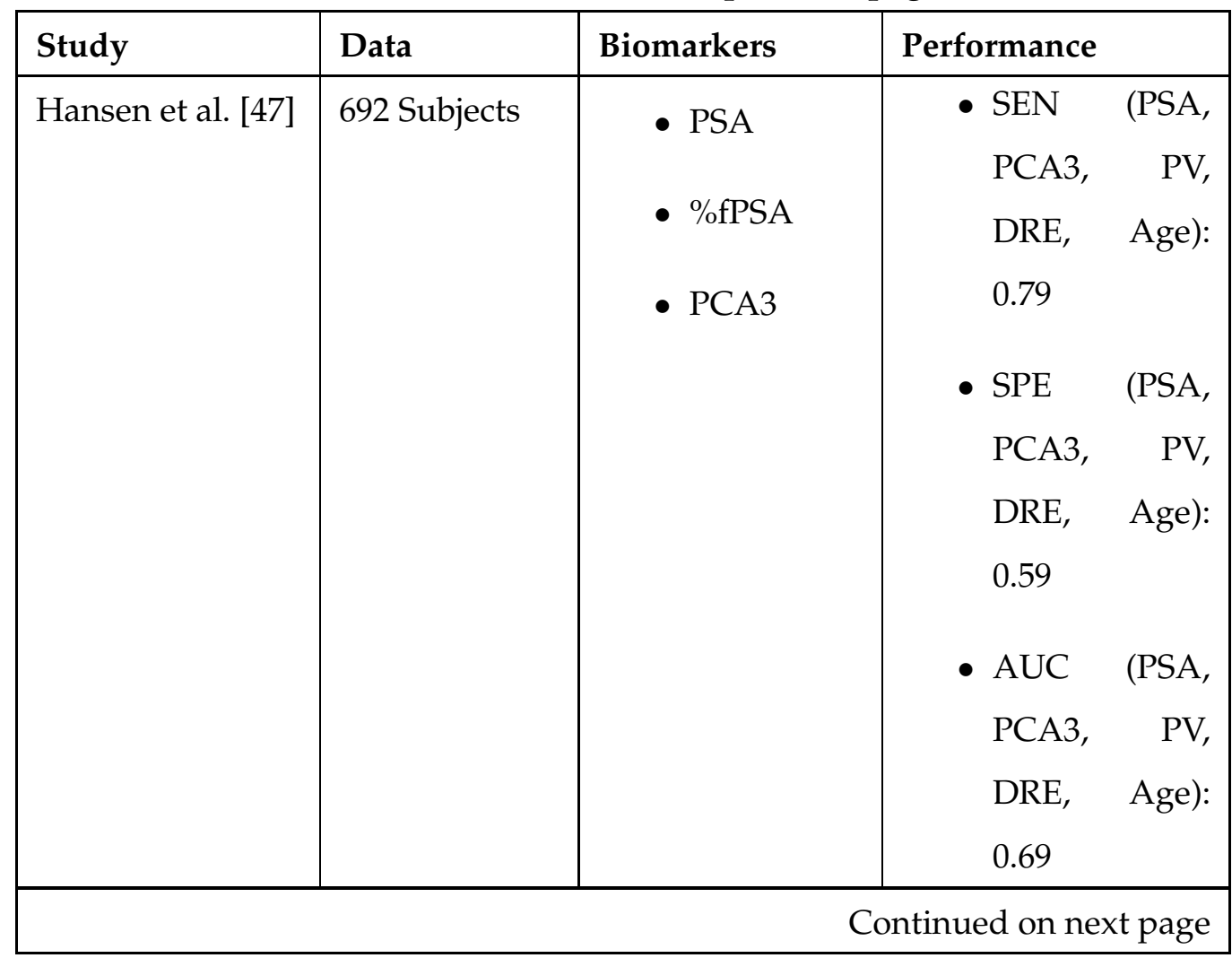


TABLE 1 - continued from previous page

\begin{tabular}{|c|c|c|c|}
\hline Study & Data & Biomarkers & Performance \\
\hline Perdona et al. [46] & 218 Subjects & $\begin{array}{l}\text { - PSA } \\
\text { - } \% f P S A \\
\text { - PCA3 }\end{array}$ & 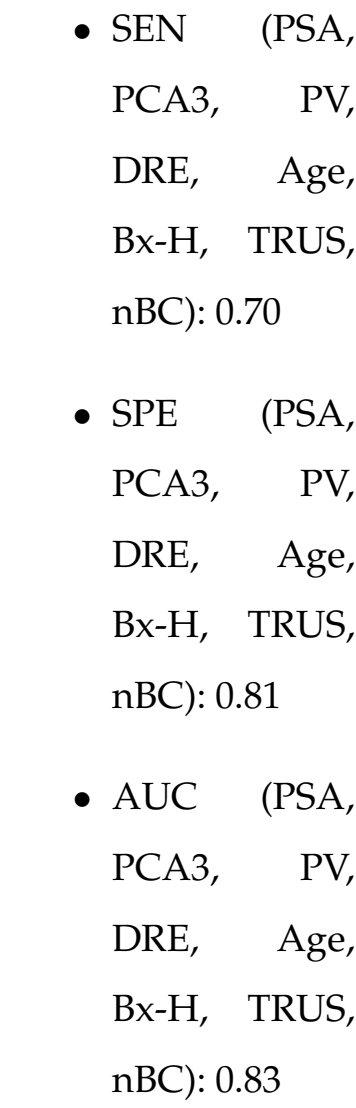 \\
\hline $\begin{array}{l}\text { Crawford et al. } \\
{[38]}\end{array}$ & 1913 Subjects & $\begin{array}{l}\text { - PSA } \\
\text { - PCA3 }\end{array}$ & $\begin{array}{l}\text { - AUC (PSA): } \\
0.569 \\
\text { - AUC (PCA3): } \\
0.706 \\
\text { - AUC (PSA, } \\
\text { PCA3): } 0.720\end{array}$ \\
\hline
\end{tabular}


TABLE 1 - continued from previous page

\begin{tabular}{|c|c|c|c|}
\hline Study & Data & Biomarkers & Performance \\
\hline Salami et al. [24] & 45 Subjects & $\begin{array}{l}\text { - } \mathrm{PSA} \\
\text { - } \mathrm{PCA} 3 \\
\text { - } \mathrm{T} 2 \mathrm{E}\end{array}$ &  \\
\hline
\end{tabular}


TABLE 1 - continued from previous page

\begin{tabular}{|c|c|c|c|}
\hline Study & Data & Biomarkers & Performance \\
\hline Leyten et al. [40] & 443 Subjects & $\begin{array}{l}\text { - } \mathrm{PSA} \\
\text { - } \mathrm{PCA} 3 \\
\text { - } \mathrm{T} 2 \mathrm{E}\end{array}$ & $\begin{array}{cc}\text { - AUC } & \text { (PCA3, } \\
\text { T2E, } & \text { PSA, } \\
\text { DRE, } & \text { PV, } \\
\text { TRUS): } & 0.842\end{array}$ \\
\hline Lin et al. [35] & 24 Subjects & - $\mathrm{AMACR}$ & - ACC: 1.0 \\
\hline Lin et al. [43] & 387 Subjects & $\begin{array}{l}\text { - } \mathrm{PSA} \\
\text { - } \mathrm{PCA} 3 \\
\text { - } \mathrm{T} 2 \mathrm{E}\end{array}$ & 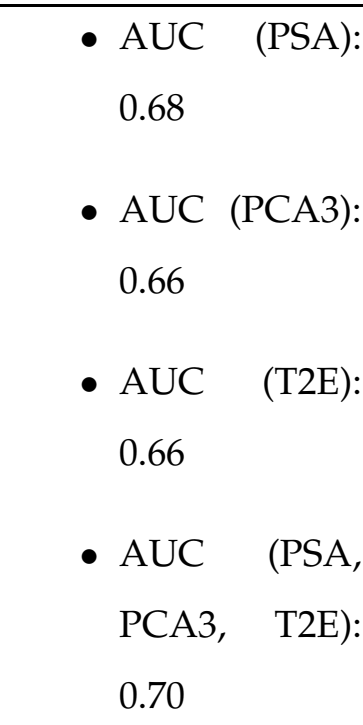 \\
\hline Stephan et al. [41] & 110 Subjects & $\begin{array}{l}\text { - } \mathrm{PHI} \\
\text { - } \mathrm{PCA} 3 \\
\text { - } \mathrm{T} 2 \mathrm{E}\end{array}$ & 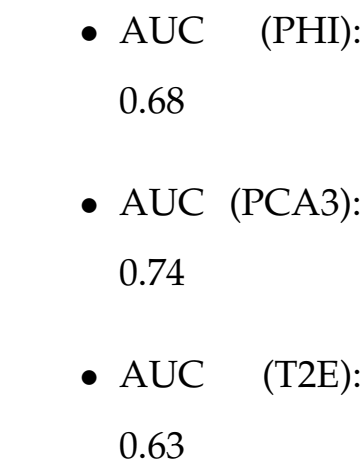 \\
\hline \multicolumn{4}{|c|}{$\begin{array}{l}\text { *ACC denotes accuracy. } \\
\text { *ROC denotes receiver operating characteristic. } \\
\text { *AUC denotes area under the ROC curve. } \\
\text { *PPV denotes positive predictive value. }\end{array}$} \\
\hline
\end{tabular}


TABLE 1 - continued from previous page

\begin{tabular}{|c|c|c|c|}
\hline Study & Data & Biomarkers & Performance \\
\hline \multicolumn{4}{|c|}{ *SEN denotes sensitivity. } \\
\hline \multicolumn{4}{|c|}{ *SPE denotes specificity. } \\
\hline \multicolumn{4}{|c|}{ *DRE denotes digital rectal exam. } \\
\hline \multicolumn{4}{|c|}{ *PV denotes prostate volume. } \\
\hline \multicolumn{4}{|c|}{${ }^{*} \mathrm{Bx}-\mathrm{H}$ denotes biopsy history. } \\
\hline \multicolumn{4}{|c|}{${ }^{*} \mathrm{FH}$ denotes family history. } \\
\hline \multicolumn{4}{|c|}{ *nBC denotes number of biopsy cores. } \\
\hline *T2E d & RSS2-E & & \\
\hline
\end{tabular}

\section{B In-vivo Prostate Cancer Diagnostic Techniques}

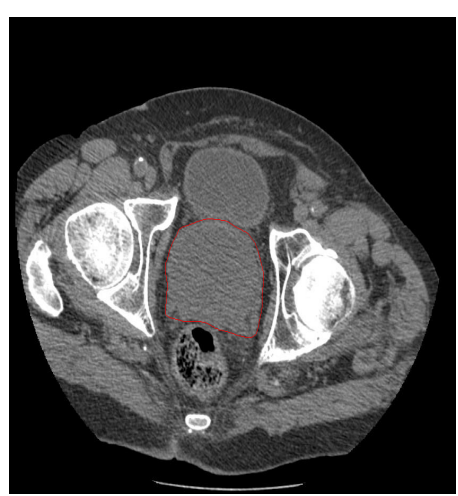

(a)

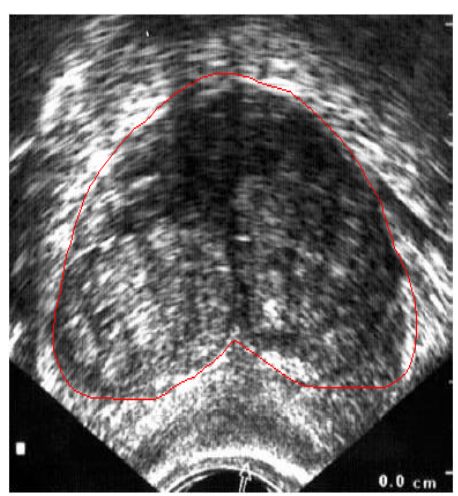

(b)

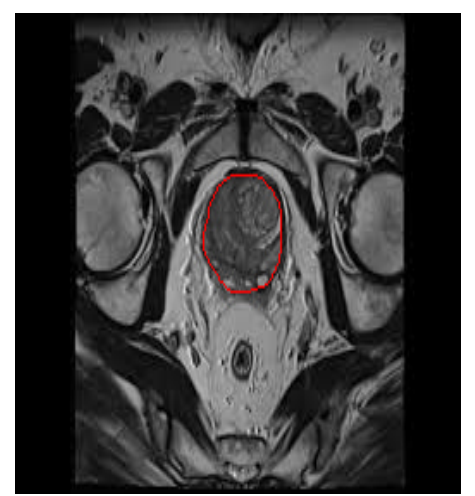

(c)

Figure 3. Typical CT (a), TRUS (b) and T2-MR (c) images of a prostate.

Recently, in-vivo image-based techniques have demonstrated the provenability to detect prostate cancer without the associated deleterious side effects of invasive techniques. These noninvasive methods for prostate cancer diagnosis are based on acquiring scans of the prostate and analyzing these scans for cancer detection. To acquire scans of the prostate, different medical imaging techniques, such as TRUS, magnetic resonance imaging (MRI) and computed tomography (CT), 
have been used (see Fig. 3). Each of these image modalities has its own mechanism for providing relevant physiological information of the prostate as well as its own advantages and limitations. For example, CT is currently used for post-therapy evaluation by physicians to assess the effectiveness of treatment [58]. However, it is expensive, uses radiation, and has poor contrast between soft tissues [58]. As a result, TRUS and MRI are more commonly used in CAD systems for diagnosing prostate cancer.

TRUS is the most frequently used technique for prostate imaging [58]. It is often used in planning and guiding needle biopsies [59]. In addition, TRUS is used in estimating the volume of the prostate gland, which can be used in biomarker screening [58]. TRUS is often chosen because it is relatively inexpensive and allows for real-time imaging. However, it does have several disadvantages for use in CAD systems. TRUS images have low contrast and a low signal-to-noise (SNR) ratio [60]. As a result, it can be difficult to accurately detect tumors and locate cancerous cells using TRUS images.

MRI is another widely used imaging modality for detecting prostate cancer. The main advantage of MRI is that it offers the best soft tissue contrast compared to other image modalities, such as CT and TRUS [61]. However, MRI is sensitive to noise and image artifacts, has a relatively long and complex acquisition, and has a relatively high cost [58].

Several different MRI techniques have been extensively used in prostate cancer CAD systems. T1-weighted (T1-MRI) and T2-weighted (T2-MRI) are two basic MRI techniques that measure the spin-lattice (T1) and spin-spin (T2) relaxation times to create an image [62]. Although these MRI techniques provide excellent soft tissue contrast, they lack functional information. Therefore, these MR imaging techniques have limited ability to effectively locate and classify prostate cancer [63]. Dynamic contrast-enhanced MRI (DCE-MRI) is another MR technique based on using contrast agents to provide information about the anatomy, 




(a)

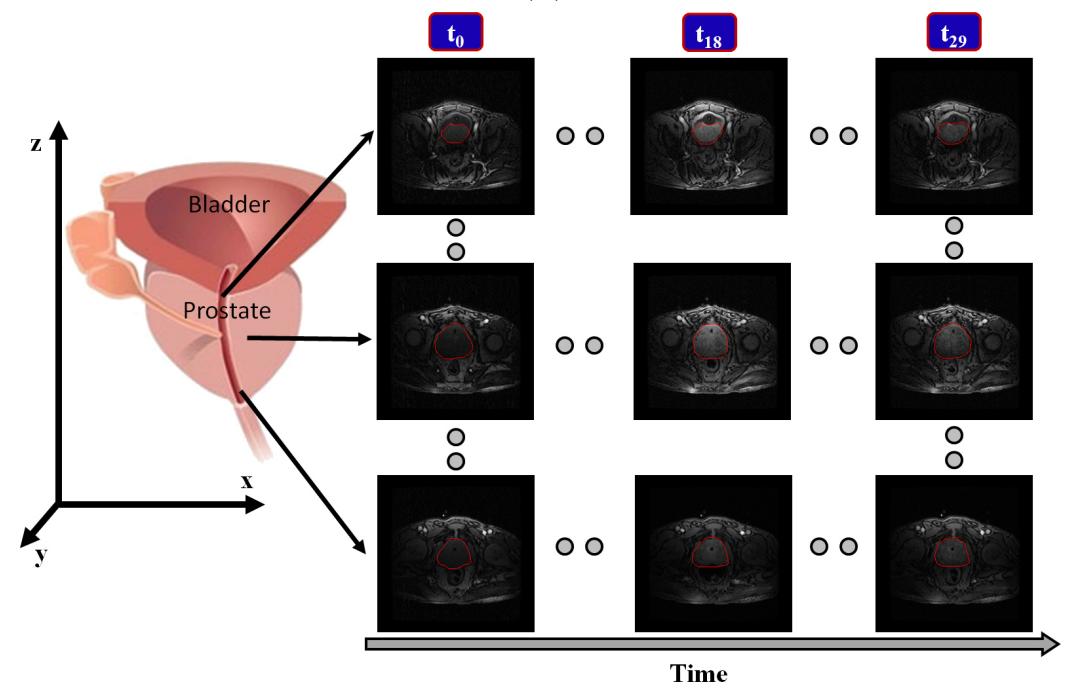

(b)

Figure 4. Different MR images of the prostate: (a) DWI-MRI, T2-MR (first column on the left in DWI-MRI; i.e., DWI-MRI at $b_{0}$ ) , and (b) DCE-MRI. Segmentation (red contour) is outlined by an expert.

function, and metabolism of target tissues [64]. In recent years, DCE-MRI has had considerable success in detecting and locating prostate cancer. However, intravenous administration of a contrast agent can potentially harm a patient's kid- 
neys [65]. In addition, injecting and waiting for the contrast agent to settle in the prostate increases the time required to scan the patient. Diffusion weighted imaging (DWI) [66] is an alternative MRI technique that avoids using contrast agents. DWI is a functional MRI technique that measures the micromovements (random, Brownian) of extracellular water molecules inside the body [67]. These movements provide indirect information about the structures surrounding these water molecules. Images collected using this modality have been shown to be useful for determining the size and shape of the prostate as well as detecting and locating cancerous tumors [66]. Typical MRI prostate images are shown in Fig. 4. In several CAD systems, a combination of these MRI techniques has been used for diagnosing prostate cancer [68-74]. This is often called multi-parametric imaging. These systems seek to extract different information from each type of image to detect, locate, and classify prostate tumors more accurately.

Development of CAD systems for detecting prostate cancer using these different image modalities is an ongoing area of research [75]. The success of CAD systems can be measured based on the diagnostic accuracy, speed, and automation level. Of the image modalities discussed, the most popular image modalities used for the diagnosis of prostate cancer are TRUS, T2-MRI, DCE-MRI, and DWI MRI. A typical CAD system for detecting prostate cancer, shown in Fig. 5, generally consists of three main processing steps: (1) prostate segmentation and/or tumor localization, (2) feature extraction, and (3) classification of the prostate tissue. The input to any CAD system is a set of medical images-a set of 2D time-series images or a series of 3D volumes- that contain the prostate. The first step in this system is the segmentation of the prostate or regions of interest (ROIs). This step can be performed automatically, semi-automatically, or manually by a radiologist. In the second step, a set of features (e.g., average grey level intensities) are extracted from the segmented prostate region and used to create a feature space. Finally, these features are used to classify prostate tissue as either benign or malignant us- 
ing one or more classifiers such as neural networks or support vector machines (SVM) [76]. The accuracy of these classification techniques is compared against a gold standard, usually a needle biopsy [77]. Below, several complete CAD systems for each image modality, their advantages, limitations, the computational techniques implemented in each system, as well as their reported experimental results are overviewed.



Figure 5. Diagram of a general fully-automated CAD system for prostate cancer.

\section{TRUS-based CAD systems}

Several fully automated CAD systems for prostate cancer detection have been proposed for TRUS. As illustrated in Fig 5, these systems take medical images as input, segment the prostate or ROIs, extract features from the selected image region, and then classify the selected region as cancerous or benign. In this section, the segmentation methods, the extracted features from TRUS images, and the current prostate cancer CAD systems developed in the last 10 years will be 
overviewed.

Prostate segmentation from in vivo TRUS images: Segmentation of the prostate from in vivo TRUS images is a very challenging problem for the following reasons: (1) TRUS images are often noisy, (2) the prostate boundary is not clearly defined, and (3) shadow artifacts can be present. Although manual outlining of the prostate border enables the prostate volume to be determined, it is time consuming and observer dependent. Therefore, different segmentation techniques have been proposed to address these challenges and accurately segment the prostate. The most popular techniques used for prostate segmentation are edge detection-based techniques, deformable model-based techniques, and statistical-based techniques.

Edge detection-based techniques use image information to find the pixels, or voxels in three dimensions (3D), that correspond to the edge of the prostate [78]. This method has been frequently used to segment the prostate from TRUS images. For example, Abolmaesumi and Sirouspour [79] proposed an automated technique to locate the prostate edges based on a probabilistic data estimator [80]. Also, Sahba et al. [81] proposed an automated technique that used morphological information [82], a Kalman estimator [83], and fuzzy inference to extract the edges of the prostate. The main limitation of edge detection techniques is that they do not work well with noisy images and/or objects with unclear or diffused edges.

Deformable models (DMs), developed by Kass et al. [84] and Caselles et al. [85], delineate an object's border in an image by evolving a deformable boundary towards the objects' edge based on image-derived features [86-93]. Various studies have employed different types of DMs for TRUS prostate segmentation, such as level set DMs [85,94], curve-fitted deformable boundaries, and active shape models (ASMs) [95]. Level set DM is a popular technique for medical image segmentation [96-99]. It has been widely used due to its flexible evolution and no need for parametrization [100]. For example, Wang et al. [101] proposed an automated technique for 2D prostate segmentation using a level set DM guided by 
the prior information of prostate shape and appearance. Level sets have also been used for 3D prostate segmentation, as can be seen by the method developed by Zhan et al. [102] that used a level set DM guided by prostate texture and shape information to perform 3D prostate segmentation.

Other DMs for prostate segmentation include curve-fitted deformable boundaries, e.g., fitting the prostate borders to an ellipse due to its approximation of the prostate's shape. For example, Gong et al. [103] used a semi-automated technique that represented a prostate's edge as a deformable super-ellipse that evolved to the prostate borders based on extracted region-based image features. Saroul et al. [104] proposed another technique which involve an appearance-guided DM and curve fitting to segment a prostate's border. Also, a semi-automated technique developed by Baidiei et al. [105] used an elliptical curve fitting to segment the prostate boundary. Additionally, Mahdavi et al. [106] proposed a semi-automated technique that applied ellipsoid curve fitting for segmenting the prostate from $3 \mathrm{D}$ TRUS images. However, ellipsoids have not been the only surface used for 3D prostate segmentation. Tutar et al. [107] proposed a DM for TRUS prostate segmentation that used spherical harmonics (SHs) [108] to model the 3D shape of the prostate.

In medical image processing, more sophisticated shape models can be integrated to provide more accurate segmentation [109-123]. ASMs, developed by Cootes et al. [95], are popular DMs that allow for a compact representation of an object's shape that adjusts for shape variance, but still maintain their general shape [124]. This method has been used extensively for TRUS prostate segmentation. Shen et al. [125] proposed an automated technique that utilized Gabor [126]based appearance features to guide an ASM for prostate segmentation. Another automated technique proposed by Betrouni et al. [127] was based on optimizing an ASM to segment the prostate. Zaim and Jankun [128] used an ASM, guided by extracted image appearance features to find the prostate boundary. Additionally, 
Yan et al. [129] proposed an ASM-based technique that incorporated a priori shape model [130] for segmentation. Also, Hodge et al. [131] proposed a semi-automated technique for $3 \mathrm{D}$ prostate segmentation by propagating $2 \mathrm{D}$ segmentations on a slice-by-slice basis.

Extensions of ASMs, such as active appearance models (AAM) [132], have also used for segmentation. For example, Ghose et al. [133] proposed an automated technique that utilized Haar-wavelet [134]-based features and a statistical shape model to guide an AAM for segmentation. Additionally, Ghose et al. [135] proposed an automated technique that utilized an AAM to segment the prostate based on appearance and shape information, derived using principle component analysis (PCA) [136]. Medina et al. [137] proposed another automated technique that used appearance and shape information to guide an AAM for 3D prostate segmentation.

In addition to edge detection and DM-based approaches, statistical-based methods [138-147] have been proposed for TRUS prostate segmentation, such as pixel classification and graph-cut [148] methods. In pixel classification techniques, each pixel is defined as object or non-object based on a set of extracted image features. An automated pixel classification technique was proposed by Mohamed et al. [149] that used Gabor [126]-based features and SVM [76] classification. Ghose et al. [150] performed 3D TRUS prostate segmentation using a graph-cut [148] method and an ASM. Table 2 summarizes the current in vivo studies for prostate segmentation from TRUS images with the validation data sets and achieved performance for each study. Overall, the segmentation of the prostate from TRUS images is a still a challenge due to its low contrast and low SNR. Therefore, there is a need for developing more accurate methods and more advanced capturing techniques to overcome these problems. Once the prostate region is determined, the next step is to extract diagnostics features from the prostate region in order to perform diagnosis. 
TABLE 2: Summary of in vivo studies for prostate segmentation from TRUS images, outlining the validation data sets, the segmentation method, and the experimental performance for each study.

\begin{tabular}{|c|c|c|c|}
\hline Study & In vivo Data & Method & Performance \\
\hline $\begin{array}{l}\text { Abolmaesumi } \\
\text { and Sirous- } \\
\text { pour [79] }\end{array}$ & 6 Images & $\begin{array}{l}\text { - Automated } \\
\text { - Edge Detec- } \\
\text { tion }\end{array}$ & - OAE: $2 \%$ \\
\hline $\begin{array}{l}\text { Sahba et al. } \\
{[81]}\end{array}$ & 19 Images & $\begin{array}{l}\text { - Automated } \\
\text { - Edge Detec- } \\
\text { tion }\end{array}$ & $\begin{array}{l}\text { - MAD: } 3.3 \pm 1.3 \\
\text { (pixels) } \\
\text { - Area Error: } 2.4 \pm \\
1.05 \%\end{array}$ \\
\hline $\begin{array}{l}\text { Zaim et } \\
\text { al. [128] }\end{array}$ & $\begin{array}{l}10 \text { Images (3 } \\
\text { Subjects) }\end{array}$ & $\begin{array}{l}\text { - Automated } \\
\text { - Active } \\
\text { Shape } \\
\text { Model }\end{array}$ & $\begin{array}{l}\text { - Mean Distance Er- } \\
\text { ror: } 15.3 \text { (pixels) } \\
\text { - OAE: } 5.0 \% \\
\text { - ACC: } 92 \%\end{array}$ \\
\hline $\begin{array}{l}\text { Shen. et al. } \\
{[125]}\end{array}$ & 8 Images & $\begin{array}{l}\text { - Automated } \\
\text { - Active } \\
\text { Shape } \\
\text { Model }\end{array}$ & $\begin{array}{l}\text { - } \text { MAD: } 3.20 \pm 0.87 \\
\text { (pixels) } \\
\text { - OAE: } 3.98 \pm 0.97 \\
\text { (\%) }\end{array}$ \\
\hline & & & Continued on next page \\
\hline
\end{tabular}


TABLE 2 - continued from previous page

\begin{tabular}{|c|c|c|c|}
\hline Study & In vivo Data & Method & Performance \\
\hline $\begin{array}{l}\text { Betrouni et al. } \\
\text { [127] }\end{array}$ & $\begin{array}{l}35 \text { Images (11 } \\
\text { Subjects) }\end{array}$ & $\begin{array}{l}\text { - Automated } \\
\text { - Active } \\
\text { Shape } \\
\text { Model }\end{array}$ & $\begin{array}{l}\text { - Mean Distance Er- } \\
\text { ror: } 2.55(\mathrm{~mm})\end{array}$ \\
\hline $\begin{array}{l}\text { Hodge et al. } \\
\text { [131] }\end{array}$ & 36 Volumes & $\begin{array}{l}\text { - Semi- } \\
\text { automated } \\
\text { - Active } \\
\text { Shape } \\
\text { Model } \\
\text { - 3D }\end{array}$ & $\begin{array}{l}\text { - } \text { MAD: } 1.09 \pm 0.49 \\
(\mathrm{~mm}) \\
\text { - } \text { AVE: } 3.28 \pm 3.16 \\
\\
(\%)\end{array}$ \\
\hline $\begin{array}{l}\text { Gong et } \\
\text { al. [103] }\end{array}$ & $\begin{array}{l}16 \text { Subjects } \\
\text { (125 Images) }\end{array}$ & $\begin{array}{l}\text { - Automated } \\
\text { - Curve fit- } \\
\text { ting }\end{array}$ & $\begin{array}{l}\text { - } \text { MAD: } 0.54 \pm 0.20 \\
(\mathrm{~mm}) \\
\text { - } \mathrm{HD}: 1.32 \pm 0.62 \\
(\mathrm{~mm})\end{array}$ \\
\hline $\begin{array}{l}\text { Badiei et al. } \\
\text { [105] }\end{array}$ & 17 Images & $\begin{array}{l}\text { - Automated } \\
\text { - Curve Fit- } \\
\text { ting }\end{array}$ & $\begin{array}{l}\text { - MAD: } 0.67 \pm 0.18 \\
(\mathrm{~mm}) \\
\text { - ACC: } 93 \% \\
\text { - SEN: } 97 \%\end{array}$ \\
\hline
\end{tabular}


TABLE 2 - continued from previous page

\begin{tabular}{|c|c|c|c|}
\hline Study & In vivo Data & Method & Performance \\
\hline $\begin{array}{l}\text { Mahdavi et } \\
\text { al. [106] }\end{array}$ & 40 Volumes & $\begin{array}{l}\text { - Semi- } \\
\text { automated } \\
\text { - Curve Fit- } \\
\text { ting } \\
\text { - 3D }\end{array}$ & - AVE: $5.82 \pm 4.15 \%$ \\
\hline $\begin{array}{l}\text { Yan et } \\
\text { al. [129] }\end{array}$ & 301 Images & $\begin{array}{l}\text { - Automated } \\
\text { - Active } \\
\text { Shape } \\
\text { Model }\end{array}$ & $\begin{aligned} \text { - } & \text { MAD: } 2.01 \pm 1.02 \\
& (\mathrm{~mm})\end{aligned}$ \\
\hline $\begin{array}{l}\text { Zhan and } \\
\text { Shen [102] }\end{array}$ & 3 Volumes & $\begin{array}{l}\text { - Automated } \\
\text { - Level-set } \\
\text { - 3D }\end{array}$ & $\begin{array}{l}\text { - MAD: } 0.81 \text { (voxels) } \\
\text { - Overlap Volume } \\
\text { Error: } 3.93 \% \\
\text { - Total Volume Er- } \\
\text { ror: } 1.5 \%\end{array}$ \\
\hline $\begin{array}{l}\text { Medina et al. } \\
\text { [137] }\end{array}$ & 95 Images & $\begin{array}{l}\text { - Automated } \\
\text { - Active } \\
\text { Shape } \\
\text { Model }\end{array}$ & $\begin{array}{l}\text { - MAD: } 3.58 \pm 1.49 \\
\text { (pixels) }\end{array}$ \\
\hline & & & Continued on next page \\
\hline
\end{tabular}


TABLE 2 - continued from previous page

\begin{tabular}{|c|c|c|c|}
\hline Study & In vivo Data & Method & Performance \\
\hline $\begin{array}{l}\text { Ghose et al. } \\
\text { [133] }\end{array}$ & 25 Images & $\begin{array}{l}\text { - Automated } \\
\text { - Active } \\
\text { Shape } \\
\text { Model }\end{array}$ & $\begin{array}{l}\text { - DSC: } 0.95 \pm 0.01 \\
\text { - } \mathrm{HD}: 5.08 \pm 1.18 \\
\text { (mm) } \\
\text { - MAD: } 1.48 \pm 0.36 \\
\text { (mm) }\end{array}$ \\
\hline $\begin{array}{l}\text { Ghose et al. } \\
\text { [135] }\end{array}$ & 23 Volumes & $\begin{array}{l}\text { - Automated } \\
\text { - Active } \\
\text { Shape } \\
\text { Model } \\
\text { - 3D }\end{array}$ & $\begin{array}{l}\text { - DSC: } 0.97 \pm 0.01 \\
\text { - MAD: } 0.49 \pm 0.20 \\
(\mathrm{~mm}) \\
\text { - HD: } 1.78 \pm 0.73 \\
(\mathrm{~mm}) \\
\text { - SPE: } 0.95 \pm 0.01 \\
\text { - SEN: } 0.99 \pm 0.00 \\
\text { - ACC: } 0.98 \pm 0.00\end{array}$ \\
\hline $\begin{array}{l}\text { Tutar et } \\
\text { al. [107] }\end{array}$ & 30 Volumes & $\begin{array}{l}\text { - Semi- } \\
\text { automated } \\
\text { - Spherical } \\
\text { Harmonics } \\
\text { - 3D }\end{array}$ & $\begin{array}{l}\text { - Average Volume } \\
\text { Overlap: } 83.5 \pm \\
4.2 \% \\
\text { - MAD: } 1.26 \pm 0.41 \\
\text { (mm) }\end{array}$ \\
\hline \multicolumn{4}{|c|}{$\begin{array}{l}\text { *ACC denotes accuracy. } \\
\text { *ROC denotes receiver operating characteristic. } \\
\text { *AUC denotes area under the ROC curve. }\end{array}$} \\
\hline
\end{tabular}


TABLE 2 - continued from previous page

\begin{tabular}{|c|c|c|c|}
\hline Study & In vivo Data & Method & Performance \\
\hline \multicolumn{4}{|c|}{ *AVE denotes average volume error. } \\
\hline \multicolumn{4}{|c|}{ *DSC denotes Dice's similarity coefficient. } \\
\hline \multicolumn{4}{|c|}{ *HD denotes Hausdorff distance error. } \\
\hline \multicolumn{4}{|c|}{${ }^{*} \mathrm{~F}-\mathrm{M}$ denotes F-Measure. } \\
\hline \multicolumn{4}{|c|}{ *MAD denotes mean absolute distance. } \\
\hline \multicolumn{4}{|c|}{ *PPV denotes positive predictive value. } \\
\hline \multicolumn{4}{|c|}{ *OAE denotes overlapping area error. } \\
\hline \multicolumn{4}{|c|}{ *RMSD denotes root mean squared distance. } \\
\hline \multicolumn{4}{|c|}{ *SEN denotes sensitivity. } \\
\hline \multicolumn{4}{|c|}{ *SPE denotes specificity. } \\
\hline
\end{tabular}

TRUS feature extraction and diagnosis: The most intuitive feature for classifying an image region is the intensity of the pixels/voxels inside the region. From the pixel/voxel intensities, several features can be obtained. For example, Gaussian statistics (mean and standard deviation) of pixel/voxels intensities are used as features in several TRUS studies [151-155]. The Nakagami distribution has also been used for extracting features from pixel intensities in various studies [153, 154]. More advanced features, such as the energy, entropy, correlation, and homogeneity, can be obtained using the gray level dependence matrix (GLDM) [156]. These features are frequently used in TRUS-based CAD systems, such as in [152, 157, 158]. Other intensity-based features can be obtained using the gray level difference vectors (GLDV) method [159]. This technique can be used to calculate the contrast, angular second moment, the entropy, the mean, and the inverse difference moment. These features were employed in the prostate CAD system proposed in [158].

In addition to these intensity-based features, several other TRUS features can be used in prostate cancer CAD systems. Examples of these include wavelet 
coefficients [160] and their polynomial fitting [160], which were used in [153-155], the autocorrelation coefficients [151], and a tumor's shape metric [151]. In addition, fractal texture features [161] and spectral features [162] were also used in prostate CAD systems, such as in [155] and [153-155], respectively. Another feature utilized is the total least square estimation of signal parameters [157], which is estimated via rotational invariance techniques (TLS-ESPRIT) [163, 164]. After extracting the diagnostics features from the TRUS images, the final step of a CAD system is to diagnose whether the prostatic a tissue is cancerous or non-cancerous using the selected features as an input. To perform this task, several classification methods can be used, such as SVM [76], linear discriminant analysis (LDA) [165, 166], K-means [167], K-nearest neighbors (kNN) [167], decision trees [167], Bayesian inference, and relevance vector machines (RVM) [168].

In the literature, several CAD systems have been developed to diagnose prostate cancer based on different extracted features from TRUS images and different classification techniques. For example, Maggio et al. [153,154] proposed an automated CAD system that utilized the tissue intensities, features extracted using the Nakagami distribution, Haralick textual features, and Unser textual features to classify prostate tissues as benign or malignant. Using an LDA classifier, this system had a sensitivity of $75 \pm 9 \%$, a specificity of $93 \pm 2 \%$, an accuracy of $93 \pm 2 \%$, and an AUC of $0.95 \pm 0.02$. Scebran et al. [155] proposed a three step automated CAD system. First, possible tumor ROIs were segmented using a combination of k-means and Bayesian pixel classification. Second, three types of feature were extracted from these ROIs: intensity, textural, and spectral parameters. The intensity parameters were extracted as the parameters of Gaussian and Nakagami distributions that model the visual appearance the image. The textural parameters were extracted using Unser, Gabor, and fractal [161] textural models. The spectral parameters were selected as the central frequency, mid-band and slope, and the polynomial fitting of the wavelet spectrum. Finally, classification of the ROIs 
was performed using SVM with a radial basis function kernel. The validation results showed that this method had an average specificity of $92 \%$ and an accuracy of $90 \%$. However, the sensitivity was considerably lower, having a value of $78 \%$. The authors report that this was caused by the system overestimating the size of possible tumors. Additionally, Mohamed et al. [149] proposed another automated CAD system that consists of three steps. First, ROIs were segmented by applying a Gabor filter [126] to the TRUS image and then performing multiresolution analysis $[169,170]$. Second, GLDM and GLDV features were extracted from these ROIs. Finally, these features were used to classify the ROIs. Two classifiers were found to be equally effective in this system, a decision tree and SVMs. Testing showed that using only GLDV features resulted in the highest classification accuracy. Validation with GLDV found both classifications had a sensitivity of $83.33 \%$, a specificity of $100 \%$, and an accuracy of $93.75 \%$. In [171], Mohamed et al. [171] extended the method in [149] by using features calculated using the TLS-ESPRIT spectral feature method [163,164]. The resulting feature vector was classified using SVM. The reported results showed the system had a sensitivity of $83.3 \%$, a specificity of $100 \%$, and an accuracy of $94.4 \%$. In the CAD system developed by Han et al. [151], the prostate was segmented and then the intensity information was used to find possible tumor ROIs. Four features were extracted for diagnosis: pixel intensity values, autocorrelation coefficients of image signals, tumor location, and the tumor shape. The shape of the tumor was quantified by how similar a possible tumor was to an ellipse. After these features were extracted, each ROI was classified using SVM. This technique showed a sensitivity and specificity of $92 \%$ and $95.6 \%$, respectively. A summary of the discussed CAD systems using in vivo TRUS images with their computational methods, validation data sets, and validation accuracy are given in Table 3. While these systems are effective, they are limited to extracting intensity and textural features from the images. For this reason, recent research has focused on MRI-based CAD systems to extract more sophisticated features in order to en- 
hance the accuracy of prostate cancer diagnosis.

TABLE 3: Summary of TRUS prostate cancer CAD systems on in-vivo data, including their prostate segmentation method, features, classifier, and experimental performance.

\begin{tabular}{|c|c|c|c|c|c|}
\hline Study & $\begin{array}{l}\text { In vivo } \\
\text { Data }\end{array}$ & $\begin{array}{l}\text { Prostate } \\
\text { Segmen- } \\
\text { tation }\end{array}$ & Features & Classifier & Performance \\
\hline $\begin{array}{l}\text { Han et } \\
\text { al. [151] }\end{array}$ & $\begin{array}{l}51 \text { Sub- } \\
\text { jects }\end{array}$ & $\begin{array}{l}\text { Otsu } \\
\text { Thresh- } \\
\text { old }\end{array}$ & $\begin{array}{l}\text { - Intensity } \\
\text { Gaussian } \\
\text { Model } \\
\text { - Auto- } \\
\text { correlation } \\
\text { Coeffi- } \\
\text { cients } \\
\text { - Tumor Lo- } \\
\text { cation } \\
\text { - Tumor } \\
\text { Shape }\end{array}$ & SVM & $\begin{array}{c}\text { - ACC: } \\
0.87 \\
\text { - SEN: } \\
0.92 \\
\text { - SPE: } \\
0.96\end{array}$ \\
\hline
\end{tabular}


TABLE 3 - continued from previous page

\begin{tabular}{|c|c|c|c|c|c|}
\hline Study & $\begin{array}{l}\text { In vivo } \\
\text { Data }\end{array}$ & $\begin{array}{l}\text { Prostate } \\
\text { Segmen- } \\
\text { tation }\end{array}$ & Features & Classifier & Performance \\
\hline $\begin{array}{l}\text { Llobert } \\
\text { et } \\
\text { al. [152] }\end{array}$ & $\begin{array}{l}303 \text { Sub- } \\
\text { jects }\end{array}$ & $\begin{array}{l}\text { Manual } \\
\text { ROIs }\end{array}$ & $\begin{array}{l}\text { - Intensity } \\
\text { Gaussian } \\
\text { Model } \\
\text { - GLDM }\end{array}$ & $\begin{array}{l}\text { kNN } \\
\text { and } \\
\text { HMM }\end{array}$ & $\begin{array}{l}\text { - } \mathrm{AUC} \\
(\mathrm{HMM}): \\
0.600 \\
\pm 0.7 \\
\text { - } \mathrm{AUC} \\
(\mathrm{kNN}): \\
0.601 \\
\pm 0.7\end{array}$ \\
\hline $\begin{array}{l}\text { Maggio } \\
\text { et } \\
\text { al. [154] }\end{array}$ & $\begin{array}{l}37 \text { Sub- } \\
\text { jects }\end{array}$ & None & $\begin{array}{l}\text { - Intensity } \\
\text { Gaussian } \\
\text { Model } \\
\text { - Intensity } \\
\text { Nakagai } \\
\text { Model } \\
\text { - Spectral } \\
\text { Features } \\
\text { - Wavelet } \\
\text { Coeffi- } \\
\text { cients }\end{array}$ & LDA & $\begin{array}{c}\text { - AUC: } \\
0.95 \\
\pm 0.02 \\
\text { - ACC: } \\
0.93 \\
\pm 0.02 \\
\text { - SEN: } \\
0.75 \\
\pm 0.09 \\
\text { SPE: } \\
0.93 \\
\pm 0.01\end{array}$ \\
\hline
\end{tabular}


TABLE 3 - continued from previous page

\begin{tabular}{|c|c|c|c|c|c|}
\hline Study & $\begin{array}{l}\text { In vivo } \\
\text { Data }\end{array}$ & $\begin{array}{l}\text { Prostate } \\
\text { Segmen- } \\
\text { tation }\end{array}$ & Features & Classifier & Performance \\
\hline $\begin{array}{l}\text { Scebran } \\
\text { et } \\
\text { al. [155] }\end{array}$ & $\begin{array}{l}37 \mathrm{Sub}- \\
\text { jects }\end{array}$ & $\begin{array}{l}\text { K-means } \\
\text { and } \\
\text { Bayes } \\
\text { Classifier }\end{array}$ & $\begin{array}{l}\text { - Spectral } \\
\text { Features } \\
\text { - Wavelet } \\
\text { Coeffi- } \\
\text { cients } \\
\text { - Fractal }\end{array}$ & SVM & $\begin{array}{l}\text { - SEN: } \\
0.78 \\
\text { - SPE: } \\
0.92 \\
\text { - ACC: } \\
0.90\end{array}$ \\
\hline $\begin{array}{l}\text { Mohamed } \\
\text { et } \\
\text { al. [157] }\end{array}$ & $\begin{array}{l}21 \quad \text { Sub- } \\
\text { jects }(33 \\
\text { Images })\end{array}$ & $\begin{array}{l}\text { Gabor } \\
\text { Filter }\end{array}$ & - GLDV & $\begin{array}{l}\text { SVM } \\
\text { and De- } \\
\text { cision } \\
\text { Tree }\end{array}$ & $\begin{array}{l}\text { - SEN: } \\
0.833 \\
\text { - SPE: } \\
1.000 \\
\text { - ACC: } \\
0.938\end{array}$ \\
\hline $\begin{array}{l}\text { Mohamed } \\
\text { et } \\
\text { al. [149] }\end{array}$ & 108 ROIs & $\begin{array}{l}\text { Gabor } \\
\text { Filter and } \\
\text { Multi- } \\
\text { resolution } \\
\text { Analysis }\end{array}$ & - ESPRIT & SVM & $\begin{array}{l}\text { - SEN: } \\
0.833 \\
\text { - SPE: } \\
1.000 \\
\text { - ACC: } \\
0.944\end{array}$ \\
\hline \multicolumn{6}{|c|}{$\begin{array}{l}\text { *ACC denotes accuracy. } \\
{ }^{*} \text { ROC denotes receiver operating characteristic. }\end{array}$} \\
\hline \multicolumn{6}{|c|}{ Continued on next page } \\
\hline
\end{tabular}


TABLE 3 - continued from previous page

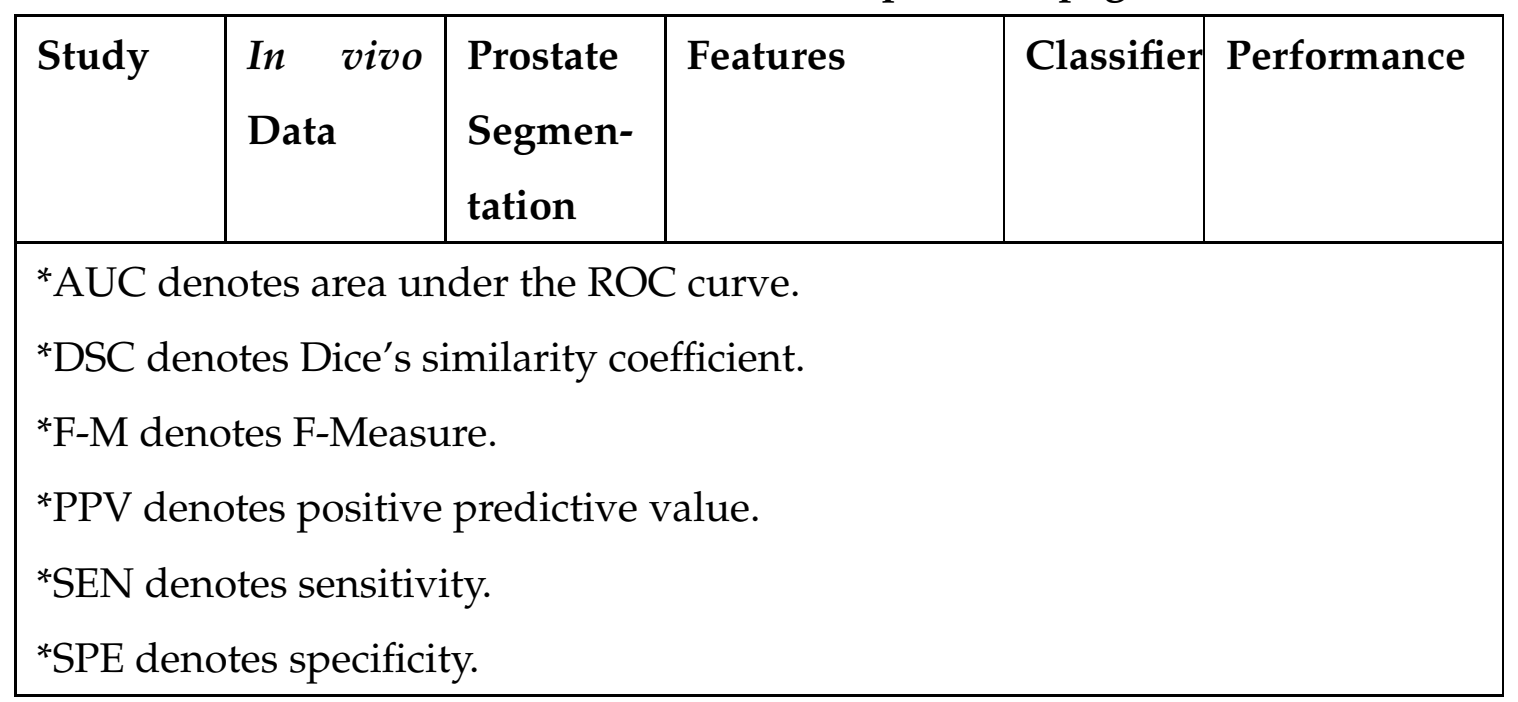

\section{MRI-based CAD systems}

Recent research studies focus on developing CAD systems for prostate diagnosis using MRI due to its ability to offer better soft tissue contrast. As with TRUS, these systems segment the prostate, extract features, and then perform diagnosis based on these features (see Fig 5). In this section, the segmentation techniques, the MRI extracted features, and the MRI-based CAD systems developed in the last 10 years are overviewed.

Prostate segmentation from in vivo MR images: MRI offers the best soft tissue contrast compared to the other image modalities used in prostate visualization. Therefore, the prostate can be defined more clearly in MR images than in TRUS images. However, segmentation is still challenging due to patient movement, intra-patient anatomical variations of the prostate shape and appearance, noise and inhomogeneities, and discontinuities of boundaries due to occlusions and similar visual appearance of adjacent structures. To address these challenges, many techniques have been developed to extract the prostate from MR images such as DM-based methods and statistical-based methods.

DMs have been applied extensively to segment the prostate from MRI data. 
For example, a hybrid 2D/3D ASM-based methodology for segmentation of the 3D MRI prostate data was proposed by Zhu et al. [172]. Toth et al. [173] presented an algorithm for the automatic segmentation of the prostate in multi-modal MRI (T2-MRI and magnetic resonance spectroscopy (MRS)). Their algorithm starts by isolating the region of interest (ROI) from MRS data. Then, an ASM within the ROI is used to obtain the final segmentation. Gao et al. [174] aligned the MR images before segmenting the prostate using a level-set guided by appearance information and a learned shape prior. Ghose et al. [175] used a similar approach that aligned T2-MRI data then an AAM was used to segment the prostate. Martin et al. [176] used a probabilistic anatomical atlas to constrain a DM-based framework for segmenting the prostate from 3D MR images. Allen et al. [177] proposed a framework for 3D prostate segmentation from T2-MRI based on voxel classification and a statistical shape model. Liu et al. [178] proposed a level-set technique guided by a shape prior and intensity information for 2D DWI prostate segmentation. Liu et al. [179] proposed a shape-based level-set method for 3D DWI prostate segmentation guided by an initial coarse segmentation.

Statistical-based techniques have also been used to segment the prostate from MRI data such as graph-cut [148] methods, random walk classification [180], and probabilistic anatomical atlases. For example, Ghose et al. [181] proposed a probabilistic graph-cut-based framework for 3D T2-MRI prostate segmentation based on a probabilistic atlas. Firjany et al. [147] proposed a Markov random field (MRF) image model [182-196] for 2D DCE-MRI prostate segmentation that combined a graph-cut approach with a prior shape model of the prostate and the visual appearance of the prostate image, modeled using a linear combination of discrete Gaussian (LCDG) [197-208] Their method was later extended in [209,210] to allow for 3D prostate segmentation from DCE-MRI volumes. The main limitation of graph-cut techniques is that they are prone to minimizing the size of the segmented region [211]. A Maximum A Posteriori (MAP) [212]-based framework was 
proposed by Makni et al. [213] to perform automated 3D MRI prostate segmentation using a MRF model [214] and statistical shape information. Similarly, Firjani et al. used a MAP-based method that incorporated an LCDG intensity model, an MRF spatial model, and a shape prior for 3D prostate segmentation from DWIMRI volumes [67,146,215]. Random walk classification [180] was used for MRI prostate segmentation by Khurd et al. [216]. Also, Klein et al. [217] presented an atlas-based segmentation approach to extract the prostate from MR images based on averaging the best atlases that match the image to be segmented. Another automated technique, proposed by Dowling et al. [218], used an automated atlas approach to segment the prostate region based on a Selective and Iterative Method for Performance Level Estimation (SIMPLE) [219]-based alignment technique.

In addition to DMs and statistical-based techniques, several other methods have been proposed to segment the prostate from MR images. Flores-Tapia et al. [220] proposed a semi-automated edge detection technique for MRI prostate segmentation based on a static wavelet transform [221] to locate the prostate edges. A semi-automated approach by Vikal et al. [222] used priori knowledge of the prostate shape to detect the contour in each slice and then refined them to form a 3D prostate surface. Table 4 summarizes the current in vivo studies for prostate segmentation from MRI images with the validation data sets and achieved performance for each study. In sum, a tremendous number of studies have been developed for the segmentation of prostate MRI data. However, prostate segmentation is still an ongoing area of research due to challenging prostate images that have different MRI noise sources, have poor image resolutions and diffused or occluded prostate boundaries. 
TABLE 4: Summary of in vivo studies for prostate segmentation from MRI, including their validation data sets, the segmentation method, and the experimental performance.

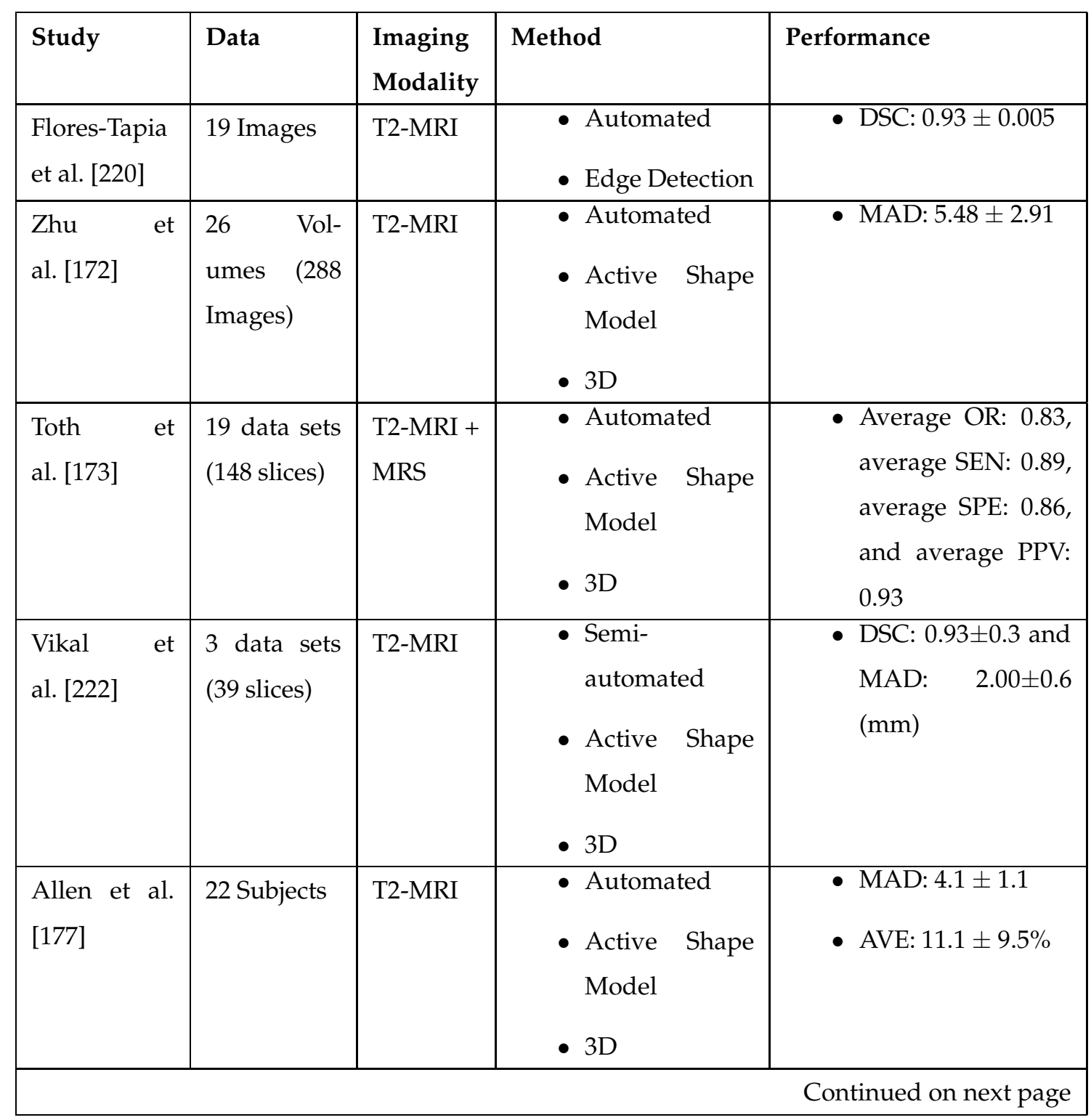


TABLE 4 - continued from previous page

\begin{tabular}{|c|c|c|c|c|}
\hline Study & Data & $\begin{array}{l}\text { Imaging } \\
\text { Modality }\end{array}$ & Method & Performance \\
\hline $\begin{array}{l}\text { Martin et al. } \\
\text { [176] }\end{array}$ & 36 Volumes & T2-MRI & $\begin{array}{l}\text { - Automated } \\
\text { - Active Shape } \\
\text { Model } \\
\text { - 3D }\end{array}$ & $\begin{array}{l}\text { - Median DSC: } 0.86 \\
\text { - Average Surface } \\
\text { Error: } 2.41(\mathrm{~mm})\end{array}$ \\
\hline $\begin{array}{l}\text { Ghose et al. } \\
\text { [175] }\end{array}$ & 15 Volumes & T2-MRI & $\begin{array}{l}\text { - Automated } \\
\text { - Active Shape } \\
\text { Model } \\
\text { - 3D }\end{array}$ & $\begin{array}{l}\text { - DSC: } 0.88 \pm 0.11 \\
\text { - HD: } 3.38 \pm 2.81 \\
\quad(\mathrm{~mm})\end{array}$ \\
\hline $\begin{array}{l}\text { Klein et al. } \\
\text { [217] }\end{array}$ & 38 Volumes & T2-MRI & $\begin{array}{l}\text { - Automated } \\
\text { - Atlas Registra- } \\
\text { tion } \\
\text { - 3D } \\
\end{array}$ & - Median DSC: 0.85 \\
\hline $\begin{array}{l}\text { Dowling et } \\
\text { al. [218] }\end{array}$ & 50 Volumes & T2-MRI & $\begin{array}{l}\text { - Automated } \\
\text { - Atlas Registra- } \\
\text { tion } \\
\text { - 3D } \\
\end{array}$ & $\begin{array}{l}\text { - Median DSC: } 0.86 \\
\text { - Average Surface } \\
\text { Error: } 2.00(\mathrm{~mm})\end{array}$ \\
\hline $\begin{array}{l}\text { Makni et al. } \\
\text { [213] }\end{array}$ & 12 Volumes & T2-MRI & $\begin{array}{l}\text { - Automated } \\
\text { - Graph Cut } \\
\text { - 3D }\end{array}$ & $\begin{array}{l}\text { - HD: } 9.94(\mathrm{~mm}) \\
\text { - AVE: } 0.163 \\
\text { - DSC: } 0.91\end{array}$ \\
\hline $\begin{array}{l}\text { Ghose et al. } \\
\text { [181] }\end{array}$ & 15 Volumes & T2-MRI & $\begin{array}{l}\text { - Automated } \\
\text { - Graph cut } \\
\text { - 3D }\end{array}$ & $\begin{array}{l}\text { - DSC: } 0.91 \pm 0.04 \\
\text { - HD: } 4.69 \pm 2.62 \\
(\mathrm{~mm})\end{array}$ \\
\hline $\begin{array}{ll}\text { Gao } & \text { et } \\
\text { al. [174] } & \end{array}$ & 33 Subjects & $\begin{array}{l}\text { T1-MRI } \\
\text { and } \\
\text { T2-MRI }\end{array}$ & $\begin{array}{l}\text { - Automated } \\
\text { - Atlas Registra- } \\
\text { tion } \\
\text { - Level-set }\end{array}$ & $\begin{array}{l}\text { - DSC: } 0.84 \pm 0.03 \\
\text { - HD: } 8.10 \pm 1.50 \\
(\mathrm{~mm})\end{array}$ \\
\hline
\end{tabular}


TABLE 4 - continued from previous page

\begin{tabular}{|c|c|c|c|c|}
\hline Study & Data & $\begin{array}{l}\text { Imaging } \\
\text { Modality }\end{array}$ & Method & Performance \\
\hline $\begin{array}{l}\text { Firjani et al. } \\
\text { [147] }\end{array}$ & $\begin{array}{l}14 \text { Volumes } \\
\text { (98 Images) }\end{array}$ & $\begin{array}{l}\text { DCE- } \\
\text { MRI }\end{array}$ & $\begin{array}{l}\text { - Automated } \\
\text { - Graph Cut }\end{array}$ & - AVE: $5.2 \pm 1.2 \%$ \\
\hline $\begin{array}{l}\text { Firjani et al. } \\
\text { [215] }\end{array}$ & $\begin{array}{ll}15 & \text { Sub- } \\
\text { jects } & (270 \\
\text { Volumes })\end{array}$ & $\begin{array}{l}\text { DCE- } \\
\text { MRI }\end{array}$ & $\begin{array}{l}\text { - } \text { Automated } \\
\text { - } 3 \mathrm{D} \\
\text { - MAP }\end{array}$ & $\begin{array}{l}\text { - DSC: } 0.92 \pm 0.004 \\
\text { - PPV: } 0.98 \pm 0.004 \\
\text { - SEN: } 0.85 \pm 0.004\end{array}$ \\
\hline $\begin{array}{ll}\text { Liu et } & \text { et } \\
\text { al. [178] }\end{array}$ & 10 Subjects & DWI & $\begin{array}{l}\text { - Automated } \\
\text { - Level-set }\end{array}$ & - DSC: $0.91 \pm 0.03$ \\
\hline $\begin{array}{ll}\text { Liu et } & \text { et } \\
\text { al. [179] }\end{array}$ & 10 Subjects & DWI & $\begin{array}{l}\text { - Automated } \\
\text { - Level-set } \\
\text { - } 3 \mathrm{D}\end{array}$ & $\begin{array}{l}\text { - } \text { DSC: } 0.810 \pm 0.05 \\
\text { - } \text { MAD: } 2.67 \pm 0.650 \\
(\mathrm{~mm}) \\
\text { - } \mathrm{HD}: 9.07 \pm 1.64 \\
(\mathrm{~mm})\end{array}$ \\
\hline $\begin{array}{l}\text { Firjani et al. } \\
\text { [67] }\end{array}$ & 28 Subjects & DWI & $\begin{array}{l}\text { - Automated } \\
\text { - } \mathrm{MAP} \\
\text { - } 3 \mathrm{D}\end{array}$ & $\begin{array}{l}\text { - } \text { DSC: } 0.991 \pm 0.004 \\
\text { - PPV: } 0.952 \pm 0.004 \\
\text { - SEN: } 0.816 \pm 0.004\end{array}$ \\
\hline $\begin{array}{l}\text { *ACC denotes } \\
\text { * ROC denotes } \\
\text { *AUC denotes } \\
\text { *AVE denotes } \\
\text { *DSC denotes } \\
\text { *HD denotes } \\
\text { *F-M denotes } \\
\text { * MAD denote } \\
{ }^{*} \text { PPV denotes } \\
\text { *OAE denotes } \\
\text { * RMSD denot } \\
{ }^{*} \text { SEN denotes } \\
\text { *SPE denotes }\end{array}$ & $\begin{array}{l}\text { accuracy. } \\
\text { receiver opera } \\
\text { area under th } \\
\text { average volum } \\
\text { Dice's similari } \\
\text { Hausdorff dista } \\
\text { F-Measure. } \\
\text { mean absolut } \\
\text { positive predic } \\
\text { overlapping a } \\
\text { s root mean sc } \\
\text { sensitivity. } \\
\text { pecificity. }\end{array}$ & $\begin{array}{l}\text { ing charact } \\
\text { ROC curve } \\
\text { error. } \\
\text { coefficient } \\
\text { ce error. } \\
\text { distance. } \\
\text { ive value. } \\
\text { ea error. } \\
\text { tared distar }\end{array}$ & istic. & \\
\hline
\end{tabular}

MRI feature extraction and diagnosis: Just as in TRUS-based CAD systems, 
MRI-based systems extract features in order to detect cancerous tumors. These features can be extracted from any MR image modality used in prostate CAD systems, e.g., T1-MRI, T2-MRI, DCE, and DWI MRI. Several proposed CAD systems in the literature have used multi-parametric MRI, a combination of multiple MRI modalities, to increase the number and quality of the features that the systems can utilize. Below, the common features extracted from each of these MRI modalities as well as the basic CAD systems developed in the last 10 years using these modalities are overviewed.

T2-MRI-based diagnostic systems extract several features from T2-MRI for classifying a prostate region as cancerous or noncancerous. These features include the pixel/voxel intensity values of T2-MR images [69-71,73,74, 223-228]. In addition, the 25 percentile [70], the variance and entropy of the T2-MRI intensities [71], the 2D [71] and 3D [223] intensity gradients, and the T2-MRI image texture [223] are commonly exploited as candidate features to discriminate between malignant and nonmalignant prostate tissues. In addition to pixel/voxel intensities, image filters were frequently used in order to extract features from T2-MRI. Image filtering applies a transform that maps each pixel/voxel on the image to a new value, from which new features can be extracted, such as the mean, standard deviation, average deviation, and median of the intensities of a pixel's neighbors [71,229]. Several image filters, such as the Gabor filter [126] and the Sobel filter [230], were used for feature extraction in [223, 225,227] and [71], respectively. Another T2-MRI feature is the relaxation time, the time it takes for protons to revert to their original energy state after the magnetic pulse created by an MRI machine. This feature was used in [223, 225].

Several T2-MRI CAD systems have been developed based on the extracted features from the baseline MRI methodology for prostate cancer detection (the initial image type used in prostate cancer CAD systems, i.e., T2-MRI). The main features extracted from T2-MRI images are signal intensities and texture-based fea- 
tures. These values have been utilized in multiple T2-MRI systems. One such semi-automated CAD system was proposed by Madabhushi et al. [223]. Pixels inside manually selected regions were labeled as tumourous or non-tumourous using a Bayesian classifier. The classification was then performed using a large set of features that included: gray levels statistics (intensity values, mean, and standard deviation), intensity gradient, and Gabor filter features. This pixel classification technique had a sensitivity of $42.35 \%$, a specificity of $97.25 \%$ and a PPV of $42.85 \%$. This system was improved in [227]. The Bayesian classifier was replaced with a kNN classifier that was built using Bayesian learners. This modified system had an AUC of 0.957. An automated T2-MRI CAD system was developed by Lopes et al. [228]. Each pixel in the image was labeled as either cancerous or non-cancerous based on their features. The fractal dimension and the multifractal spectrum calculated using a multifractional Browninan motion model were used as sources of features. Two classifiers were trained, SVM and AdaBoost [231]. The sensitivity and the specificity were $83 \%$ and $91 \%$ for SVM and $85 \%$ and $93 \%$ for AdaBoost.

DCE-MRI-based diagnostic systems were developed for prostate cancer diagnosis for several reasons. The addition of a contrast agent helps to distinguish objects of interest in MR images. In addition, the diffusion of the contrast agent can be used to add two common sources of DCE-MRI features, parametric (pharmacokinetic) and nonparametric parameters, in addition the intensity information. Pharmacokinetic parameters are measures of the kinetics of contrast agents through an organ in a DCE-MR image. The three standard pharmacokinetic parameters are the volume transfer constant $\left(K^{\text {trans }}\right)$, the extravascular extracellular space fractional volume $\left(v_{e}\right)$, and the rate constant $\left(k_{e p}\right)$ [232]. These parameters have been used as features in several DCE-MRI prostate cancer CAD systems [225, 229]. In addition, these features have also been used in many multi-parametric MRI CAD systems $[69-71,73,74,225,226]$. Specifically, the 75 percentile $K^{\text {trans }}$ value $[70-73,225]$, the mean $k_{e p}[69,225]$, the 75 percentile $k_{e p}[72,233]$, and the 
75 percentile $v_{e}$ [70] have been utilized as discriminating features.

In addition to the pharmacokinetic parameters, established dynamic perfusion analysis of extracellular extravascular agents, such as gadolinium agents, have also used empirical measures, including peak signal intensity, time-to-peak, wash-in slope, wash-out slope, and Area Under the Gadolinium Curve (AUGC). The time-to-peak is defined as the time from the injection of the contrast agent until the peak intensity is observed. The wash-in rate is defined as the maximum change in intensity during the time between the start of the inflow of the contrast agent and the time where the highest signal intensity is recorded [234]. This feature was used in $[73,229,235,236]$. The wash-out rate is defined as the maximum change in intensity during the time the highest signal intensity is recorded and a specified end time [237] and was used in the system proposed in $[235,236]$. The gadolinium curve is the plot of the gadolinium concentration versus time and the AUGC is the area under this curve [238]. This feature was used in the CAD system proposed in [71].

Based on the extracted features from DCE-MRI, several DCE-MRI CAD systems were developed for prostate cancer diagnosis. For example, Viswanath et al. [224] proposed a semi-automated DCE-MRI-based system, where the prostate was segmented using an ASM initialized by a manually placed bounding-box and then guided by image intensity, image texture and mutual information. To extract diagnostics features, local linear embedding (LLE) [239] was used to create a feature vector using local neighborhood intensities. K-means clustering was then used to classify the pixels within the segmented prostate as tumorous or non-tumourous. Validation showed that this system had a sensitivity of $41.73 \%$, a specificity of $84.54 \%$, and an accuracy of $77.20 \%$. A study by Engelbrecht et al. [240] used DCEMRI to evaluate which MRI parameters would result in optimal discrimination of prostatic carcinoma from normal $\mathrm{PZ}$ and $\mathrm{CZ}$ of the prostate. Using the ROC curves, their study concluded that the relative peak enhancement was the most accurate 
perfusion parameter for cancer detection in the $\mathrm{PZ}$ and $\mathrm{CZ}$ of the gland. Additionally, a semi-automated CAD system by Kim et al. [241] demonstrated that parametric imaging of the wash-in rate was more accurate for the detection of prostate cancer in the PZ than was $T_{2}$-MRI alone. However, they also observed significant overlap between the wash-in rate for cancer and normal tissue in the TZ. Fütterer et al. [242] developed a CAD system to compare the accuracies of $T_{2}$-MRI, DCEMRI, and MRS imaging for prostate cancer localization. The results showed higher accuracy in DCE-MRI than were achieved with $T_{2}$-MRI in prostate cancer localization. A similar study was conducted by Rouvière et al. [243] for the detection of postradiotherapy recurrence of prostate cancer. Their study also concluded that DCE-MRI possesses the ability to depict the intraprostatic distribution of recurrent cancer after therapy more accurately and with less inter-observer variability than $T_{2}$-MRI. Ocak et al. [244] developed a CAD system using PK analysis for prostate cancer diagnostics in patients with biopsy-proven lesions. In their framework, the $K^{\text {trans }}$, the $k_{\mathrm{ep}}$, the $v_{\mathrm{e}}$, and the area under the gadolinium concentration curve were determined and compared for cancer, inflammation, and healthy peripheral. Their results showed improvement in prostate cancer specificity using the $K^{\text {trans }}$ and $k_{\text {ep }}$ parameters over that obtained using conventional $T_{2}$-MRI. Puech et al. $[235,236]$ developed a semi-automated dynamic MRI-based CAD system for the detection of prostate cancer. Candidate lesion ROIs were selected either manually or by using a region growing technique initiated by a user-selected seed point. Lesions are classified as benign, malignant or indeterminate based on the analysis of the median wash-in and wash-out values. Their CAD system demonstrated a sensitivity and specificity of $100 \%$ and $45 \%$ for the PZ, and sensitivity and specificity of $100 \%$ and $40 \%$ for the TZ. Sung et al. [229] proposed another semi-automated system where ROIs were manually selected. These were then classified as cancerous or non-cancerous using $K_{\text {trans }}, k_{e p}, v_{e}$, wash-in rate, wash-out rate, and time-to-peak values. Testing showed that the system had a sensitivity of $90 \%$, specificity of $77 \%$, 
and accuracy of $83 \%$. In [245] and [233], Vos et al. proposed a semi-automated system and an automated system, respectively. In both, possible cancerous tumors in the PZ were classified as either tumorous or non-tumorous. ROIs were manually selected in the first system [245] and selected using a combination of an Otsu threshold segmentation [246] and a Hessian-based blob detection method [247] in the second system [233]. In both systems, the ROIs were then classified using the pharmacokinetic parameters and an SVM classifier. The average accuracies of $88 \%$ and $80 \%$ were shown for the semi-automated and automated approaches, respectively. However, these techniques were only capable of detecting and classifying tumors in the PZ and not the rest of the prostate.

Another automated DCE-MRI CAD system was proposed by Firjani et al. [248]. The first step in this system was performing probabilistic segmentation using the MAP algorithm and image intensity, spatial information modeled using an MRF, and a shape prior. The wash-in and wash-out curves were then used as sources of features for classification with a $\mathrm{kNN}$ classifier. This technique had an accuracy of $100 \%$ using a data set of 21 subjects.

DWI-MRI-based diagnostic systems acquire images at varying b-values (i.e. magnetic field strengths). This allows the Apparent Diffusion Coefficient (ADC) and other diagnostics features to be extracted. The ADC, a common intensitybased feature for DWI, is a measure of the impedance of water diffusion and is determined by evaluating the difference between two diffusion weighted images taken at different magnetic field strengths (e.g. b-values). The ADC values at each pixel/voxel are known as the ADC maps. They have been shown to be effective in differentiating between prostates containing cancerous tumors and those that do not [249]. In addition, it was shown that cancerous regions have a lower average ADC than non-cancerous regions [249]. Consequently, ADC maps have been used as a source of features in several MRI prostate cancer CAD systems [67, 69$73,225,226]$. The mean ADC $[69,70]$ and the median ADC value [71] are also com- 
mon features for prostate cancer diagnosis. In addition, the 25 percentile ADC value [70-72], and the 10 percentile ADC value [73] are popular features. Also, a Sobel filter was applied to the ADC map to extract additional features in [71]. T2 shine-through and T2 wash-out represent two additional DWI features. These features measure how much the intensity of a pixel/voxel changes between two DWI images acquired at different b-values. Typically, a b-value of 0 (i.e. T2-MRI) is used as a baseline and compared to a second, higher b-value. The intensities of these images are often referred to as $S_{0}$ and $S_{1}$, respectively. Shine-through occurs when the intensity increases drastically with an increase in b-value, whereas wash-out occurs when the intensity decreases drastically with an increase in b-value [250]. The change in the intensity has been used as a feature for CAD systems that utilize DWI $[70,72]$. Once a combination of these features are selected to form the feature space of a CAD system, classification can be performed. For example, Firjani et al. $[67,145]$ developed a CAD system for prostate diagnosis using DWI-MRI. The prostate is automatically segmented based on a prior shape, spatial interactions, and appearance information. Possible tumor locations were then found using a level set DM. The average DWI intensity at b-values of 800 and $0 \mathrm{~s} / \mathrm{mm} 2$ and the mean value of the ADC map were then extracted from these locations. Finally, a kNN classifier labeled benign and malignant regions of the prostate. Validation testing showed that the system had an accuracy of $100 \%$ using a dataset of 28 subjects, 13 of which were used for training and 15 for testing.

Multi-parametric-based diagnostic systems for prostate cancer use several MRI imaging modalities in conjunction as input data. This allows systems to select the most meaningful features from any of the modalities. These systems have used different combinations of MRI modalities and features. For example, T2-MRI and DCE-MRI were used as inputs in a semi-automated system proposed by Vos et al. [225]. This system classified manually-delineated ROIs in the PZ as malignant or benign using T2-MRI intensities, T2-MRI relaxation time, and pharmacoki- 
netic parameters as features. This approach showed an accuracy of $89 \%$ using an SVM classifier. Ampeliotis et al. [251] proposed a another semi-automated multiparametric CAD system that used $T_{2}$-MRI and DCE-MRI. The $T_{2}$-MRI pixel intensities and the four low-frequency coefficients of the discrete cosine transform were used as features and probabilistic neural networks were employed as the classifier. Based on the ROC analysis (AUC of 0.898), their study concluded that the fused $T_{2}$-MRI and dynamic MRI features outperform the use of either modality's features alone. Another semi-automated system that utilized T2-MRI and DCE-MRI as input was developed by Viswanath et al. [68]. An ASM model was initialized by a manually placed bounding-box and then guided by image intensity, image texture and mutual information to segment the prostate region. After segmentation, prostate tissues were classified as cancerous or non-cancerous using a random forest, which is made of multiple decision trees that vote on the classification. Classification integrated three features: T2 intensity, textual, and pharmacokinetic parameters. The system validation showed that the integration of both modalities (AUC of 0.815 ) has a better performance of either individual modalities ( 0.704 for $T_{2}$-MRI and 0.682 for DCE-MRI).

Haider et al. [252] developed a semi-automated system that utilized T2-MRI and DWI MRI. T2-MRI intensities and ADC values were extracted from manually delineated ROIs. These regions were then classified using the maximum likelihood method assuming a bivariate Gaussian distribution for benign and malignant classes. The system showed a sensitivity of $81 \%$, a specificity of $84 \%$, a PPV of $75 \%$, and an accuracy of $83 \%$. Chan et al. [166] developed a semi-automated approach using T2-MRI, $T_{2}$-mapping, and line scan DWI to detect possible PZ prostate tumors. Both statistical maps and textural features were obtained from manually selected ROIs. Then, a SVM and a linear discriminant analysis (LDA) classifiers were employed for the classification. Their systems resulted in an AUC of $0.839 \pm 0.064$ and $0.761 \pm 0.043$, respectively. 
The combination of T2-MRI, DCE-MRI, and DWI MRI is a common multiparametric input. In [74], Shah et al. [74] developed an automated CAD system utilizing these modalities. In this system, prostate segmentation was performed using a k-means clustering approach based on the pixel's T2, $K^{\text {trans }}, k_{e p}$, and ADC values. Then, an SVM technique was implemented to create a cancer probability map for each prostate pixel using those features in order to perform the final classification. The system achieved a sensitivity of $90 \%$, a specificity of $90 \%$, and a precision of 90\%. Another semi-automated multi-parametric system by Peng et al. [73] utilized $T_{2}$-MRI, DCE-MRI, and DWI-MRI. Candidate features, including the $T_{2}$-MRI intensity skew, the $K^{\text {trans }}$, and the average and $10^{\text {th }}$ percentile ADC, were calculated from a manually-selected ROI. Then, an LDA classifier was used to differentiate prostate cancer from normal tissue in those ROIs. Their CAD system concluded that the best diagnostic performance (AUC of $0.95 \pm 0.02$, sensitivity of $82.0 \%$, and specificity of $95.3 \%$ ) is obtained by combining the $10^{\text {th }}$ percentile ADC, average $\mathrm{ADC}$, and T2-MRI intensity skewness features. Another CAD system was proposed by Litjens et al. [69] using T2-MRI, DCE-MRI, and DWI MRI. The prostate is segmented using an ASM. In order to classify the segmented prostate voxels, the ADC, $K^{\text {trans }}$, and $k_{\text {ep }}$ parameters were estimated and a SVM classifier with a radial basis function kernel was used. The validation results showed a sensitivity of $74.7 \%$ and $83.4 \%$ with seven and nine false positives per patient, respectively. Vos et al. [70] utilized an automated CAD system for the detection of prostate cancer. Just as in [69], the prostate was segmented using an ASM-based technique. Then, multiple ROIs were located within the segmented prostate using peak and mean neighborhood intensity and ADC values. These values and the differences between the peak and the mean were again used as features for ROI classification. In addition, the 25 percentile T2, 25 percentile ADC, 25 percentile wash-out, 50 percentile $T_{1}, 75$ percentile $K^{\text {trans }}$, and 75 percentile $v_{\mathrm{e}}$ were also used as features. The resulting feature vector was classified using an LDA classifier. This system 
had an AUC of $0.83 \pm 0.20$. A maximum AUC of 0.88 was reported for high-grade tumors, but the system had difficulty classifying lower grade tumors, achieving a maximum AUC of 0.74 .

In addition, several automated CAD systems that directly segment tumors have also been proposed. Liu et al. [253] proposed an automated approach that utilized fuzzy MRF modeling for prostate segmentation from multi-parametric MRI (T2-MRI, DCE-MRI, and DWI MR images). Their framework exploited T2MR image intensities, pharmacokinetic (PK) parameter $k_{\mathrm{ep}}$, and apparent diffusion coefficient (ADC) values in a Bayesian approach to label prostate pixels as cancerous or non-cancerous. The labeled pixels were then clustered using the kmeans algorithm. The system had a specificity of $89.58 \%$, sensitivity of $87.50 \%$, accuracy of $89.38 \%$, and a DSC of $62.2 \%$. A similar approach developed by Artan et al. [254] located cancerous regions using cost-sensitive support vector machine (SVM). Prostate segmentation was performed using a conditional random field and the same three features as in [253] were utilized for classification. The DSC for prostate localization and segmentation was $0.46 \pm 0.26$, and the area under the receiver operator characteristic (ROC) curves $\left(A_{z}\right)$ of the classification was $0.79 \pm 0.12$. Ozer et al. [226] also developed a technique that directly segmented prostate cancers using the same three features in $[253,254]$. Both the SVM and RVM [168] classifiers were used and the system showed a specificity of 0.78 and a sensitivity of 0.74 for RVM and 0.74 and 0.79 for SVM. A summary of the discussed systems along with their computational methods, validation data sets, and validation accuracies are given in Table 5. As shown, the use of multi-parametric MRI in CAD systems increases the possible number of features used for prostate cancer diagnosis. Consequently, using multiple MRI modalities has become the focus area for many research studies for prostate cancer diagnosis. 
TABLE 5: Summary of prostate cancer CAD systems using invivo MRI, including their imaging modality, prostate segmentation method, features, classifier, and experimental performance.

\begin{tabular}{|c|c|c|c|c|c|c|}
\hline Study & Data & $\begin{array}{l}\text { Imaging } \\
\text { Modal- } \\
\text { ity }\end{array}$ & $\begin{array}{l}\text { Prostate } \\
\text { Seg- } \\
\text { men- } \\
\text { tation }\end{array}$ & Features & Classifier & Performance \\
\hline $\begin{array}{l}\text { Mada- } \\
\text { bhushi } \\
\text { et } \\
\text { al. [223] }\end{array}$ & $\begin{array}{l}5 \text { Sub- } \\
\text { jects }\end{array}$ & $\begin{array}{l}\text { T2- } \\
\text { MRI }\end{array}$ & $\begin{array}{l}\text { Manual } \\
\text { ROIs }\end{array}$ & $\begin{array}{l}\text { - } \mathrm{T} 2 \text { In- } \\
\text { tensity } \\
\text { - } 3 \mathrm{D} \text { T2 } \\
\text { Inten- } \\
\text { sity } \\
\text { Gradi- } \\
\text { ent } \\
\text { - Gabor } \\
\text { Filter }\end{array}$ & $\begin{array}{l}\text { Bayes } \\
\text { classifier }\end{array}$ & $\begin{array}{l}\text { - SEN: } \\
0.4285 \\
\text { - SPE: } \\
0.9725 \\
\text { - PPV: } \\
0.4285\end{array}$ \\
\hline $\begin{array}{l}\text { Mada- } \\
\text { bhushi } \\
\text { et } \\
\text { al. [227] }\end{array}$ & $\begin{array}{l}5 \text { Sub- } \\
\text { jects (33 } \\
\text { Images) }\end{array}$ & $\begin{array}{l}\text { T2- } \\
\text { MRI }\end{array}$ & $\begin{array}{l}\text { Manual } \\
\text { ROIs }\end{array}$ & $\begin{array}{l}\text { - } \text { T2 In- } \\
\text { tensity } \\
\text { - } 3 \mathrm{D} \mathrm{T2} \\
\text { Inten- } \\
\text { sity } \\
\text { Gradi- } \\
\text { ent } \\
\text { - Gabor } \\
\text { Filter }\end{array}$ & $\begin{array}{l}\mathrm{kNN} \text { and } \\
\text { Bayesian }\end{array}$ & $\begin{array}{r}\text { - } \mathrm{AUC} \text { : } \\
0.957\end{array}$ \\
\hline
\end{tabular}


TABLE 5 - continued from previous page

\begin{tabular}{|c|c|c|c|c|c|c|}
\hline Study & Data & $\begin{array}{l}\text { Imaging } \\
\text { Modal- } \\
\text { ity }\end{array}$ & $\begin{array}{l}\text { Prostate } \\
\text { Seg- } \\
\text { men- } \\
\text { tation }\end{array}$ & Features & Classifier & Performance \\
\hline $\begin{array}{l}\text { Lopes et } \\
\text { al. [228] }\end{array}$ & $\begin{array}{l}17 \text { Sub- } \\
\text { jects }\end{array}$ & $\begin{array}{l}\text { T2- } \\
\text { MRI }\end{array}$ & None & $\begin{array}{l}\text { Fractal } \\
\text { Dimen- } \\
\text { sion } \\
\text { - Multi- } \\
\text { fractional } \\
\text { Brow- } \\
\text { nian } \\
\text { Motion }\end{array}$ & AdaBoost & $\begin{array}{c}\text { - SEN: } \\
0.85 \\
\text { - SPE: } \\
0.93\end{array}$ \\
\hline $\begin{array}{l}\text { Engel- } \\
\text { brecht et } \\
\text { al. [240] }\end{array}$ & $\begin{array}{l}36 \text { sub- } \\
\text { jects }\end{array}$ & $\begin{array}{l}\text { DCE- } \\
\text { MRI }\end{array}$ & $\begin{array}{l}\text { Manual } \\
\text { ROIs }\end{array}$ & $\begin{array}{l}\text { - } \text { Onset } \\
\text { time } \\
\text { - Time to } \\
\text { peak } \\
\text { - Peak } \\
\text { en- } \\
\text { hance- } \\
\text { ment } \\
\text { - T2 } \\
\text { washout } \\
\text { - T2 re- } \\
\text { laxation } \\
\text { rate }\end{array}$ & $\mathrm{N} / \mathrm{A}$ & $\begin{array}{l}\text { AUC } \\
\text { PZ: } \\
0.93 \\
\text { - AUC } \\
\text { CZ: } \\
0.83\end{array}$ \\
\hline
\end{tabular}


TABLE 5 - continued from previous page

\begin{tabular}{|c|c|c|c|c|c|c|}
\hline Study & Data & $\begin{array}{l}\text { Imaging } \\
\text { Modal- } \\
\text { ity }\end{array}$ & $\begin{array}{l}\text { Prostate } \\
\text { Seg- } \\
\text { men- } \\
\text { tation }\end{array}$ & Features & Classifier & Performance \\
\hline $\begin{array}{l}\text { Rouvière } \\
\text { et } \\
\text { al. [243] }\end{array}$ & $\begin{array}{l}22 \text { sub- } \\
\text { jects }\end{array}$ & $\begin{array}{l}\text { T2- } \\
\text { MRI } \\
\text { vs. } \\
\text { DCE- } \\
\text { MRI }\end{array}$ & $\begin{array}{l}\text { Manual } \\
\text { ROIs }\end{array}$ & - $\mathrm{N} / \mathrm{A}$ & $\begin{array}{l}\text { Evaluation } \\
\text { and scor- } \\
\text { ing by } \\
\text { three } \\
\text { inde- } \\
\text { pendent } \\
\text { readers. } \\
\text { The MRI } \\
\text { scoring } \\
\text { results } \\
\text { were cor- } \\
\text { related } \\
\text { against } \\
\text { biopsy } \\
\text { results } \\
\text { in } 10 \\
\text { prostate } \\
\text { sectors. }\end{array}$ & $\begin{array}{l}\text { - ACC: } \\
0.59 \\
(\mathrm{~T} 2- \\
\text { MRI), } \\
\text { - ACC: } \\
0.75 \\
\text { (DCE- } \\
\text { MRI). }\end{array}$ \\
\hline
\end{tabular}


TABLE 5 - continued from previous page

\begin{tabular}{|c|c|c|c|c|c|c|}
\hline Study & Data & $\begin{array}{l}\text { Imaging } \\
\text { Modal- } \\
\text { ity }\end{array}$ & $\begin{array}{l}\text { Prostate } \\
\text { Seg- } \\
\text { men- } \\
\text { tation }\end{array}$ & Features & Classifier & Performance \\
\hline $\begin{array}{l}\text { Kim et } \\
\text { al. [241] }\end{array}$ & $\begin{array}{l}53 \text { sub- } \\
\text { jects }\end{array}$ & $\begin{array}{l}\text { T2- } \\
\text { MRI } \\
\text { vs. } \\
\text { DCE- } \\
\text { MRI }\end{array}$ & $\begin{array}{l}\text { Manual } \\
\text { ROIs }\end{array}$ & $\begin{array}{l}\text { - Wash- } \\
\text { in rate }\end{array}$ & $\begin{array}{l}\text { A cut-off } \\
\text { threshold } \\
\text { selected } \\
\text { by a ra- } \\
\text { diologist } \\
\text { differ- } \\
\text { entiate } \\
\text { cancer } \\
\text { from } \\
\text { normal } \\
\text { tissue }\end{array}$ & $\begin{array}{l}\text { - ACC: } \\
0.62 \\
(\mathrm{~T} 2- \\
\text { MRI), } \\
\text { - ACC: } \\
0.88 \\
\text { (DCE- } \\
\text { MRI) }\end{array}$ \\
\hline $\begin{array}{l}\text { Fütterer } \\
\text { et } \\
\text { al. [242] }\end{array}$ & $\begin{array}{l}34 \text { sub- } \\
\text { jects }\end{array}$ & $\begin{array}{l}\text { T2- } \\
\text { MRI, } \\
\text { vs. } \\
\text { MRS, } \\
\text { vs. } \\
\text { DCE- } \\
\text { MRI }\end{array}$ & $\begin{array}{l}\text { Manual } \\
\text { ROIs }\end{array}$ & $\begin{array}{l}\text { - } v_{\mathrm{e}} \\
\text { - } k_{\mathrm{ep}} \\
\text { - } K^{\text {trans }} \\
\text { - wash- } \\
\text { out } \\
\text { slopes. }\end{array}$ & $\begin{array}{l}\text { Evaluation } \\
\text { and scor- } \\
\text { ing of the } \\
\text { selected } \\
\text { features } \\
\text { by two } \\
\text { inde- } \\
\text { pendent } \\
\text { radiolo- } \\
\text { gists }\end{array}$ & $\begin{array}{l}\text { - AUC: } \\
0.68 \\
\text { (T2- } \\
\text { MRI) } \\
\text { - AUC: } \\
0.91 \\
\text { (DCE- } \\
\text { MRI) } \\
\text { - AUC: } \\
0.80 \\
\text { (MRS) }\end{array}$ \\
\hline
\end{tabular}


TABLE 5 - continued from previous page

\begin{tabular}{|c|c|c|c|c|c|c|}
\hline Study & Data & $\begin{array}{l}\text { Imaging } \\
\text { Modal- } \\
\text { ity }\end{array}$ & $\begin{array}{l}\text { Prostate } \\
\text { Seg- } \\
\text { men- } \\
\text { tation }\end{array}$ & Features & Classifier & Performance \\
\hline $\begin{array}{l}\text { Ocak et } \\
\text { al. [244] }\end{array}$ & $\begin{array}{l}50 \text { sub- } \\
\text { jects }\end{array}$ & $\begin{array}{l}\text { T2- } \\
\text { MRI } \\
\text { vs. } \\
\text { DCE- } \\
\text { MRI }\end{array}$ & $\begin{array}{l}\text { Manual } \\
\text { ROIs }\end{array}$ & $\begin{array}{l}\text { - } v_{\mathrm{e}} \\
\text { - } K^{\text {trans }} \\
\text { - } k_{\mathrm{ep}} \\
\text { - } v_{\mathrm{e}} \\
\text { - The } \\
\text { area un- } \\
\text { der the } \\
\text { gadolin- } \\
\text { ium } \\
\text { concen- } \\
\text { tration } \\
\text { curve }\end{array}$ & $\begin{array}{l}\text { Logistic } \\
\text { regres- } \\
\text { sion } \\
\text { modeling }\end{array}$ & $\begin{array}{l}\text { • For T2- } \\
\text { MRI } \\
\text { SEN: } \\
0.94, \\
\text { SPE: } \\
0.37, \\
\text { PPV: } \\
50, \text { and } \\
\text { NPV: } \\
0.89, \\
\text { • For } \\
\text { DCE- } \\
\text { MRI } \\
\text { SEN: } \\
0.73, \\
\text { SPE: } \\
0.88, \\
\text { PPV: } \\
75, \text { and } \\
\text { NPV: } \\
0.75\end{array}$ \\
\hline
\end{tabular}


TABLE 5 - continued from previous page

\begin{tabular}{|c|c|c|c|c|c|c|}
\hline Study & Data & $\begin{array}{l}\text { Imaging } \\
\text { Modal- } \\
\text { ity }\end{array}$ & $\begin{array}{l}\text { Prostate } \\
\text { Seg- } \\
\text { men- } \\
\text { tation }\end{array}$ & Features & Classifier & Performance \\
\hline $\begin{array}{l}\text { Puech et } \\
\text { al. [235] }\end{array}$ & $\begin{array}{l}84 \text { Sub- } \\
\text { jects }\end{array}$ & $\begin{array}{l}\text { DCE- } \\
\text { MRI }\end{array}$ & $\begin{array}{l}\text { Manual } \\
\text { ROIs }\end{array}$ & $\begin{array}{l}\text { - Image } \\
\text { Inten- } \\
\text { sity } \\
\text { - Wash- } \\
\text { in Rate } \\
\text { - Wash- } \\
\text { out } \\
\text { Rate } \\
\text { - Time- } \\
\text { to-Peak }\end{array}$ & $\begin{array}{l}\text { Decision } \\
\text { Tree }\end{array}$ & $\begin{array}{r}\text { SEN } \\
(\mathrm{PZ}): \\
1.000 \\
\text { - SPE } \\
(\mathrm{PZ}): \\
0.486 \\
\text { - SEN } \\
(\mathrm{TZ}): \\
1.000 \\
\text { - SPE } \\
(\mathrm{TZ}): \\
0.400\end{array}$ \\
\hline
\end{tabular}


TABLE 5 - continued from previous page

\begin{tabular}{|c|c|c|c|c|c|c|}
\hline Study & Data & $\begin{array}{l}\text { Imaging } \\
\text { Modal- } \\
\text { ity }\end{array}$ & $\begin{array}{l}\text { Prostate } \\
\text { Seg- } \\
\text { men- } \\
\text { tation }\end{array}$ & Features & Classifier & Performance \\
\hline $\begin{array}{l}\text { Puech et } \\
\text { al. [236] }\end{array}$ & $\begin{array}{l}84 \text { Sub- } \\
\text { jects }\end{array}$ & $\begin{array}{l}\text { DCE- } \\
\text { MRI }\end{array}$ & $\begin{array}{l}\text { Manual } \\
\text { ROIs }\end{array}$ & $\begin{array}{l}\text { - Image } \\
\text { Inten- } \\
\text { sity } \\
\text { - Wash- } \\
\text { in Rate } \\
\text { - Wash- } \\
\text { out } \\
\text { Rate } \\
\text { - Tumor } \\
\text { Loca- } \\
\text { tion }\end{array}$ & $\begin{array}{l}\text { Decision } \\
\text { Tree }\end{array}$ & $\begin{array}{r}\text { - SEN } \\
(\mathrm{PZ}): \\
1.00 \\
\text { - } \mathrm{SPE} \\
(\mathrm{PZ}): \\
0.45 \\
\text { - } \mathrm{PPV} \\
(\mathrm{PZ}): \\
0.77 \\
\text { - } \mathrm{SEN} \\
(\mathrm{TZ}): \\
1.00 \\
\text { - } \mathrm{SPE} \\
(\mathrm{TZ}): \\
0.40 \\
\text { - } \mathrm{PPV} \\
(\mathrm{TZ}): \\
0.73\end{array}$ \\
\hline
\end{tabular}


TABLE 5 - continued from previous page

\begin{tabular}{|c|c|c|c|c|c|c|}
\hline Study & Data & $\begin{array}{l}\text { Imaging } \\
\text { Modal- } \\
\text { ity }\end{array}$ & $\begin{array}{l}\text { Prostate } \\
\text { Seg- } \\
\text { men- } \\
\text { tation }\end{array}$ & Features & Classifier & Performance \\
\hline $\begin{array}{l}\text { Sung et } \\
\text { al. [229] }\end{array}$ & $\begin{array}{l}42 \text { Sub- } \\
\text { jects }\end{array}$ & $\begin{array}{l}\text { DCE- } \\
\text { MRI }\end{array}$ & $\begin{array}{l}\text { Manual } \\
\text { ROIs }\end{array}$ & $\begin{array}{l}\text { - Image } \\
\text { Inten- } \\
\text { sity } \\
\text { - } \\
\text { Pharma- } \\
\text { cokinetic } \\
\text { Map } \\
\text { - Wash- } \\
\text { in } \\
\text { - Wash- } \\
\text { out } \\
\text { - Time- } \\
\text { to-peak }\end{array}$ & SVM & $\begin{array}{c}\text { - SEN: } \\
0.77 \\
\text { - SPE: } \\
0.77 \\
\text { - ACC: } \\
0.83\end{array}$ \\
\hline $\begin{array}{l}\text { Vos et al. } \\
\text { [245] }\end{array}$ & $\begin{array}{l}34 \text { Sub- } \\
\text { jects }\end{array}$ & $\begin{array}{l}\text { DCE- } \\
\text { MRI }\end{array}$ & $\begin{array}{l}\text { Manual } \\
\text { ROIs }\end{array}$ & $\begin{array}{l}\text { Pharma- } \\
\text { cokinetic } \\
\text { Map }\end{array}$ & SVM & $\begin{array}{l}-\mathrm{ACC}: \\
0.88\end{array}$ \\
\hline $\begin{array}{l}\text { Vos et al. } \\
\text { [233] }\end{array}$ & $\begin{array}{l}38 \text { Sub- } \\
\text { jects }\end{array}$ & $\begin{array}{l}\text { DCE- } \\
\text { MRI }\end{array}$ & $\begin{array}{l}\text { Intensity } \\
\text { His- } \\
\text { togram and } \\
\text { Hessian- } \\
\text { based Blob } \\
\text { Detection }\end{array}$ & $\begin{array}{l}\text { Pharma- } \\
\text { cokinetic } \\
\text { Map }\end{array}$ & SVM & $\begin{array}{l}-\mathrm{ACC}: \\
0.80\end{array}$ \\
\hline
\end{tabular}


TABLE 5 - continued from previous page

\begin{tabular}{|c|c|c|c|c|c|c|}
\hline Study & Data & $\begin{array}{l}\text { Imaging } \\
\text { Modal- } \\
\text { ity }\end{array}$ & $\begin{array}{l}\text { Prostate } \\
\text { Seg- } \\
\text { men- } \\
\text { tation }\end{array}$ & Features & Classifier & Performance \\
\hline $\begin{array}{l}\text { Viswanath } \\
\text { et } \\
\text { al. [224] }\end{array}$ & $\begin{array}{l}6 \text { Sub- } \\
\text { jects (21 } \\
\text { Images) }\end{array}$ & $\begin{array}{l}\text { DCE- } \\
\text { MRI }\end{array}$ & ASM & $\begin{array}{l}\text { - DCE In- } \\
\text { tensity }\end{array}$ & K-means & $\begin{array}{l}\text { - SEN: } \\
0.4173 \\
\text { - SPE: } \\
0.8454 \\
\text { - ACC: } \\
\\
0.7720\end{array}$ \\
\hline $\begin{array}{l}\text { Firjani et } \\
\text { al. [248] }\end{array}$ & $\begin{array}{l}21 \text { Sub- } \\
\text { jects }\end{array}$ & $\begin{array}{l}\text { DCE- } \\
\text { MRI }\end{array}$ & MAP & $\begin{array}{l}\text { - Wash- } \\
\text { in } \\
\text { - Wash- } \\
\text { out }\end{array}$ & $\mathrm{kNN}$ & $\begin{array}{c}\text { - ACC: } \\
1.00\end{array}$ \\
\hline $\begin{array}{l}\text { Firjani et } \\
\text { al. [67] }\end{array}$ & $\begin{array}{l}28 \text { Sub- } \\
\text { jects (17 } \\
\text { Malig- } \\
\text { nant } \\
\text { and } 11 \\
\text { Benign) }\end{array}$ & DWI & MAP & $\begin{array}{l}\text { - DWI In- } \\
\text { tensity } \\
\text { - ADC } \\
\text { Map }\end{array}$ & $\mathrm{kNN}$ & $\begin{array}{l}\text { - } \mathrm{ACC}: \\
1.00\end{array}$ \\
\hline $\begin{array}{l}\text { Viswanath } \\
\text { et al. [68] }\end{array}$ & $\begin{array}{l}6 \text { Sub- } \\
\text { jects (18 } \\
\text { Images) }\end{array}$ & $\begin{array}{l}\text { T2- } \\
\text { MRI } \\
\text { and } \\
\text { DCE- } \\
\text { MRI }\end{array}$ & ASM & $\begin{array}{l}\text { - T2-MRI } \\
\text { Inten- } \\
\text { sity } \\
\text { - DCE In- } \\
\text { tensity }\end{array}$ & $\begin{array}{l}\text { Random } \\
\text { Forest }\end{array}$ & $\begin{array}{c}\bullet \text { AUC: } \\
0.815\end{array}$ \\
\hline
\end{tabular}


TABLE 5 - continued from previous page

\begin{tabular}{|c|c|c|c|c|c|c|}
\hline Study & Data & $\begin{array}{l}\text { Imaging } \\
\text { Modal- } \\
\text { ity }\end{array}$ & $\begin{array}{l}\text { Prostate } \\
\text { Seg- } \\
\text { men- } \\
\text { tation }\end{array}$ & Features & Classifier & Performance \\
\hline $\begin{array}{l}\text { Chan et } \\
\text { al. [166] }\end{array}$ & $\begin{array}{l}5 \text { Sub- } \\
\text { jects (33 } \\
\text { Images) }\end{array}$ & $\begin{array}{l}\text { T2- } \\
\text { MRI } \\
\text { and } \\
\text { DWI }\end{array}$ & $\begin{array}{l}\text { Manual } \\
\text { ROIs }\end{array}$ & $\begin{array}{l}\text { - } \text { T2 In- } \\
\text { tensity } \\
\text { - T2 In- } \\
\text { tensity } \\
\text { - ADC } \\
\text { Map }\end{array}$ & $\begin{array}{l}\text { LDA and } \\
\text { SVM }\end{array}$ & $\begin{array}{ll}- & \text { AUC } \\
& \text { (LDA): } \\
& 0.839 \\
\pm 0.064 & \\
- & \text { AUC } \\
& (\mathrm{SVM}): \\
& 0.761 \\
\pm 0.043\end{array}$ \\
\hline $\begin{array}{l}\text { Liu et al. } \\
\text { [253] }\end{array}$ & $\begin{array}{l}5 \text { Sub- } \\
\text { jects (33 } \\
\text { Images) }\end{array}$ & $\begin{array}{l}\text { T2- } \\
\text { MRI, } \\
\text { DCE- } \\
\text { MRI } \\
\text { and } \\
\text { DWI }\end{array}$ & None & $\begin{array}{l}\text { T2-MRI } \\
\text { Inten- } \\
\text { sity } \\
\text { • } \\
\text { Pharma- } \\
\text { cokinetic } \\
\text { Map } \\
\text { - ADC } \\
\text { Map }\end{array}$ & $\begin{array}{l}\text { Bayes } \\
\text { classi- } \\
\text { fier and } \\
\text { k-means }\end{array}$ & $\begin{array}{l}\text { - SPE: } \\
0.8958 \\
\text { - SEN: } \\
0.8938 \\
\text { - ACC: } \\
0.8938 \\
\text { - DSC: } \\
0.6222\end{array}$ \\
\hline
\end{tabular}


TABLE 5 - continued from previous page

\begin{tabular}{|c|c|c|c|c|c|c|}
\hline Study & Data & $\begin{array}{l}\text { Imaging } \\
\text { Modal- } \\
\text { ity }\end{array}$ & $\begin{array}{l}\text { Prostate } \\
\text { Seg- } \\
\text { men- } \\
\text { tation }\end{array}$ & Features & Classifier & Performance \\
\hline $\begin{array}{l}\text { Ozer et } \\
\text { al. [226] }\end{array}$ & $\begin{array}{l}20 \text { Sub- } \\
\text { jects }\end{array}$ & $\begin{array}{l}\text { T2- } \\
\text { MRI, } \\
\text { DCE- } \\
\text { MRI } \\
\text { and } \\
\text { DWI }\end{array}$ & None & $\begin{array}{l}\text { - T2-MRI } \\
\text { Inten- } \\
\text { sity } \\
\text { - } \\
\text { Pharma- } \\
\text { cokinetic } \\
\text { Map } \\
\text { - ADC } \\
\text { Map }\end{array}$ & $\begin{array}{l}\text { RVM and } \\
\text { SVM }\end{array}$ & $\begin{array}{l}\text { - } \mathrm{SPE} \\
(\mathrm{RVM}): \\
0.78 \\
\text { - } \mathrm{SEN} \\
(\mathrm{RVM}) \text { : } \\
0.74 \\
\text { - } \mathrm{DSC} \\
\text { (RVM): } \\
0.51 \\
\text { - SPE } \\
\text { (SVM): } \\
0.74 \\
\text { SEN } \\
\text { (SVM): } \\
0.79 \\
\text { DSC } \\
\text { (SVM): } \\
0.52\end{array}$ \\
\hline
\end{tabular}


TABLE 5 - continued from previous page

\begin{tabular}{|c|c|c|c|c|c|c|}
\hline Study & Data & $\begin{array}{l}\text { Imaging } \\
\text { Modal- } \\
\text { ity }\end{array}$ & $\begin{array}{l}\text { Prostate } \\
\text { Seg- } \\
\text { men- } \\
\text { tation }\end{array}$ & Features & Classifier & Performance \\
\hline $\begin{array}{l}\text { Vos et al. } \\
\text { [225] }\end{array}$ & $\begin{array}{l}34 \text { Sub- } \\
\text { jects }\end{array}$ & $\begin{array}{l}\text { T2- } \\
\text { MRI } \\
\text { and } \\
\text { DCE- } \\
\text { MRI }\end{array}$ & Manual & $\begin{array}{l}\text { - } \text { T2-MRI } \\
\text { Inten- } \\
\text { sity } \\
\text { • T2-MRI } \\
\text { Relax- } \\
\text { ation } \\
\text { Time } \\
\text { - } \\
\text { Pharma- } \\
\text { cokinetic } \\
\text { Map }\end{array}$ & SVM & $\begin{array}{c}-\mathrm{ACC}: \\
0.89\end{array}$ \\
\hline $\begin{array}{l}\text { Litjens et } \\
\text { al. [69] }\end{array}$ & $\begin{array}{l}188 \\
\text { Subjects }\end{array}$ & $\begin{array}{l}\text { T2- } \\
\text { MRI, } \\
\text { DCE- } \\
\text { MRI } \\
\text { and } \\
\text { DWI }\end{array}$ & $\begin{array}{l}\text { Probabilistic } \\
\text { Model }\end{array}$ & $\begin{array}{l}\text { T2-MRI } \\
\text { Inten- } \\
\text { sity } \\
\text { • } \\
\text { Pharma- } \\
\text { cokinetic } \\
\text { Map } \\
\text { - ADC } \\
\text { Map }\end{array}$ & SVM & $\begin{array}{r}\text { - SEN: } \\
0.834\end{array}$ \\
\hline
\end{tabular}


TABLE 5 - continued from previous page

\begin{tabular}{|c|c|c|c|c|c|c|}
\hline Study & Data & $\begin{array}{l}\text { Imaging } \\
\text { Modal- } \\
\text { ity }\end{array}$ & $\begin{array}{l}\text { Prostate } \\
\text { Seg- } \\
\text { men- } \\
\text { tation }\end{array}$ & Features & Classifier & Performance \\
\hline $\begin{array}{l}\text { Niaf et } \\
\text { al. [71] }\end{array}$ & $\begin{array}{l}30 \text { Sub- } \\
\text { jects }\end{array}$ & $\begin{array}{l}\text { T2- } \\
\text { MRI, } \\
\text { DCE- } \\
\text { MRI } \\
\text { and } \\
\text { DWI }\end{array}$ & $\begin{array}{l}\text { Manual } \\
\text { ROIs }\end{array}$ & $\begin{array}{l}\text { - T2-MRI } \\
\text { Inten- } \\
\text { sity } \\
\text { - T2-MRI } \\
\text { Inten- } \\
\text { sity } \\
\text { Gradi- } \\
\text { ent } \\
\text { - T2-MRI } \\
\text { Sobel } \\
\text { filter } \\
\text { - DCE In- } \\
\text { tensity } \\
\text { - DCE } \\
\text { AUGC } \\
\text { - Wash- } \\
\text { in } \\
\text { - ADC } \\
\text { Map } \\
\text { Sobel } \\
\text { filter }\end{array}$ & SVM & $\begin{array}{l}\text { - AUC: } \\
0.89\end{array}$ \\
\hline
\end{tabular}


TABLE 5 - continued from previous page

\begin{tabular}{|c|c|c|c|c|c|c|}
\hline Study & Data & $\begin{array}{l}\text { Imaging } \\
\text { Modal- } \\
\text { ity }\end{array}$ & $\begin{array}{l}\text { Prostate } \\
\text { Seg- } \\
\text { men- } \\
\text { tation }\end{array}$ & Features & Classifier & Performance \\
\hline $\begin{array}{l}\text { Shah et } \\
\text { al. [74] }\end{array}$ & $\begin{array}{l}24 \text { Sub- } \\
\text { jects }\end{array}$ & $\begin{array}{l}\text { T2- } \\
\text { MRI, } \\
\text { DCE- } \\
\text { MRI } \\
\text { and } \\
\text { DWI }\end{array}$ & K-means & $\begin{array}{l}\text { T2-MRI } \\
\text { Inten- } \\
\text { sity } \\
\text { - } \\
\text { Pharma- } \\
\text { cokinetic } \\
\text { Map } \\
\text { - ADC } \\
\text { Map }\end{array}$ & SVM & $\begin{array}{c}\text { - F-M: } \\
0.89 \\
\text { - SEN: } \\
0.90 \\
\text { - SPE: } \\
0.90\end{array}$ \\
\hline $\begin{array}{l}\text { Hambrock } \\
\text { et al. [72] }\end{array}$ & $\begin{array}{l}34 \text { Sub- } \\
\text { jects }\end{array}$ & $\begin{array}{l}\text { T2- } \\
\text { MRI, } \\
\text { DCE- } \\
\text { MRI } \\
\text { and } \\
\text { DWI }\end{array}$ & $\begin{array}{l}\text { Manual } \\
\text { ROIs }\end{array}$ & $\begin{array}{l}\text { - T2 } \\
\text { Wash- } \\
\text { out } \\
\text { - ADC } \\
\text { Map }\end{array}$ & LDA & $\begin{array}{l}-\mathrm{AUC}: \\
0.90\end{array}$ \\
\hline $\begin{array}{l}\text { Vos et al. } \\
{[70]}\end{array}$ & $\begin{array}{l}29 \text { Sub- } \\
\text { jects }\end{array}$ & $\begin{array}{l}\text { T2- } \\
\text { MRI, } \\
\text { DCE- } \\
\text { MRI } \\
\text { and } \\
\text { DWI }\end{array}$ & $\begin{array}{l}\text { Probabilistic } \\
\text { Model }\end{array}$ & $\begin{array}{l}\text { T2 In- } \\
\text { tensity } \\
\text { - } \\
\text { Pharma- } \\
\text { cokinetic } \\
\text { Map } \\
\text { - ADC } \\
\text { Map }\end{array}$ & SVM & $\begin{array}{l}\bullet \text { ACC: } \\
0.833 \\
\pm 0.052\end{array}$ \\
\hline
\end{tabular}


TABLE 5 - continued from previous page

\begin{tabular}{|c|c|c|c|c|c|c|}
\hline Study & Data & $\begin{array}{l}\text { Imaging } \\
\text { Modal- } \\
\text { ity }\end{array}$ & $\begin{array}{l}\text { Prostate } \\
\text { Seg- } \\
\text { men- } \\
\text { tation }\end{array}$ & Features & Classifier & Performance \\
\hline $\begin{array}{l}\text { Peng et } \\
\text { al. [73] }\end{array}$ & $\begin{array}{l}48 \text { Sub- } \\
\text { jects }\end{array}$ & $\begin{array}{l}\text { T2- } \\
\text { MRI, } \\
\text { DCE- } \\
\text { MRI } \\
\text { and } \\
\text { DWI }\end{array}$ & $\begin{array}{l}\text { Manual } \\
\text { ROIs }\end{array}$ & $\begin{array}{l}\text { - T2-MRI } \\
\text { Inten- } \\
\text { sity } \\
\text { - ADC } \\
\text { Map }\end{array}$ & LDA & $\begin{array}{c}\text { - } \text { AUC: } \\
0.95 \\
\pm 0.02 \\
\text { - SEN: } \\
0.82 \\
\text { - SPE: } \\
0.95\end{array}$ \\
\hline $\begin{array}{l}{ }^{*} \mathrm{ACC} \text { de } \\
{ }^{*} \mathrm{ROC} \text { der } \\
{ }^{*} \mathrm{AUC} \text { de } \\
{ }^{*} \mathrm{DSC} \text { der } \\
{ }^{*} \mathrm{~F}-\mathrm{M} \text { den } \\
{ }^{*} \mathrm{PPV} \text { den } \\
{ }^{*} \mathrm{SEN} \text { den } \\
{ }^{*} \mathrm{SPE} \text { den }\end{array}$ & $\begin{array}{l}\text { tes accura } \\
\text { tes receiv } \\
\text { tes area u } \\
\text { tes Dice's } \\
\text { tes F-Meas } \\
\text { tes positiv } \\
\text { tes sensiti } \\
\text { es specific }\end{array}$ & $\begin{array}{l}\text { y. } \\
\text { r operating } \\
\text { dder the RO } \\
\text { imilarity co } \\
\text { Are. } \\
\text { predictive } \\
\text { ity. } \\
\text { ty. }\end{array}$ & $\begin{array}{l}\text { characteri } \\
\text { C curve. } \\
\text { efficient. } \\
\text { value. }\end{array}$ & & & \\
\hline
\end{tabular}

\section{Discussion}

Several in-vitro and in-vivo diagnostics technologies have been investigated for the diagnosis of prostate cancer. While in-vitro techniques are commonly used clinically, they have several limitations concerning their accuracy and the invasive nature of most methods. Recent trends investigate developing in-vivo non-invasive image-based CAD-systems to provide reliable diagnosis of prostate cancer in its earliest stage, which would eliminate the need for collecting physical patient samples, improve the effectiveness of treatment, and increase the patient's chance of survival. This study covers both in-vitro and in-vivo techniques for prostate cancer 
diagnostics. In this section, the potentials and limitations of the current techniques, the challenges they face, and the recent trends for prostate cancer diagnosis are presented.

\section{Potentials and limitations of the current prostate diagnostic techniques}

Current prostate cancer diagnostic techniques have the following potentials and limitations:

- DRE examination is invasive, inexpensive, and easy to perform. However, it is subject to a physicians subjective analysis and can only detect sufficiently large tumors.

- Current in-vitro studies that are based on urine biomarkers are non-invasive and relatively inexpensive. However, they can have high false positive and false negative rates $[50,51]$.

- Current in-vitro studies that are based on blood biomarkers are relatively inexpensive. In addition, early diagnosis of prostate cancer is usually per-

formed using blood-based PSA analysis [4]. However, they are invasive, can lead to bleeding, and can also have high false positive and false negative rates $[50,51]$.

- Needle biopsies remain the gold standard for diagnosis of prostate cancer, but are the last resort because of their invasive nature, high cost, and potential morbidity rate. In addition, Gleason scores of biopsy-collected tissue samples are dependent on the observer analyzing the sample [53]. Moreover, the relatively small needle biopsy samples have a higher possibility of producing false positive diagnoses.

- Imaging-based CAD systems for prostate cancer depend on analyzing TRUS and MRI images. They are highly non-invasive and can be used to provide 
early diagnosis, improve patent's treatment, and assist in image-guided surgeries. However, several challenges still exist to continue improving these techniques in terms of automation and accuracy.

\section{Research Challenges}

Several research challenges face current $C A D$ techniques for prostate cancer. These challenges include:

- Developing methods for accurate automated segmentation of the prostate is still challenging due to (i) the noisy nature of MRI and TRUS images, (ii) the proximity and similarity in intensity of surrounding non-prostate tissues, such as the bladder, and (iii) the varying shape and size of the prostate between subjects.

- Developing CAD systems based on multi-modalities (e.g. TRUS, T1-MRI, T2-MRI, DCE-MRI and DWI-MRI) are promising due to the increased set of diagnostic features. However, they are challenging due to the different resolutions of the varying image modalities and the inter-slice variability between the obtained images. To develop such systems, researchers face the following challenges:

- Developing efficient registration algorithms to align the imaging modalities is very challenging.

- Developing segmentations algorithm that work for the wide variety of imaging modalities is very challenging.

- Determining the optimal set a features that accurately discriminate between the benign and malignant classes is challenging. 


\section{Trends}

Several trends have become apparent in the development of the segmentation, feature extraction and classification components of CAD systems:

- Recent trends for prostate segmentation develop more accurate shape models to segment the noisy MRI images.

- In recent years, DCE-MRI has had considerable success in detecting and locating prostate cancer. However, intravenous administration of a contrast agent can potentially harm a patient's kidneys [65]. In addition, injecting and waiting for the contrast agent to settle in the prostate increases the time required to scan the patient. Diffusion weighted imaging (DWI) [66] and diffusion tensor imaging are new alternative MRI techniques that avoid using contrast agents and have shown promising results in detecting the prostate cancer.

- Recent trends for developing CAD systems have increasingly combined the features from several modalities for classification (e.g. multi-parametric MRI). This allows for a larger set of possible features to be selected from when constructing discriminative feature vectors, thereby increasing the quality of a system's classification.

- Recent trends integrate both in-vitro biomarkers with imaging biomarkers to increase the diagnostic efficiency.

\section{Conclusion}

Designing efficient in-vitro and in-vivo techniques for detecting prostate cancer is crucial for the management of prostate cancer progress in patients. When there is an optimal opportunity to intervene using existing clinical strategies (i.e., chemo- or radiation-therapy), reliable and early detection of prostate cancer for 
an individual patient in the earliest stages may represent an important advance in the personalized management of this condition. In recent years, several in-vitro and in-vivo technologies have been proposed for the detection and characterization of prostate cancer. This chapter presented a comprehensive overview of these systems, covering in-vitro biomarker tests and needle biopsies, as well as in-vivo non-invasive TRUS-based and MRI-based CAD systems. Current approaches that were developed for each stage of prostate cancer CAD systems, with emphasis on their strengths and limitations, were also addressed. An accurate diagnostic CAD system could decrease the deaths resulting from prostate cancer due to earlier disease diagnosis. Additionally, the challenges and new trends for improving prostate cancer diagnosis have been discussed. Subsequently, there is a compelling need for researchers to make significant strides in advancing the state of the art in prostate cancer diagnostic methods to improve their clinical viability. 


\section{CHAPTER II}

\section{A NOVEL NMF GUIDED LEVEL-SET FOR DWI PROSTATE SEGMENTATION}

In this chapter, a three dimensional (3D) level-set-based framework for the segmentation of the prostate from diffusion weighted imaging (DWI) magnetic resonance imaging (MRI) is proposed. The level-set deformable model is guided by a novel stochastic speed function that is derived using nonnegative matrix factorization (NMF), which extracts meaningful features from a high-dimensional feature space. The NMF attributes are calculated using information from the MRI intensity, a probabilistic shape model, and the spatial interactions between prostate voxels. The shape model is constructed using a set of training prostate volumes and then updated during the segmentation process using an appearance based method that takes into account both a voxel's location and its intensity value. The spatial interactions are modeled using a second order pairwise 3D Markov-Gibbs random field (MGRF). Experiments on in-vivo DWI-MRI prostate data for 9 subjects show that using this information along with NMF-based feature fusion to guide the level-set increases accuracy compared with previously proposed methods using two metrics, the dice similarity coefficient (DSC) and Hausdorf distance (HD). The proposed method achieved an average DSC of $0.870 \pm 0.03$ and an average HD of $5.72 \pm 2.35 \mathrm{~mm}^{3}$ compared to an average DSC of $0.833 \pm 0.07$ and an average HD of $6.74 \pm 2.04$ for a maximum a posteriori (MAP)-based level-set and an average DSC of $0.810 \pm 0.05$ and an average HD of $9.07 \pm 1.64$ for a level-set driven only by intensity and shape information. 


\section{A Introduction}

In order to perform image-based analysis of the prostate (e.g. for the purpose of prostate cancer detection), the prostate must first be located. However, this can be challenging due to image noise, inter-patient anatomical differences, and the similar intensities of the prostate and surrounding tissues (e.g. the bladder). Several methods have been proposed to overcome these challenges as discussed in the previous chapter. In this chapter, a novel nonnegative matrix factorization (NMF) driven level-set algorithm is proposed for DWI prostate segmentation.

NMF is a method for extracting meaningful features from data sets to perform clustering [255]. This is done by calculating a weight matrix $W$ that transform a vector from the input space into a new feature space ( $H$-space) through factorizing the input matrix $A$ so that $A \approx W H$. NMF has been applied to various data analysis problems such as document clustering [256] and facial recognition [257]. In addition, it has been used in a few segmentation systems. This includes Xie et al. [258] who used NMF to segment the spinal cord, corpus callosum, and hippocampus regions of rats from diffusion tensor images (DTI) by k-means clustering the column vectors of the produced $H$ matrix. Also, Sandler et al. [259] proposed using NMF to factorize intensity histogram data for generic image segmentation. While applying NMF to image segmentation appears promising, further research is required to verify its usefulness.

Level-set segmentation is a geometric deformable model technique that is commonly used in object segmentation. It has been applied to segment several organs in the human body (e.g. the kidneys [97] and the heart [96]). In addition, it has also been used to segment the prostate from DWI data with some success. In [178], Liu et al. proposed a 2D level-set guided by intensity and shape information for DWI prostate segmentation. Also, Liu et al. developed a 3D level-set method that was also guided by intensity and shape information [179]. In this chapter, a novel DWI prostate segmentation framework is proposed that utilizes NMF to 


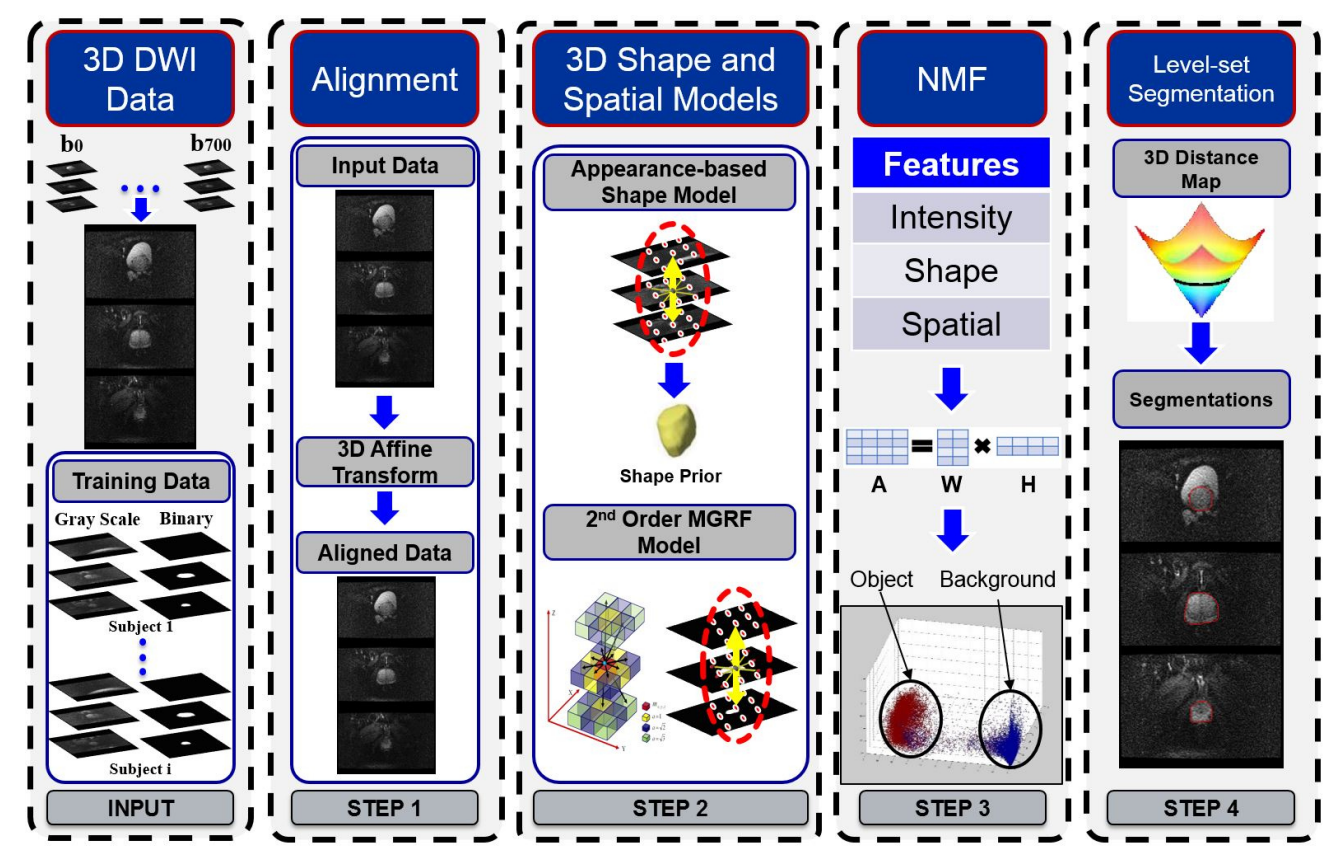

Figure 6. A diagram of the proposed segmentation framework.

acquire better features for guiding the evolution of a 3D level-set.

\section{B Methods}

In this chapter, a novel DWI prostate segmentation framework (Fig. 6) is proposed. It utilizes an NMF-based feature fusion approach that incorporates three features, namely DWI intensity, shape, and spatial information. The features generated by performing NMF-based feature fusion are then used to guide the evolution of a 3D level-set deformable model to extract the prostate from DWI data. The definition of this level-set is given below. The evolving surface of the level-set at any time instant $t$ is represented by the zero level, $\phi_{n+1}(x, y, z)=0$, of an implicit level-set function, namely a distance map of the signed minimum Euclidean distance from each voxel to the surface. This formulation results in points inside the surface having negative (or positive) values and voxels outside the surface hav- 
ing positive (or negative) values, respectively. Mathematically, the evolution of the level-set is defined by [100]:

$$
\phi_{n+1}(x, y, z)=\phi_{n}(x, y, z)-\tau V_{n}(x, y, z)\left|\nabla \phi_{n}(x, y, z)\right|
$$

where $t$ is the discrete time instant $t=n \tau$ taken with a step $\tau, \tau>0$ and $\nabla=$ $\left[\frac{\partial}{\partial x}, \frac{\partial}{\partial y}, \frac{\partial}{\partial z}\right]$ is the differential operator. This evolution is guided by the speed function $V_{n}(x, y, z)$ [260].

Previous speed functions that use image intensities, object edges, and gradient vector flow have had difficulty segmenting noisy images and those with poor object-background contrast. More effective speed functions have been developed by using shape priors to incorporate shape information of the object of interest. However, this has not completely overcome image inhomogeneities (e.g. large image noise and discontinuous object boundaries). In order to more accurately segment the prostate from DWI data, we propose a speed function that takes into account the 3D appearance, shape, and spatial features of the DWI data. These features are combined using an NMF-based fusion method to provide the voxelwise guidance of the deformable model.

\section{3D Appearance, Shape, and Spatial Features}

Basic Notation: Let $\mathbf{Q}=\{0, \ldots, Q-1\}$ and $\mathbf{L}=\{0,1\}$ be the set of $Q$ integer gray levels and a set of object (1) and background (0) labels, respectively. Also, let a $3 \mathrm{D}$ arithmetic lattice $\mathbf{R}=\{(x, y, z): 0 \leq x \leq X-1 ; 0 \leq y \leq Y-1 ; 0 \leq z \leq$ $Z-1\}$ support the grayscale DWI data $\mathbf{g}: \mathbf{R} \rightarrow \mathbf{Q}$ and their binary region maps $\mathbf{m}: \mathbf{R} \rightarrow \mathbf{L}$. Each voxel $(x, y, z)$ is associated with its neighbors, $\{(x+\xi, y+\eta, z+\zeta):$ $(x+\xi, y+\eta, z+\zeta) \in \mathbf{R} ;(\xi, \eta, \zeta) \in \mathbf{N}\}$ where $\mathbf{N}$ was defined by $\xi \in\{-1,0,1\}$, $\eta \in\{-1,0,1\}$, and $\zeta \in\{-1,0,1\}$ (Fig. 7).

Appearance-Based Shape Model: Most prostates have a similar near-ellipsoidal shape [6]. As a result, the inclusion of a shape prior can significantly improve the 


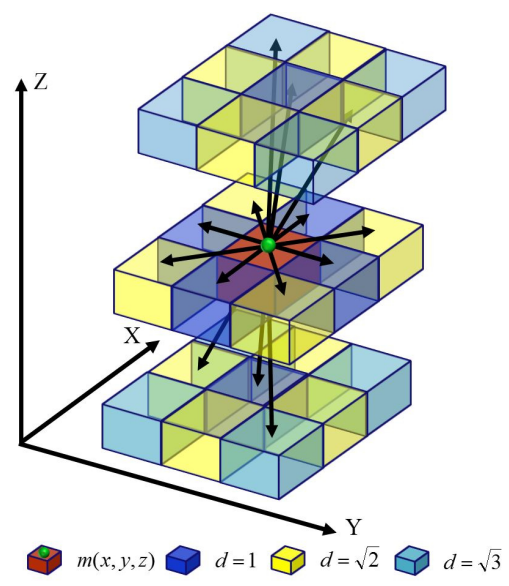

Figure 7. Illustration of a voxel's neighborhood.

segmentation accuracy. In the proposed framework, an appearance-based shape model is built that takes into account not only a voxel's location, but also its intensity information. A shape database was constructed by co-aligning training data sets using a 3D affine transformation with 12 degrees of freedom ( 3 for the 3D translation, 3 for the 3D rotation, 3 for the 3D scaling, and 3 for the 3D shearing) and maximizing mutual information (MI) [261]. A shape prior is a spatially variant independent random field of region labels for the co-aligned data. Mathematically, this is defined as:

$$
P_{\text {shape }}(\mathbf{m})=\prod_{(x, y, z) \in \mathrm{R}} P_{\text {shape: } x, y, z}\left(m_{x, y, z}\right)
$$

where $P_{\text {shape: } x, y, z}(l)$ is the voxel-wise empirical probability for label $l \in \mathbf{L}$. For each input DWI volume to be segmented, the shape prior is constructed by a process guided by the visual appearance features of the DWI data. The appearance-based shape prior is then estimated using the method summarized in Algorithm 1.

Spatial Voxel Interaction Model: In addition to the prostate shape prior, analyzing the interactions of a voxel and its neighbors can improve segmentation $[67,99]$. In order to model these interactions, a second-order 3D MGRF model [262] 
Algorithm 1 Algorithm for Calculating an Appearance-based Shape Model Calculate the value of the shape prior probability at each voxel using the following steps:

1. Transform each test subject voxel to the shape database domain using the calculated 3D affine transformation matrix (T).

2. Initialize an $N_{1 i} \times N_{2 i} \times N_{3 i}$ search space centered at the voxel.

3. Find voxels inside the search space with corresponding gray levels to the center voxel in all training data sets.

4. If no corresponding voxels are found, increase the search space size and repeat the previous step.

5. Calculate the label probabilities for each voxel based on the relative occurrence of each label in the search results.

is used. The MGRF model of the region map $\mathbf{m}$ is defined as:

$$
P_{\text {spatial }}(\mathbf{m})=\frac{1}{Z_{\mathbf{N}}} \exp \sum_{(x, y, z) \in \mathbf{R}} \sum_{(\varepsilon, \nu, \zeta) \in \mathbf{N}} V_{e q}\left(m_{x, y, z}, m_{x+\varepsilon, y+\nu, z+\zeta}\right)
$$

where $V_{e q}\left(m_{x, y, z}, m_{x+\varepsilon, y+\nu, z+\zeta}\right)$ is the Gibbs potential and $Z_{N}$ is the normalization factor which can be approximated as [263]:

$$
Z_{N} \approx \exp \sum_{(x, y, z) \in \mathbf{R}} \sum_{(\varepsilon, \nu, \zeta) \in \mathbf{N}} \sum_{l \in \mathbf{L}} V_{e q}\left(l, m_{x+\varepsilon, y+\nu, z+\zeta}\right)
$$

The MGRF used can be viewed as a 3D extension of the auto-binomial, or Potts, model with the exception that the Gibbs potential is estimated analytically. The maximum likelihood estimate of the potential is given as [189]:

$$
V_{e q}=2\left(f_{e q}(\mathbf{m})-\frac{1}{2}\right)
$$

where $f_{e q}(\mathbf{m})$ is the relative frequency of equal (eq) labels in the voxel pairs $((x, y, z),(x+$ $\xi, y+\eta, z+\zeta))$. 


\section{NMF-based Feature Fusion}

NMF is a method for extracting meaningful features from data sets for representing different categories in the data [255]. This is done by calculating a weight matrix $W$ that transforms a vector from the input space into a new feature space ( $H$-space) through factorizing the input matrix $A$ so that $A \approx W H$. NMF has been applied to various data analysis problems such as document clustering [256] and facial recognition [257]. In addition, it has been used in a few segmentation systems. This includes Xie et al. [258] who used NMF to segment the spinal cord, corpus callosum, and hippocampus regions of rats from diffusion tensor imaging (DTI) by k-means clustering of the column vectors of the produced $H$ matrix. Also, Sandler et al. [259] proposed using NMF to factorize intensity histogram data for generic image segmentation.

In this chapter, NMF is proposed to find the weights for each feature in order to create a feature space where object and background classes are better separated, dimensionality is reduced, and information from the training data set is encoded. NMF factorizes a $k$ by $n$ input matrix $A$ into a $k$ by $r$ weight matrix $W$, which contains the basis vectors of the new space as columns, and an $r$ by $n$ output matrix $H$ where $k$ is the dimensionality of the input column vectors, $n$ is the number of input and output column vectors, and $r$ is the dimensionality of the output column vectors [255]. Mathematically, this is defined as:

$$
A \approx W H
$$

$W$ and $H$ are calculated by minimizing the Euclidean distance between $A$ and $W H$ with the constraint that $W$ and $H$ contain only non-negative values. This results in the constrained optimization problem: 


$$
\begin{array}{ll}
\underset{W, H}{\operatorname{minimize}} & \frac{1}{2}\|A-W H\|^{2} \\
\text { subject to } & W, H \geq 0
\end{array}
$$

In the literature, several methods have been used to optimize this function. The most prominent methods have been multiplicative gradient descent, alternating least square (ALS), and projected gradient descent (PGD) [264]. In this chapter, the multiplicative method [265] is used because of its ease of implementation. This method iteratively updates $W$ and $H$ until convergence using the following rules:

$$
\begin{gathered}
H_{\alpha \beta} \leftarrow H_{\alpha \beta} \frac{\left(W^{T} A\right)_{\alpha \beta}}{\left(W^{T} W H\right)_{\alpha \beta}} \\
W_{\gamma \alpha} \leftarrow W_{\gamma \alpha} \frac{\left(A H^{T}\right)_{\gamma \alpha}}{\left(W H H^{T}\right)_{\gamma \alpha}}
\end{gathered}
$$

where $\alpha: 1 \rightarrow r, \beta: 1 \rightarrow n$, and $\gamma: 1 \rightarrow k$.

In the proposed framework, NMF is performed on a matrix that has a $k^{\text {th }} \mathrm{di}^{-}$ mensional, one dimension for each calculated feature, column vector for each voxel $(x, y, z)$ in the training volumes. The input features are the intensity values of the voxe $(x, y, z)$ and its neighbors, the spatial interactions between voxel $(x, y, z)$ and its neighbors, and the value of the shape prior at $(x, y, z)$. The resulting $W$ is used as the basis vectors to transform new feature vectors into the new $r$-dimensional space ( $H$-space). The resulting $H$ is used to find the $r$-dimensional centroids corresponding to the object and background classes, $C_{\text {object }}$ and $C_{\text {background, }}$, respectively. For each voxel in a testing volume, a $k^{t h}$ dimensional feature vector was calculated. This resulted in a $k$ by $n$ feature matrix $B$ where $n$ is the number of voxels in the volume. The new $r$ dimensional vectors corresponding to the input voxels are calculated by multiplying $B$ by the pseudo-inverse of $W$, which can be replaced by $W^{T}$ assuming orthogonality of the columns of $W$ [266]. Mathematically, this is described as: 


$$
H_{B}=W^{T} B
$$

\section{Estimation of the Stochastic Speed Function}

In this chapter, a novel speed function to control the evolution of the levelset deformable model is proposed. This speed function is derived using the NMFbased fusion of DWI features, $H_{B: x, y, z}$ for voxel $(x, y, z)$. The proposed speed function $V_{n}(x, y, z)$ is defined as $V_{n}(x, y, z)=\kappa \vartheta(x, y, z)$, where $\kappa$ is the curvature and $\vartheta(x, y, z)$ is defined as:

$$
\vartheta(x, y, z)=\left\{\begin{array}{cl}
-E_{1: x, y, z} & \text { if } E_{1: x, y, z}>E_{0: x, y, z} \\
E_{0: x, y, z} & \text { otherwise }
\end{array}\right.
$$

Here, $E_{1: x, y, z}=\frac{P_{n m f: x, y, z}(1)+P_{\text {shape: } x, y, z}(1)+P_{\text {spatial: } x, y, z}(1)}{3}$ where $P_{\text {shape: } x, y, z}(1)$ is the object shape prior probability and $P_{\text {spatial:x,y,z }}(1)$ is the object MGRF model probability (Eq. 3). Similarly, $E_{0: x, y, z}=\frac{P_{n m f: x, y, z}(0)+P_{\text {shape: } x, y, z}(0)+P_{\text {spatial: } x, y, z}(0)}{3}$ where $P_{\text {shape: } x, y, z}(0)$ is the background shape prior probability and $P_{\text {spatial: } x, y, z}(0)$ is the background MGRF model probability (Eq. 3). $P_{n m f: x, y, z}(1)$ and $P_{n m f: x, y, z}(0)$ are defined as:

$$
\begin{aligned}
P_{n m f: x, y, z}(1) & =\frac{\frac{1}{d_{1}\left(H_{B: x, y, z}\right)}}{\frac{1}{d_{1}\left(H_{B: x, y, z}\right)}+\frac{1}{d_{1}\left(H_{B: x, y, z}\right)}} \\
P_{n m f: x, y, z}(0) & =\frac{\frac{1}{d_{0}\left(H_{B: x, y, z}\right)}}{\frac{1}{d_{0}\left(H_{B: x, y, z}\right)}+\frac{1}{d_{0}\left(H_{B: x, y, z}\right)}}
\end{aligned}
$$

where $d_{1}(x, y, z)$ and $d_{0}(x, y, z)$ are the Euclidean distances from the $r$-dimensional vector in $H_{B}$ corresponding to the input voxel $(x, y, z)$ to the centroids of the object and background classes, $C_{1}$ and $C_{0}$, respectively, in $H$-space. The overall segmentation framework is summarized by Algorithm 2. 
Algorithm 2 Proposed Algorithm for DWI Prostate Segmentation

Segment the prostate from a DWI volume by:

1. Align the input DWI volume with the training database using the MI-based affine transformation.

2. Calculate the appearance-based shape prior using Algorithm 1.

3. Calculate the 3D pairwise voxel interactions (Eq. 3).

4. Perform NMF-based feature fusion.

5. Calculate the probabilities that each voxel is object or background using the NMF-based features (Eq. 12 and Eq. 13).

6. Use these probabilities to guide the evolution of a level-set to segment the prostate (Eq. 11).

\section{Performance Metrics}

The performance of the proposed segmentation framework was evaluated using two metrics: (1) Dice similarity coefficient (DSC) and (2) Hausdorf distance (HD). These metrics are detailed below.

\section{Dice Similarity Coefficient (DSC)}

Many segmentation and classification metrics are based on the determination of true positive (TP), false positive (FP), true negative (TN), and false negative (FN) values (see Fig. 8). The TP is the number of correctly positively labeled sam-

ples; the FP is the number of incorrectly positively labeled samples; the TN is the number of correctly negatively labeled samples; and the FN is the number of incorrectly negatively labeled samples. These values can be used to calculate the DSC given by:

$$
D S C=\frac{2 T P}{2 T P+F P+F N}
$$


The value of the DSC ranges from 0 to 1 , where 0 means that there is no similarity and 1 means that there is perfect similarity.

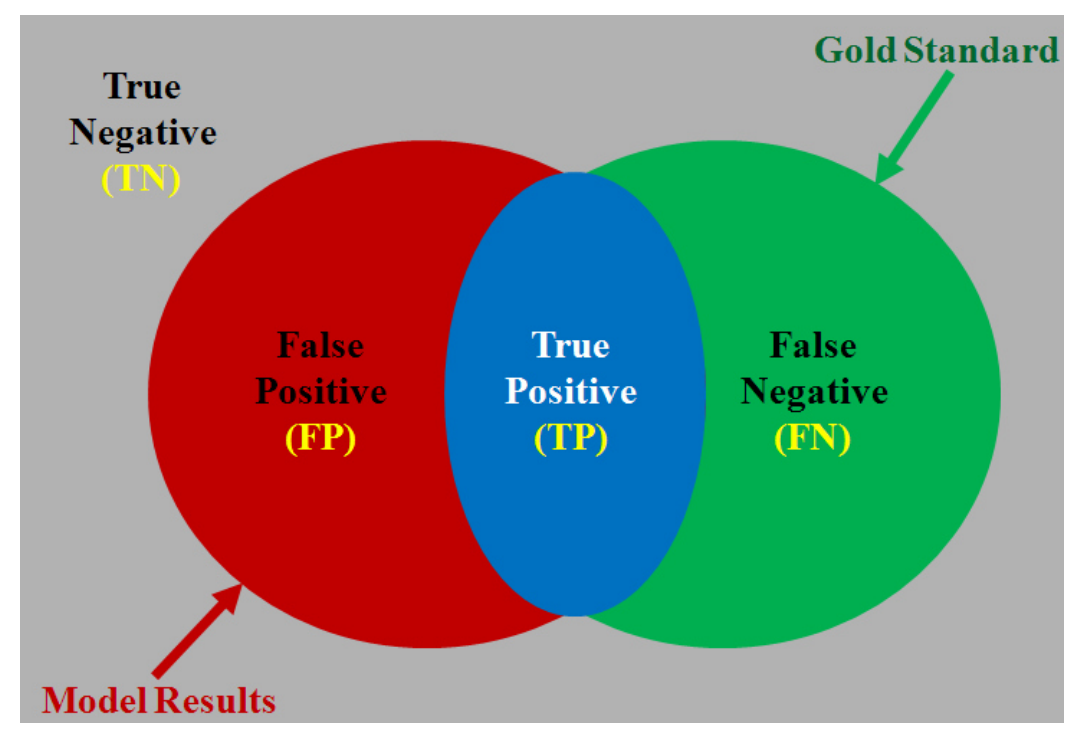

Figure 8. Diagram illustrating the meaning of TP, FP, TN and FP.

\section{Hausdorf Distance (HD)}

Distance measures are another type of performance metric used for evaluating segmentation methods. The Euclidean distance is often utilized, but another common measure is the HD (See Fig. 9). The HD from a set $\mathbf{A}_{1}$ to a set $\mathbf{A}_{\mathbf{2}}$ is defined as the maximum distance of the set $\mathbf{A}_{1}$ to the nearest point in the set $\mathbf{A}_{2}$ [267]:

$$
H D\left(\mathbf{A}_{\mathbf{1}}, \mathbf{A}_{\mathbf{2}}\right)=\max _{a_{\mathbf{1}} \in \mathbf{A}_{\mathbf{1}}}\left\{\min _{a_{\mathbf{2}} \in \mathbf{A}_{\mathbf{2}}}\left\{d\left(a_{1}, a_{2}\right)\right\}\right\}
$$

where $a_{1}$ and $a_{2}$ are points of sets $\mathbf{A}_{\mathbf{1}}$ and $\mathbf{A}_{\mathbf{2}}$, respectively, and $d\left(a_{1}, a_{2}\right)$ is Euclidean distance between these points. The bidirectional Hausdorff distance, denoted by $H D_{B i}(\mathbf{G T}, \mathbf{S R})$, between the segmented region (SR) and its ground truth $(\mathrm{GT})$ is defined as: 


$$
H D_{B i}(\mathbf{G T}, \mathbf{S R})=\max \{H D(\mathbf{G T}, \mathbf{S R}), H D(\mathbf{S R}, \mathbf{G T})\}
$$

The smaller the distance, the better the segmentation. The ideal case with perfect segmentation is when the bidirectional Hausdorff distance is equal to 0 .



Figure 9. Diagram illustrating the 2D HD of boundaries $A_{1}$ and $A_{2}$ for points $a_{1}$ and $a_{2}$.

\section{Experimental Results}

\section{Medical Images}

The proposed system was tested on 9 subjects, each with DWI volumes acquired at using a scanner (SIGNA Horizon, General Electric Medical Systems, Milwaukee, WI) with the following parameters: TE: 84:6 ms; TR: $8.000 \mathrm{~ms}$; FOV 32 $\mathrm{cm}$; slice thickness $3 \mathrm{~mm}$; inter-slice gap $0 \mathrm{~mm}$; and two excitations. The data was with $b$-values ranging of $0,100,200,300,400,500,600$, and 700 using a voxel size of $1.25 \times 1.25 \times 3.00 \mathrm{~mm}^{3}$. The ground truth segmentations used in training and in verifying the segmentation results were manually created by an MR expert for each subject. 
$S_{3}$
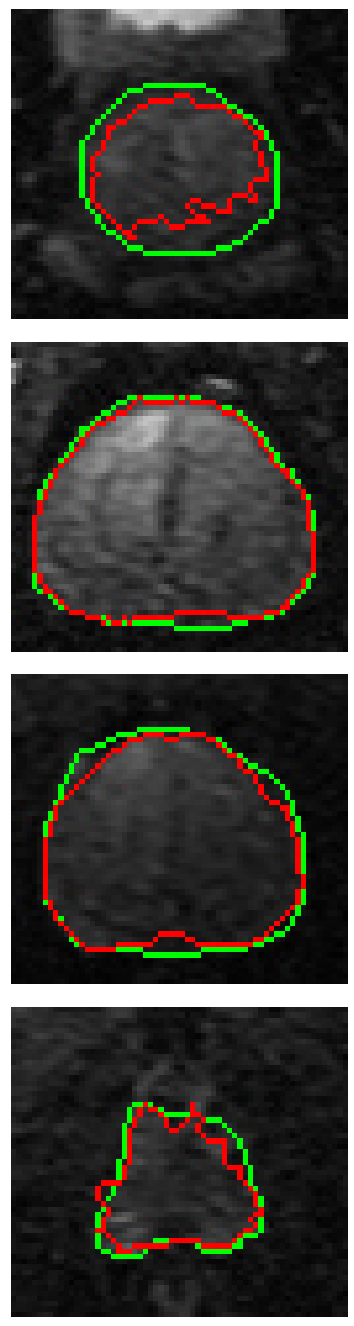

$S_{4}$
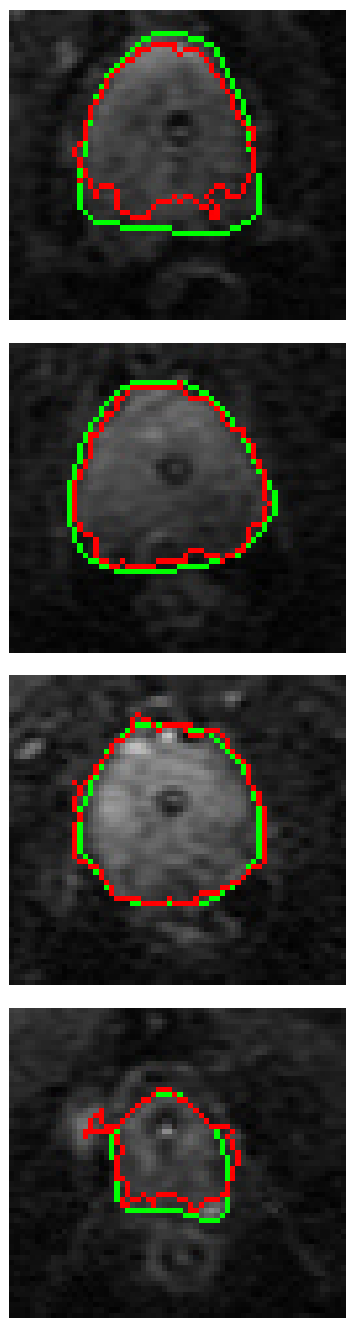

$S_{8}$
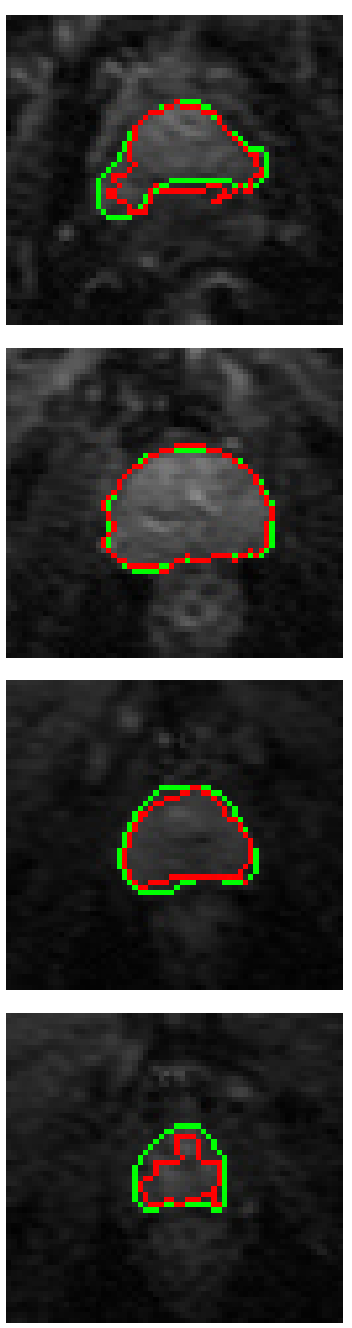

$S_{9}$
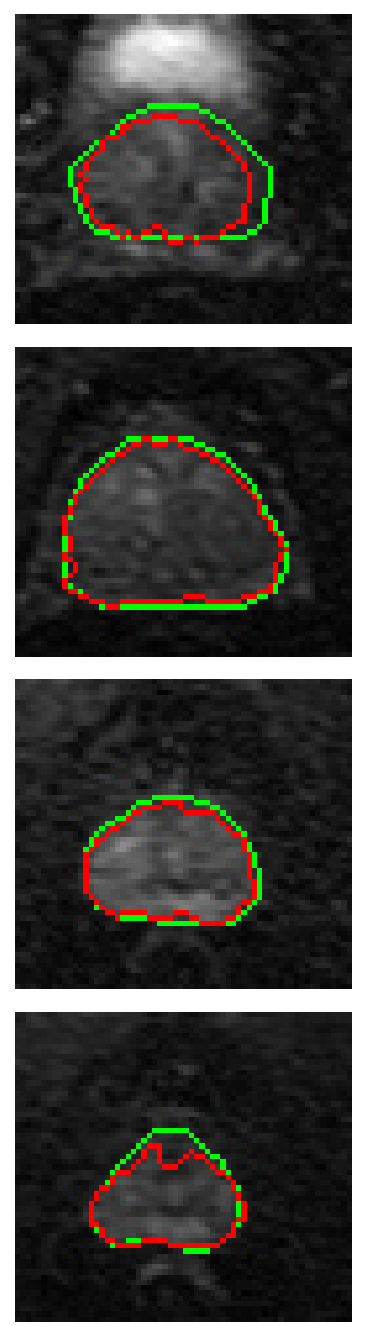

Figure 10. Sample segmentation results presented in $2 \mathrm{D}$ for visualization of the 3D segmentation performed by the proposed nonnegative matrix factorization (NMF)-based level-set approach at different cross sections for 5 different subjects where the green and red curves correspond to the ground truth and our segmentation, respectively.

\section{Segmentation Results}

Evaluation of the system was done using a leave-one-out methodology, where 8 subjects were used as training data and the remaining subject was used as test data. This was repeated so that each subject was tested once. Sample 2D cross sections of the 3D segmentations generated using the proposed approach for different 

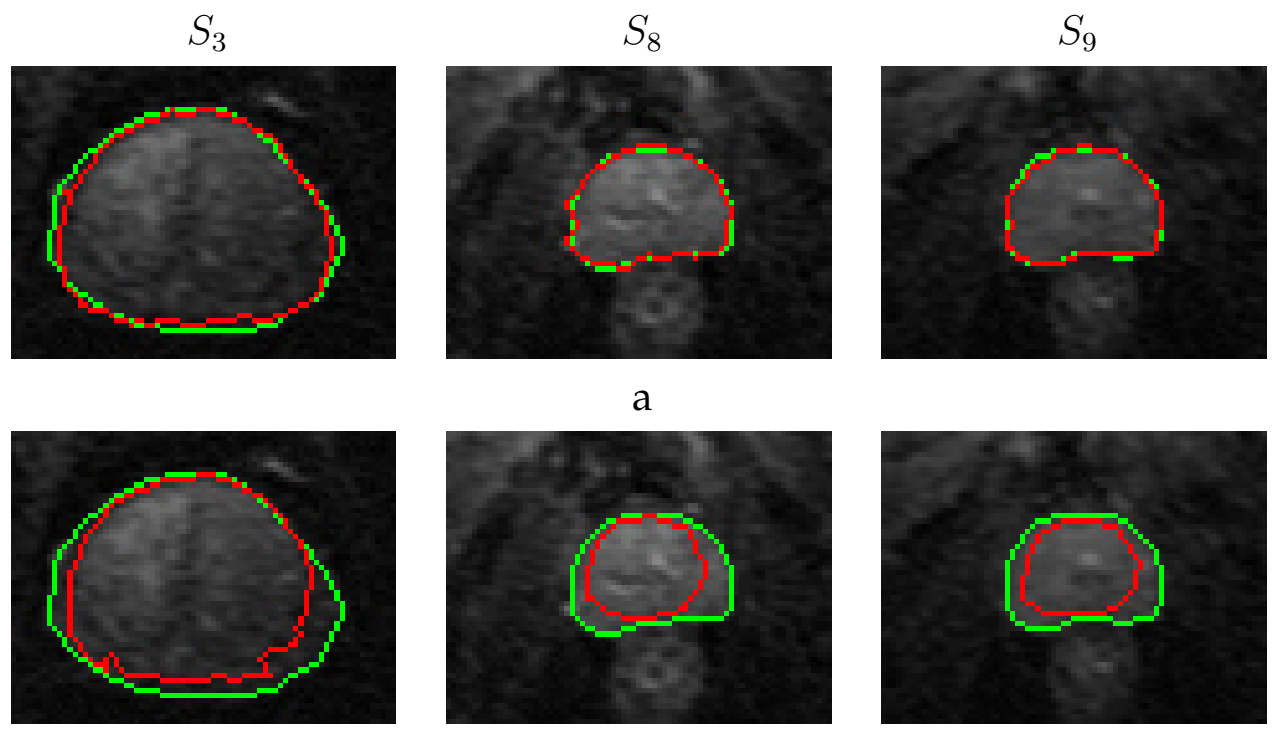

b

Figure 11. Example 2D projections of the 3D segmentation for 3 different patients using the (a) NMF and (b) MAP guided level-set where the green and red curves correspond to the ground truth and segmentation, respectively.

subjects are shown in Fig. 10. In order to evaluate the proposed method, its performance has been compared to two different DWI prostate segmentation methods: (1) the reported results for the 3D approach developed by Liu et al. [179] and (2) a level-set guided by the MAP model proposed by [67] that utilized the probability that a voxel was object or background based on its intensity, shape, and spatial information. Note that the MAP-based method was tested on the same data as the NMF-based approach, but the technique proposed by Liu et al. [179] was tested on a different data set. The average DSC and HD values of the three compared methods are shown in Table 8. Additionally, an example is given in Fig. 11 that contrasts the segmentations of the NMF-based and MAP-based approaches. The evaluation metrics for these two approaches corresponding to each subject are shown in Table 6 and Table 7. Also, the final 3D segmentations of two of the prostates in the data set are shown in Fig. 12.

In addition to DSC and HD, another common metric for evaluating seg- 
TABLE 6. The DSC segmentation performances of the NMF and MAP guided level-set methods for each of the subjects, $S_{i}$ where $i=1 \ldots 9$.

\begin{tabular}{|c|c|c|c|c|c|c|c|c|c|}
\hline Method & $S_{1}$ & $S_{2}$ & $S_{3}$ & $S_{4}$ & $S_{5}$ & $S_{6}$ & $S_{7}$ & $S_{8}$ & $S_{9}$ \\
\hline NMF & 0.822 & 0.861 & 0.907 & 0.862 & 0.905 & 0.828 & 0.851 & 0.886 & 0.905 \\
\hline MAP & 0.816 & 0.858 & 0.881 & 0.836 & 0.900 & 0.827 & 0.849 & 0.647 & 0.880 \\
\hline
\end{tabular}
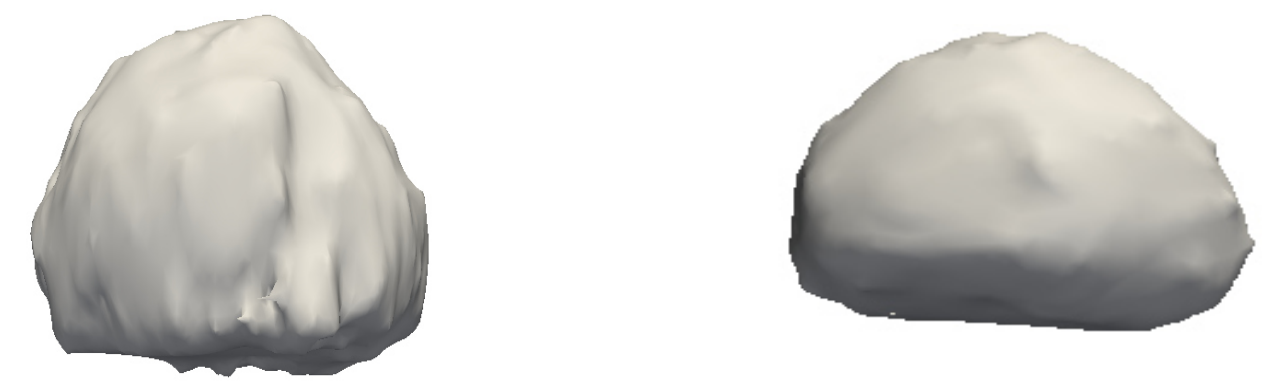

Figure 12. Two example 3D prostate segmentation visualizations.

TABLE 7. The HD segmentation performances of the NMF and MAP guided levelset methods for each of the subjects, $S_{i}$ where $i=1 \ldots 9$.

\begin{tabular}{|c|c|c|c|c|c|c|c|c|c|}
\hline Method & $S_{1}$ & $S_{2}$ & $S_{3}$ & $S_{4}$ & $S_{5}$ & $S_{6}$ & $S_{7}$ & $S_{8}$ & $S_{9}$ \\
\hline NMF & 5.30 & 3.00 & 6.00 & 6.00 & 5.96 & 8.72 & 9.75 & 3.49 & 3.25 \\
\hline MAP & 5.30 & 3.00 & 6.34 & 6.00 & 6.93 & 8.75 & 9.08 & 9.27 & 6.00 \\
\hline
\end{tabular}

TABLE 8. A comparison of the average DSC and HD values over all subjects for the compared methods.

\begin{tabular}{|c|c|c|c|}
\hline Metric & NMF & MAP & Liu et al. [179] \\
\hline DSC & $0.870 \pm 0.03$ & $0.833 \pm 0.07$ & $0.810 \pm 0.05$ \\
\hline $\mathrm{HD}\left(\mathrm{mm}^{3}\right)$ & $5.72 \pm 2.35$ & $6.74 \pm 2.04$ & $9.07 \pm 1.64$ \\
\hline
\end{tabular}




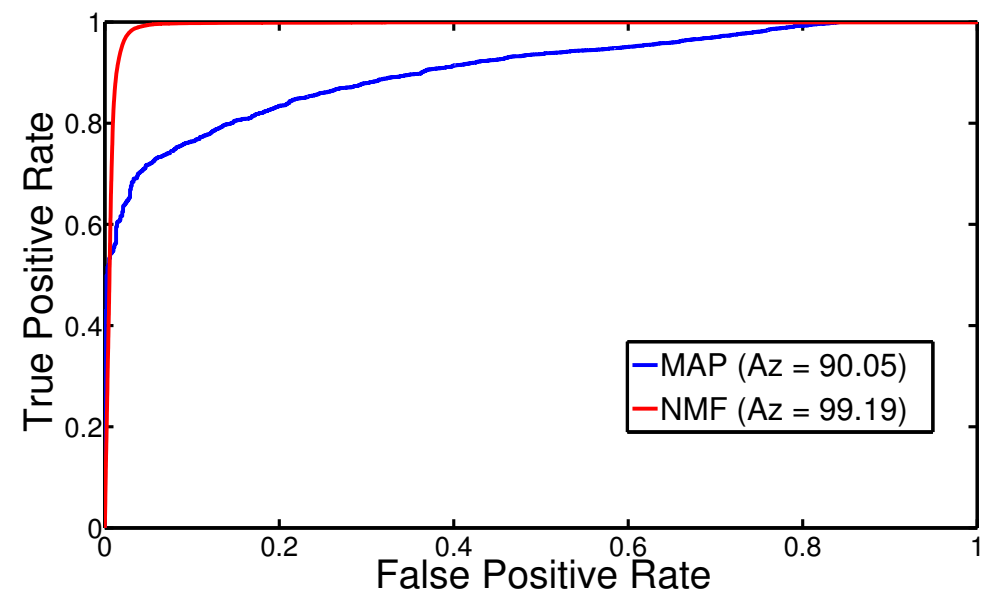

Figure 13. Sample ROC curve for the proposed (red) and the MAP-based (blue) level-set segmentation approaches.

mentations is the receiver operating characteristic (ROC). The ROC measures the sensitivity of a segmentation using different classification thresholds by demonstrating the interaction between the ration of the TP and FP rates. The ROC curves of both the NMF and MAP guiding forces for subject 3, as well as the areas under the curves (Az), are shown in Fig. 13. Additionally, an example is given in Fig. 11 that contrasts the segmentations of the NMF-based and MAP-based level-set methods. The evaluation metrics for these two approaches corresponding to each subject are shown in Table 6 and Table 7. Also, the final 3D segmentations of two of the prostates in the data set are shown in Fig. 12.

\section{E Conclusion}

In summary, using 3D intensity, shape, and spatial features combined with NMF-based feature fusion is significantly better at guiding a level-set for DWI prostate segmentation than either using MAP with the same input information or intensity and shape information alone. The addition of NMF-based feature fusion allows the proposed method to perform robust prostate segmentation despite image noise, inter-patient anatomical differences, and the similar intensities of the 
prostate and surrounding tissues. In future work, this segmentation framework will be tested with a larger data set in order to verify its robustness. Also, segmentation will be performed using several different values of the NMF parameter $r$. In addition, the effectiveness of using the proposed method to segment the prostate at varying b-values will be investigated. 


\section{CHAPTER III}

\section{A NOVEL NMF-BASED DWI PROSTATE CANCER DETECTION FRAMEWORK}

In this chapter, a novel framework for detecting cancer in a segmented diffusion weighted imaging (DWI) prostate is proposed. This method uses a large feature space that includes the maximum and mean apparent diffusion coefficient (ADC) and intensity values at b-values of 100, 200, 300, 400, 500, 600 and 700 as well as the mean intensity at a b-value of 0 . Nonnegative matrix factorization (NMF)-based feature fusion is performed to determine the most discriminant features and cluster the data in a lower-dimensional space. After this, a probabilistic classifier based on the k-nearest neighbors $(\mathrm{kNN})$ and k-means methods is used to label subjects as malignant (i.e. containing cancer) or benign (i.e. containing no cancer). Experimentation shows that the use of NMF-based feature fusion improved the separability of the feature space and results in increased classification accuracy. A traditional kNN classifier achieved an accuracy of 0.667 , while the NMF-based classifier achieved an accuracy of $0.833 \pm 0.078$.

\section{A Introduction}

Large amounts of information can be retrieved from DWI data sets (e.g. mean, maximum, and minimum image intensities and ADC values) for each bvalue pair, as well as the intensity information at the reference b-value, usually 0 . In particular, the ADC is a common feature in prostate cancer CAD systems $[67,69-$ $73,225,226]$. (The equation to calculate the ADC can be found in Chapter 1.) The 
$\mathrm{ADC}$ is the measure of the diffusion of water through tissues and is calculated by comparing DWI magnetic resonance (MR) images taken using magnetic fields with different field strengths (i.e. b-values). Several techniques can be used to extract the most meaningful features or weight the features in accordance with their discriminatory power. Common techniques are k-means clustering [167], principle component analysis (PCA) [167], and information gain [268]. Another technique that has been proposed for this task is NMF. In this chapter, an NMF-based classification framework is proposed for detecting cancer in prostates that have been segmented from DWI volumes.

\section{B Methods}

In this section, the proposed NMF-based DWI prostate cancer detection framework (Fig. 14) is described in detail. This approach has three main steps: (1) NMF-based feature fusion, (2) classification, and (3) refinement. The input to this system is a set of DWI volumes with the prostate segmented. ADC maps, discussed in Chapter 1, were calculated for each segmented prostate using a b-value of 0 as reference.

\section{NMF}

As in the previous chapter, NMF was used to learn a transformation from the original feature space to a lower dimensional space where the data classes are better separated. As before, the multiplicative gradient descent algorithm [265] was used to approximate a weight matrix $W$ for an input matrix $A$ such that $A \approx W H$. The columns of the input matrix $A$ corresponded to the mean and maximum image intensities and ADC values at b-values of 100, 200, 300, 400, 500, 600 , and 700 as well as the mean intensity at a b-value of 0 . In the proposed approach, the feature vectors of both training and testing data are included in $A$. As in the segmentation approach proposed in the previous chapter, $r$, the dimension- 




Figure 14. Diagram of the DWI NMF-based cancer detection framework for prostate cancer.

ality of the transformation space, was set to 3. Classification of a new subject was performed using the k-nearest neighbors (kNN) [167] algorithm. This method was used instead of a $W^{T}$-based approach, similar to the technique described in the previous chapter, because there was better 3D data separation in $H$-space versus $H_{A}$-space where $H_{A}=W^{T} A$. This is illustrated in Fig. 15. Once NMF was performed, the resulting $H$ matrix was used as the input to the classification step.

\section{Classification}

Once each data sample was transformed to $H$-space, classification of benign and malignant subjects was performed using a probabilistic model derived using the kNN algorithm and the distances to class centroids. Each subject $S_{i}$ was given a label $L$ as benign (0) or malignant (1) per the following rule: 


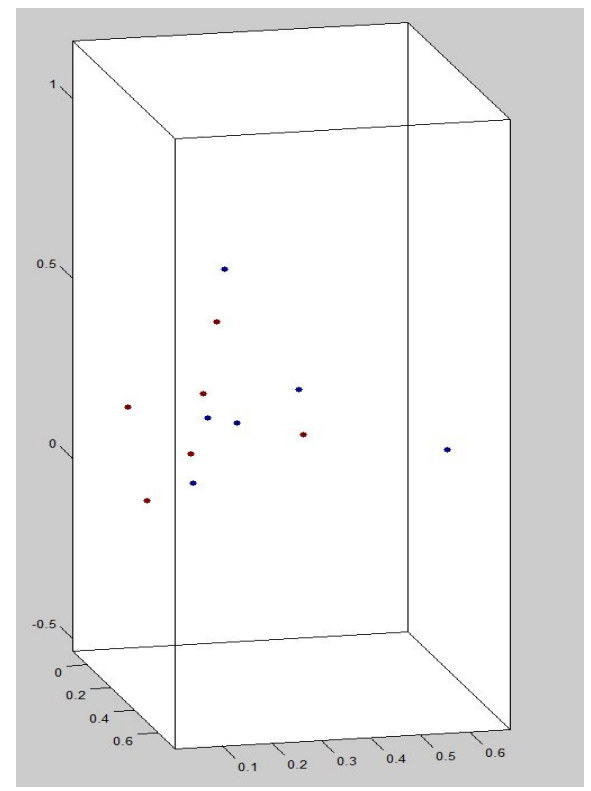

(a)

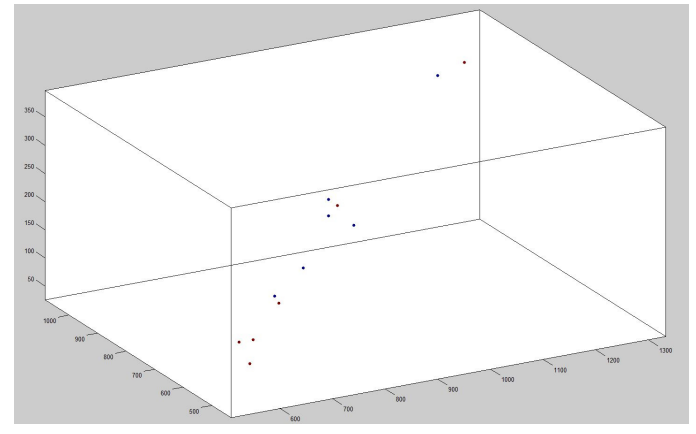

(b)

Figure 15. Example H-space vectors for the 12 subjects created using $W$ (a) and $W^{T}$ (b) where the blue points correspond to benign subjects and the red points correspond to subjects with a malignant tumor. 


$$
L\left(S_{i}\right)= \begin{cases}1 & \text { if } P_{1: S_{i}}>P_{0: S_{i}} \\ 0 & \text { otherwise }\end{cases}
$$

Here, $P_{1: S_{i}}=P_{k n n}\left(1: S_{i}\right) * P_{c}\left(1: S_{i}\right)$ and $P_{0: S_{i}}=P_{k n n}\left(0: S_{i}\right) * P_{c}\left(0: S_{i}\right)$ where $P_{k n n}\left(1: S_{i}\right)$ and $P_{k n n}\left(0: S_{i}\right)$ are the kNN-based probabilities that the subject is malignant or benign, respectively, and $P_{c}\left(1: S_{i}\right)$ and $P_{c}\left(0: S_{i}\right)$ are the centroidbased probabilities that the subject is malignant or benign, respectively. The kNNbased probabilities were calculated by finding the $k=5$ training subjects with the smallest Euclidean distance to a test subject $S_{i}$ in $H$-space. The number of $k$ nearest training points with label $l$ is defined as $n_{l}$ and is used to estimate the label probabilities as defined by:

$$
\begin{aligned}
P_{k n n}\left(1 \mid S_{i}\right) & =\frac{n_{l}}{k} \\
P_{k n n}\left(0 \mid S_{i}\right) & =\frac{n_{0}}{k}
\end{aligned}
$$

The centroid-based probabilities were calculated by finding the Euclidean distances of a test subject $S_{i}$ to the centroids of the malignant and benign training subjects in $H$-space, $d_{1}$ and $d_{0}$, respectively. The corresponding label probabilities are defined as follows:

$$
\begin{aligned}
& P_{c}\left(1 \mid S_{i}\right)=\frac{\frac{1}{d_{1}}}{\frac{1}{d_{1}}+\frac{1}{d_{0}}} \\
& P_{c}\left(0 \mid S_{i}\right)=\frac{\frac{1}{d_{0}}}{\frac{1}{d_{1}}+\frac{1}{d_{0}}}
\end{aligned}
$$

\section{Refinement}

Due to the random initialization of $W$ and $H$, the use of gradient descent, and the low number of data points, the accuracy of classifying in $H$-space significantly varied when NMF was performed. In order to overcome these issues and classify more consistently, Algorithm 3 was used to determine the final classification of a subject. 
Algorithm 3 Algorithm for Refining NMF-Based Classification

Determine the label $L$ of $S_{i}$ by:

1. Calculate $W$ and $H$ using NMF on the training and testing data.

2. Calculate the k-means-based and the kNN-based probabilities of $S_{i}$.

3. Label $S_{i}$ according to the class probabilities (Eq. 17).

4. Repeat Steps 1-3 $\tau$ times

5. Combine the $\tau$ results using an ensemble-based method [269] to classify $S_{i}$

\section{Results}

Testing was performed using a leave-one-out methodology and 12 subjects, each with a DWI scan at b-values ranging from 0 to 700 . 6 of the subjects were malignant and 6 were benign. The above approach was tested with a refinement using $\tau=10$. For comparison, kNN classification without NMF-based feature fusion was performed using only the input data, similar to the approach proposed by Firjani et al. [67]. The accuracies of these methods are shown in Table 9. It may be noted that the minimum accuracy of this approach is equivalent to the accuracy of the kNN method. In addition, the mode accuracy of the NMF approach was 0.917 , occurring in 4 of the 10 runs.

TABLE 9. A comparison of the accuracies of the kNN classifier without NMF and the NMF-based method with refinement (NMF+R).

\begin{tabular}{|c|c|c|}
\hline Method & ACC & Range \\
\hline kNN & 0.667 & - \\
\hline NMF+R & $0.833 \pm 0.078$ & $0.667-0.917$ \\
\hline
\end{tabular}




\section{Conclusion}

In this chapter, a novel NMF-based DWI prostate cancer detection framework was proposed. It was shown to improve upon the use of a traditional $\mathrm{kNN}$ classifier in high-dimensional space. This was achieved by reducing the dimensionality of the classification space by using NMF-based feature fusion. In addition, it is shown that the use of NMF leads to better clustering of malignant and benign data points allowing for increased classification accuracy. Also, this approach shows that the fusion of DWI features from multiple b-values can be used for detecting prostate cancer. To further test and validate this approach, it should be tested on larger data sets. Future work will also include testing using high bvalues $(\geq 800)$ and investigating techniques to improve the consistency of the NMF approximation of $W$, such by using alternative initialization procedures. 


\section{CHAPTER IV}

\section{CONCLUSION AND FUTURE WORK}

In this thesis, a novel computer aided diagnostic (CAD) framework was proposed for detecting prostate cancer in diffusion weighted imaging (DWI) data. This method had two main components: (1) a framework for DWI prostate segmentation and (2) a framework for cancer detection. One major contribution of this work is the use of nonnegative matrix factorization (NMF) to find the most discriminating attributes in high-dimensional feature spaces and combine them in order to create a lower-dimensional space where classes were better clustered and training data was encoded. Specifically:

- In the segmentation component, NMF-based feature fusion of three dimensional (3D) intensity, shape, and spatial information was utilized to guide the evolution of a 3D level-set using a novel probabilistic speed function. The proposed 3D appearance-based shape model takes into consideration both the ground truth segmentation as well as the intensity similarity of voxels when constructing a shape prior. Additionally, the spatial information was modeled using a $2^{\text {nd }}$ order Markov-Gibbs random field (MGRF).

- In the cancer detection component, NMF-based feature fusion was used to extract meaningful features from a large attribute space consisting of intensity and ADC information at a wide range of $b$-values. Also, a probabilistic classifier that takes advantage of the clustering of classes performed by NMF was proposed. 
For both frameworks, experimentation found that the NMF-based approaches were more accurate than other traditional methods. Another contribution of this work is the investigation of combining the information of several different $b$-values in classification. The classification results show that this is promising for discriminating between malignant and benign prostates using DWI data.

In addition, several possibilities for future work relating to and extending this thesis are:

- Testing the proposed segmentation and classification frameworks on larger data sets, with a wider range of b-values, and using a variety of method parameters.

- Integrating the proposed NMF-based frameworks into a contiguous CAD system for prostate cancer and testing this system.

- Applying these NMF-based frameworks to other medical image analysis applications such as dyslexia [87, 109, 120,270-275], autism [111,123, 276-285], acute renal rejection $[88,183,184,202,286-302]$, and lung cancer [114, 141, 142, 262, 303, 304, ?, 304, ?, 304, ?, 304, ?, 304, ?, 304, ?, 304, ?, 304, ?, 304, ?-306, ?-306, ?306, ?-306, ?-306, ?-306, ?-306, ?-306, ?-307, ?-307, ?-307, ?-307, ?-307]. 


\section{REFERENCES}

[1] Rebecca Siegel, Deepa Naishadham, and Ahmedin Jemal, "Cancer statistics 2013," CA-Cancer J. Clin., vol. 63, no. 1, pp. 11-30, 2013.

[2] Paolo Vineis and Christopher P Wild, "Global cancer patterns: causes and prevention," Lancet, 2013.

[3] Mitchell S. Wachtel, Thomas Nelius, Allan L. Haynes, Scott Dahlbeck, and Werner de Riese, "PSA screening and deaths from prostate cancer after diagnosis: a population based analysis," Prostate, vol. 73, no. 12, pp. 1365-1369, 2013.

[4] R Etzioni, R Gulati, MR Cooperberg, DM Penson, NS Weiss, and IM Thompson, "Limitations of basing screening policies on screening trials: The US preventive services task force and prostate cancer screening," Med. Care, vol. 51, no. 4, pp. 295-300, 2013.

[5] Michael J Barry, "Prostate-specific-antigen testing for early diagnosis of prostate cancer," N. Engl. J. Med., vol. 344, no. 18, pp. 1373-1377, 2001.

[6] David Shier, Jackie Butler, and Ricki Lewis, Hole's essentials of human anatomy and physiology, McGraw-Hill New York, 2006.

[7] Kai H Hammerich, Gustavo E Ayala, and Thomas M Wheeler, "Anatomy of the prostate gland and surgical pathology of prostate cancer," Cambridge University, Cambridge, pp. 1-10, 2009.

[8] John E McNeal, "The zonal anatomy of the prostate," Prostate, vol. 2, no. 1, pp. 35-49, 1981. 
[9] John E McNeal, "Normal histology of the prostate.," The Am. J. Surg. Pathol., vol. 12 , no. 8, pp. 619-633, 1988.

[10] Steven H Selman, "The McNeal prostate: a review," Urology, vol. 78, no. 6, pp. 1224-1228, 2011.

[11] Christine H Lee, Oluyemi Akin-Olugbade, and Alexander Kirschenbaum, "Overview of prostate anatomy, histology, and pathology," Endocrinol. Metab. Clin. North Am., vol. 40, no. 3, pp. 565-575, 2011.

[12] S Dijkstra, PFA Mulders, and JA Schalken, "Clinical use of novel urine and blood based prostate cancer biomarkers: A review," Clin. Biochem., 2013.

[13] Glenn S Gerber, Ian M Thompson, Ronald Thisted, and Gerald W Chodak, "Disease-specific survival following routine prostate cancer screening by digital rectal examination," J. American Med. Assoc., vol. 269, no. 1, pp. 61-64, 1993.

[14] Ulf-Håkan Stenman, Jari Leinonen, Wan-Ming Zhang, and Patrik Finne, "Prostate-specific antigen," in Semin. Cancer Biol. Elsevier, 1999, vol. 9, pp. 83-93.

[15] Arun Sreekumar, Bharathi Laxman, Daniel R Rhodes, Srilakshmi Bhagavathula, Jason Harwood, Donald Giacherio, Debashis Ghosh, Martin G Sanda, Mark A Rubin, and Arul M Chinnaiyan, "Humoral immune response to $\alpha$-methylacyl-CoA racemase and prostate cancer," J. Natl. Cancer Inst., vol. 96, no. 11, pp. 834-843, 2004.

[16] Brian V Le, Christopher R Griffin, Stacy Loeb, Gustavo F Carvalhal, Donghui Kan, Nikola A Baumann, and William J Catalona, "[-2] proenzyme prostate specific antigen is more accurate than total and free prostate specific antigen in differentiating prostate cancer from benign disease in a prospective prostate cancer screening study," J. Urol., vol. 183, no. 4, pp. 1355-1359, 2010. 
[17] William J Catalona, Alan W Partin, Martin G Sanda, John T Wei, George G Klee, Chris H Bangma, Kevin M Slawin, Leonard S Marks, Stacy Loeb, Dennis L Broyles, Sanghyuk S Shin, Amabelle B Cruz, Daniel W Chan, Lori J Sokoll, William L Roberts, Ron HN van Schaik, and Isaac A Mizrahi, "A multicenter study of [-2] pro-prostate specific antigen combined with prostate specific antigen and free prostate specific antigen for prostate cancer detection in the 2.0 to $10.0 \mathrm{ng} / \mathrm{ml}$ prostate specific antigen range," J. of Urol., vol. 185, no. 5, pp. 1650-1655, 2011.

[18] Felix K Chun, Alexandre de la Taille, Hendrik Van Poppel, Michael Marberger, Arnulf Stenzl, Peter FA Mulders, Hartwig Huland, Clement-Claude Abbou, Alexander B Stillebroer, Martijn PMQ van Gils, Jack A. Schalken, Yves Fradet, Leonard S Marks, William Ellis, Alan W. Partin, and Alexander Haese, "Prostate cancer gene 3 ( PCA3): Development and internal validation of a novel biopsy nomogram," Eur. Urol., vol. 56, no. 4, pp. 659-668, 2009.

[19] Ina L Deras, Sheila MJ Aubin, Amy Blase, John R Day, Seongjoon Koo, Alan W Partin, William J Ellis, Leonard S Marks, Yves Fradet, Harry Rittenhouse, and Jack Groskopf, "PCA3: a molecular urine assay for predicting prostate biopsy outcome," J. Urol., vol. 179, no. 4, pp. 1587-1592, 2008.

[20] Martijn PMQ van Gils, Daphne Hessels, Onno van Hooij, Sander A Jannink, W Pim Peelen, Suzanne LJ Hanssen, J Alfred Witjes, Erik B Cornel, Herbert FM Karthaus, Geert AHJ Smits, Gerhard A Dijkman, Peter FA Mulders, and Jack A Schalken, "The time-resolved fluorescence-based PCA3 test on urinary sediments after digital rectal examination; a dutch multicenter validation of the diagnostic performance," Clin. Cancer Res., vol. 13, no. 3, pp. 939-943, 2007.

[21] Monique J. Roobol, Fritz H. Schröder, Pim van Leeuwen, Tineke Wolters, 
Roderick C.N. van den Bergh, Geert JLH van Leenders, and Daphne Hessels, "Performance of the prostate cancer antigen 3 (PCA3) gene and prostatespecific antigen in prescreened men: Exploring the value of PCA3 for a firstline diagnostic test," Eur. Urol., vol. 58, no. 4, pp. 475-481, 2010.

[22] Alexander Haese, Alexandre de la Taille, Hendrik Van Poppel, Michael Marberger, Arnulf Stenzl, Peter FA Mulders, Hartwig Huland, Clément-Claude Abbou, Mesut Remzi, Martina Tinzl, Susan Feyerabend, Alexander B Stillebroer, Martijn PMQ van Gils, and Jack A Schalken, "Clinical utility of the PCA3 urine assay in european men scheduled for repeat biopsy," Eur. Urol., vol. 54, no. 5, pp. 1081-1088, 2008.

[23] Donna Pauler Ankerst, Jack Groskopf, John R Day, Amy Blase, Harry Rittenhouse, Brad H Pollock, Cathy Tangen, Dipen Parekh, Robin J Leach, and Ian Thompson, "Predicting prostate cancer risk through incorporation of prostate cancer gene 3," J. Urol., vol. 180, no. 4, pp. 1303-1308, 2008.

[24] Simpa S Salami, Folke Schmidt, Bharathi Laxman, Meredith M Regan, David S Rickman, Douglas Scherr, Gerardina Bueti, Javed Siddiqui, Scott A Tomlins, John T Wei, Arul M Chinnaiyan, and Martin G Rubin, Mark A ande Sandra, "Combining urinary detection of TMPRSS2: ERG and PCA3 with serum PSA to predict diagnosis of prostate cancer," in Uro. Oncol. Semin. Ori. Elsevier, 2011.

[25] Leonard S Marks, Yves Fradet, Ina Lim Deras, Amy Blase, Jeannette Mathis, Sheila MJ Aubin, Anthony T Cancio, Marie Desaulniers, William J Ellis, Harry Rittenhouse, and Jack Groskopf, "PCA3 molecular urine assay for prostate cancer in men undergoing repeat biopsy," Urology, vol. 69, no. 3, pp. 532-535, 2007.

[26] David Ulmert, Angel M Cronin, Thomas Björk, Matthew F O’Brien, Peter T 
Scardino, James A Eastham, Charlotte Becker, Göran Berglund, Andrew J Vickers, and Hans Lilja, "Prostate-specific antigen at or before age 50 as a predictor of advanced prostate cancer diagnosed up to 25 years later: a casecontrol study," BMC Med., vol. 6, no. 1, pp. 6, 2008.

[27] Jonas Hugosson, Sigrid Carlsson, Gunnar Aus, Svante Bergdahl, Ali Khatami, Pär Lodding, Carl-Gustaf Pihl, Johan Stranne, Erik Holmberg, and Hans Lilja, "Mortality results from the göteborg randomised populationbased prostate-cancer screening trial," Lancet Oncol., vol. 11, no. 8, pp. 725$732,2010$.

[28] Matteo Ferro, Dario Bruzzese, Sisto Perdona, Ada Marino, Claudia Mazzarella, Giuseppe Perruolo, Vittoria DEsposito, Vincenzo Cosimato, Carlo Buonerba, Giuseppe Di Lorenzo, Gennaro Musi, Ottavio De Cobelli, Felix K Chun, and Daniela Terracciano, "Prostate health index (PHI) and prostate cancer antigen 3 (PCA3) significantly improve prostate cancer detection at initial biopsy in a total PSA range of 2-10 ng/ml," PloS One, vol. 8, no. 7, pp. e67687, 2013.

[29] Cuvillier Olivier and Malavaud Bernard, "Biomarkers of aggressiveness in prostate cancer," Prostate Cancer-Diagn. Ther. Adv., p. 3, 2011.

[30] Girish Sardana, Barry Dowell, and Eleftherios P Diamandis, "Emerging biomarkers for the diagnosis and prognosis of prostate cancer," Clin. Chem., vol. 54, no. 12, pp. 1951-1960, 2008.

[31] Hirotsugu Uetsuki, Hiroyuki Tsunemori, Rikiya Taoka, Reiji Haba, Masashi Ishikawa, and Yoshiyuki Kakehi, "Expression of a novel biomarker, EPCA, in adenocarcinomas and precancerous lesions in the prostate," J. Urol., vol. 174, no. 2, pp. 514-518, 2005. 
[32] Dipen J Parekh, Donna Pauler Ankerst, Dean Troyer, Sudhir Srivastava, and Ian M Thompson, "Biomarkers for prostate cancer detection," J. Urol., vol. 178, no. 6, pp. 2252-2259, 2007.

[33] Barbara Paul, Rajiv Dhir, Douglas Landsittel, Moira R Hitchens, and Robert H Getzenberg, "Detection of prostate cancer with a blood-based assay for early prostate cancer antigen," Cancer Res., vol. 65, no. 10, pp. 40974100, 2005.

[34] Mark A Rubin, Tarek A Bismar, Ove Andrén, Lorelei Mucci, Robert Kim, Ronglai Shen, Debashis Ghosh, John T Wei, Arul M Chinnaiyan, Hans-Olov Adami, Philip W. Kantoff, and Jan-Erik Johansson, “Decreased $\alpha$-methylacyl CoA racemase expression in localized prostate cancer is associated with an increased rate of biochemical recurrence and cancer-specific death," Cancer Epidemiol., Biomarkers Prev., vol. 14, no. 6, pp. 1424-1432, 2005.

[35] Po-Yuan Lin, Kai-Lun Cheng, James D McGuffin-Cawley, Fuh-Sheng Shieu, Anna C Samia, Sanjay Gupta, Matthew Cooney, Cheryl L Thompson, and Chung Chiun Liu, "Detection of alpha-methylacyl-CoA racemase (AMACR), a biomarker of prostate cancer, in patient blood samples using a nanoparticle electrochemical biosensor," Biosensors, vol. 2, no. 4, pp. 377$387,2012$.

[36] Marco Auprich, Anders Bjartell, Felix K-H Chun, Alexandre de la Taille, Stephen J Freedland, Alexander Haese, Jack Schalken, Arnulf Stenzl, Bertrand Tombal, and Henk van der Poel, "Contemporary role of prostate cancer antigen 3 in the management of prostate cancer," Eur. Urol., vol. 60, no. 5, pp. 1045-1054, 2011.

[37] Daphne Hessels, Jacqueline MT Klein Gunnewiek, Inge van Oort, Herbert FM Karthaus, Geert JL van Leenders, Bianca van Balken, Lambertus A 
Kiemeney, J Alfred Witjes, and Jack A Schalken, “Dd3 (pca3)-based molecular urine analysis for the diagnosis of prostate cancer," Eur. Urol., vol. 44, no. 1, pp. 8-16, 2003.

[38] E David Crawford, Kyle O Rove, Edouard J Trabulsi, Junqi Qian, Krystyna P Drewnowska, Jed C Kaminetsky, Thomas K Huisman, Mark L Bilowus, Sheldon J Freedman, W Lloyd Glover Jr, and David G. Bostwick, "Diagnostic performance of PCA3 to detect prostate cancer in men with increased prostate specific antigen: a prospective study of 1,962 cases," J. Urol., vol. 188, no. 5, pp. 1726-1731, 2012.

[39] Jack Groskopf, Sheila MJ Aubin, Ina Lim Deras, Amy Blase, Sharon Bodrug, Craig Clark, Steven Brentano, Jeannette Mathis, Jimmykim Pham, Troels Meyer, Michelle Cass, Petrea Hodge, Maria Luz Macairan, Leonard S Marks, and Harry Rittenhouse, "APTIMA PCA3 molecular urine test: development of a method to aid in the diagnosis of prostate cancer," Clin. Chem., vol. 52, no. 6, pp. 1089-1095, 2006.

[40] Gisele HJM Leyten, Daphne Hessels, Sander A Jannink, Frank P Smit, Hans de Jong, Erik B Cornel, Theo M de Reijke, Henk Vergunst, Paul Kil, Ben C Knipscheer, Inge M. van Oort, Peter FA Mulders, Christina A Hulsbergenvan de Kaa, and Jack A Schalken, "Prospective multicentre evaluation of PCA3 and TMPRSS2-ERG gene fusions as diagnostic and prognostic urinary biomarkers for prostate cancer," Eur. Urol., 2012.

[41] Carsten Stephan, Klaus Jung, Axel Semjonow, Kai Schulze-Forster, Henning Cammann, Xinhai Hu, Hellmuth-A Meyer, Martin Bögemann, Kurt Miller, and Frank Friedersdorff, "Comparative assessment of urinary prostate cancer antigen 3 and TMPRSS2: ERG gene fusion with the serum [-2] proprostate-specific antigen-based prostate health index for detection of prostate cancer," Clinical Chemistry, vol. 59, no. 1, pp. 280-288, 2013. 
[42] Scott A Tomlins, Daniel R Rhodes, Sven Perner, Saravana M Dhanasekaran, Rohit Mehra, Xiao-Wei Sun, Sooryanarayana Varambally, Xuhong Cao, Joelle Tchinda, Rainer Kuefer, Charles Lee, James E Montie, Rajal B. Shah, Kenneth J. Pienta, Mark A. Rubin, and Arul M Chinnaiyan, "Recurrent fusion of TMPRSS2 and ETS transcription factor genes in prostate cancer," Science, vol. 310, no. 5748, pp. 644-648, 2005.

[43] Daniel W Lin, Lisa F Newcomb, Elissa C Brown, James D Brooks, Peter R Carroll, Ziding Feng, Martin E Gleave, Raymond S Lance, Martin G Sanda, Ian M Thompson, John T Wei, and Peter S Nelson, “Urinary TMPRSS2: ERG and PCA3 in an active surveillance cohort: results from a baseline analysis in the canary prostate active surveillance study," Clin. Cancer Res., vol. 19, no. 9, pp. 2442-2450, 2013.

[44] David W Hosmer Jr and Stanley Lemeshow, Applied logistic regression, John Wiley \& Sons, 2004.

[45] Marco Auprich, Alexander Haese, Jochen Walz, Karl Pummer, Alexandre de la Taille, Markus Graefen, Theo de Reijke, Margit Fisch, Paul Kil, Paolo Gontero, Jacques Irani, and Chun Felix KH, “External validation of urinary PCA3-based nomograms to individually predict prostate biopsy outcome," Eur. Urol., vol. 58, no. 5, pp. 727-732, 2010.

[46] Sisto Perdonà, Vitor Cavadas, Giuseppe Di Lorenzo, Rocco Damiano, Gennaro Chiappetta, Paola Del Prete, Renato Franco, Giuseppina Azzarito, Stefania Scala, Claudio Arra, Marco De Sio, and Riccardo Autorino, "Prostate cancer detection in the grey area of prostate-specific antigen below $10 \mathrm{ng} / \mathrm{ml}$ : head-to-head comparison of the updated PCPT calculator and Chun's nomogram, two risk estimators incorporating prostate cancer antigen 3," Eur. Urol., vol. 59, no. 1, pp. 81-87, 2011. 
[47] Jens Hansen, Marco Auprich, Sascha A Ahyai, Alexandre de la Taille, Hendrik Van Poppel, Michael Marberger, Arnulf Stenzl, Peter FA Mulders, Hartwig Huland, Margit Fisch, Clement-Claude Abbou, Jack A Schalken, Yves Fradet, Leonard S Marks, William Ellis, Alan W Partin, Karl Pummer, Markus Graefen, Alexander Haese, Jochen Walz, Alberto Briganti, Shahrokh F Shariat, and Felix K Chun, "Initial prostate biopsy: development and internal validation of a biopsy-specific nomogram based on the prostate cancer antigen 3 assay," Eur. Urol., vol. 63, no. 2, pp. 201-209, 2013.

[48] Hiroyuki Nakanishi, Jack Groskopf, Herbert A Fritsche, Viju Bhadkamkar, Amy Blase, S. Vikas Kumar, John W Davis, Patricia Troncoso, Harry Rittenhouse, and R. Joseph Babaian, "PCA3 molecular urine assay correlates with prostate cancer tumor volume: implication in selecting candidates for active surveillance," J. Urol., vol. 179, no. 5, pp. 1804-1810, 2008.

[49] Marco Auprich, Felix K.H. Chun, John F Ward, Karl Pummer, Richard Babaian, Herbert Augustin, Ferdinand Luger, Stefan Gutschi, Lars Budäus, Margit Fisch, Hartwig Huland, Markus Graefen, and Alexander Haese, "Critical assessment of preoperative urinary prostate cancer antigen 3 on the accuracy of prostate cancer staging," Eur. Urol., vol. 59, no. 1, pp. 96-105, 2011.

[50] Jonathan I Epstein, Patrick C Walsh, and Fred Sanfilippo, "Clinical and cost impact of second-opinion pathology: review of prostate biopsies prior to radical prostatectomy," Am. J. Surg. Pathol., vol. 20, no. 7, pp. 851-857, 1996.

[51] Theresa Y Chan and Jonathan I Epstein, "Patient and urologist driven second opinion of prostate needle biopsies," J. Urol., vol. 174, no. 4, pp. 1390-1394, 2005.

[52] Michael Davis, Mario Sofer, Sandy S Kim, and Mark S Soloway, "The procedure of transrectal ultrasound guided biopsy of the prostate: a survey of 
patient preparation and biopsy technique," J. Urol., vol. 167, no. 2, pp. 566$570,2002$.

[53] Angelo M DeMarzo, William G Nelson, William B Isaacs, and Jonathan I Epstein, "Pathological and molecular aspects of prostate cancer," Lancet, vol. 361, no. 9361, pp. 955-964, 2003.

[54] Donald F Gleason and George T Mellinger, "Prediction of prognosis for prostatic adenocarcinoma by combined histological grading and clinical staging," J. Urol., vol. 111, pp. 58-64, 1974.

[55] William C Allsbrook Jr, Kathy A Mangold, Maribeth H Johnson, Roger B Lane, Cynthia G Lane, and Jonathan I Epstein, "Interobserver reproducibility of gleason grading of prostatic carcinoma: general pathologist," Hum. Pathol., vol. 32, no. 1, pp. 81-88, 2001.

[56] Z Jiang, CL Wu, BA Woda, KA Iczkowski, PG Chu, MS Tretiakova, RH Young, LM Weiss, RD Blute, CB Brendler, T Krausz, JC Xu, MB Amin, and XJ Yang, "Alpha-methylacyl-CoA racemase: a multi-institutional study of a new prostate cancer marker," Histopathology, vol. 45, no. 3, pp. 218-225, 2004.

[57] Mikael Kubista, José Manuel Andrade, Martin Bengtsson, Amin Forootan, Jiri Jonák, Kristina Lind, Radek Sindelka, Robert Sjöback, Björn Sjögreen, Linda Strömbom, Anders Ståhlberg, and Neven Zoric, "The real-time polymerase chain reaction," Mol. Asp. Med., vol. 27, no. 2, pp. 95-125, 2006.

[58] Hedvig Hricak, Peter L Choyke, Steven C Eberhardt, Steven A Leibel, and Peter T Scardino, "Imaging prostate cancer: A multidisciplinary perspective," Radiology, vol. 243, no. 1, pp. 28-53, 2007.

[59] Gabor Fichtinger, Axel Krieger, Robert C Susil, Attila Tanacs, Louis L Whitcomb, and Ergin Atalar, "Transrectal prostate biopsy inside closed MRI scan- 
ner with remote actuation, under real-time image guidance," in Proceedings of the International Conference on Medical Image Computing and ComputerAssisted Intervention (MICCAI'02), Tokyo, Japan, September 25-28 2002, pp. 91-98, Springer.

[60] Jeffrey C Applewhite, BR Matlaga, DL McCullough, MC Hall, et al., “Transrectal ultrasound and biopsy in the early diagnosis of prostate cancer.," Cancer Control, vol. 8, no. 2, pp. 141, 2001.

[61] M Fuchsjäger, A Shukla-Dave, O Akin, J Barentsz, and H Hricak, "Prostate cancer imaging," Acta Radiol., vol. 49, no. 1, pp. 107-120, 2008.

[62] Stewart C Bushong, Magnetic resonance imaging, Elsevier Health Sciences, 2003.

[63] Michael Seitz, Amita Shukla-Dave, Anders Bjartell, Karim Touijer, Alessandro Sciarra, Patrick J Bastian, Christian Stief, Hedvig Hricak, and Anno Graser, "Functional magnetic resonance imaging in prostate cancer," Eur. Urol., vol. 55, no. 4, pp. 801-814, 2009.

[64] S Punwani, M Emberton, M Walkden, A Sohaib, A Freeman, H Ahmed, C Allen, and A Kirkham, "Prostatic cancer surveillance following wholegland high-intensity focused ultrasound: comparison of MRI and prostatespecific antigen for detection of residual or recurrent disease," Br. J. Radiol., vol. 85, no. 1014, pp. 720-728, 2012.

[65] Hiroo Kajihara, Yoshiko Hayashida, Ryuji Murakami, Kazuhiro Katahira, Ryuichi Nishimura, Yasuyuki Hamada, Kousuke Kitani, Mitsuhiko Kitaoka, Yasuko Suzuki, Mika Kitajima, et al., "Usefulness of diffusion-weighted imaging in the localization of prostate cancer," Int. J. Radiat. Oncol. Biol. Phys., vol. 74, no. 2, pp. 399-403, 2009. 
[66] Kyle K Yu and Hedvig Hricak, "Imaging prostate cancer," Radiol. Clin. North Am., vol. 38, no. 1, pp. 59-85, 2000.

[67] A. Firjani, A. Elnakib, F. Khalifa, G. Gimmel'farb, M. Abou El-Ghar, A. Elmaghraby, and A. El-Baz, "A diffusion-weighted imaging based diagnostic system for early detection of prostate cancer," J. Biomed. Sci. Eng., vol. 6, no. 3, pp. 346-356, 32013.

[68] Satish Viswanath, B Nicolas Bloch, Mark Rosen, Jonathan Chappelow, Robert Toth, Neil Rofsky, Robert Lenkinski, Elizabeth Genega, Arjun Kalyanpur, and Anant Madabhushi, "Integrating structural and functional imaging for computer assisted detection of prostate cancer on multi-protocol in vivo 3 Tesla MRI," in Proceedings of the SPIE Conference on Medical Imaging 2009, San Diego, California, USA, February 7-12 2009, International Society for Optics and Photonics, p. 72603I.

[69] GJS Litjens, PC Vos, JO Barentsz, N Karssemeijer, and HJ Huisman, “Automatic computer aided detection of abnormalities in multi-parametric prostate MRI," in Proceedings of the SPIE Conference on Medical Imaging 2011. International Society for Optics and Photonics, 2011, pp. 79630T-79630T.

[70] PC Vos, JO Barentsz, N Karssemeijer, and HJ Huisman, "Automatic computer-aided detection of prostate cancer based on multiparametric magnetic resonance image analysis," Phys. Med. Biol., vol. 57, no. 6, pp. 1527, 2012.

[71] Emilie Niaf, Olivier Rouvière, Florence Mège-Lechevallier, Flavie Bratan, and Carole Lartizien, "Computer-aided diagnosis of prostate cancer in the peripheral zone using multiparametric MRI," Phys. Med. Biol., vol. 57, no. 12, pp. 3833, 2012. 
[72] Thomas Hambrock, Pieter C Vos, Christina A Hulsbergen-van de Kaa, Jelle O Barentsz, and Henkjan J Huisman, "Prostate cancer: Computer-aided diagnosis with multiparametric 3-T MR imaging- effect on observer performance," Radiology, vol. 266, no. 2, pp. 521-530, 2013.

[73] Yahui Peng, Yulei Jiang, Cheng Yang, Jeremy Bancroft Brown, Tatjana Antic, Ila Sethi, Christine Schmid-Tannwald, Maryellen L Giger, Scott E Eggener, and Aytekin Oto, "Quantitative analysis of multiparametric prostate MR images: Differentiation between prostate cancer and normal tissue and correlation with Gleason score," Radiology, vol. 267, no. 3, pp. 787-796, 2013.

[74] Vijay Shah, Baris Turkbey, Haresh Mani, Yuxi Pang, Thomas Pohida, Maria J Merino, Peter A Pinto, Peter L Choyke, and Marcelino Bernardo, “Decision support system for localizing prostate cancer based on multiparametric magnetic resonance imaging," Med. Phys., vol. 39, pp. 4093, 2012.

[75] Ahmedin Jemal, Rebecca Siegel, Elizabeth Ward, Yongping Hao, Jiaquan Xu, Taylor Murray, and Michael J Thun, “Cancer statistics, 2008," CA-Cancer J. Clin., vol. 58, no. 2, pp. 71-96, 2008.

[76] Corinna Cortes and Vladimir Vapnik, "Support-vector networks," Mach. Learn., vol. 20, no. 3, pp. 273-297, 1995.

[77] Sheng $X u$, Jochen Kruecker, Baris Turkbey, Neil Glossop, Anurag K Singh, Peter Choyke, Peter Pinto, and Bradford J Wood, "Real-time MRI-TRUS fusion for guidance of targeted prostate biopsies," Comput. Aided Surg., vol. 13, no. 5, pp. 255-264, 2008.

[78] Soumya Ghose, Arnau Oliver, Robert Martí, Xavier Lladó, Joan C Vilanova, Jordi Freixenet, Jhimli Mitra, Désiré Sidibé, and Fabrice Meriaudeau, “A survey of prostate segmentation methodologies in ultrasound, magnetic res- 
onance and computed tomography images," Comput. Methods Progr. Biomed., vol. 108, no. 1, pp. 262-287, 2012.

[79] R Abolmaesumi and MR Sirouspour, "Segmentation of prostate contours from ultrasound images," in Proceedings of the IEEE International Conference on Acoustics, Speech, and Signal Processing (ICASSP'04), Montreal, Quebec, Canada, May 17-21 2004, IEEE, vol. 3, pp. 517-520.

[80] T Kirubarajan, Y Bar-Shalom, WD Blair, and GA Watson, "IMMPDAF for radar management and tracking benchmark with ECM," IEEE Trans. Aerosp. and Electron. Sys., vol. 34, no. 4, pp. 1115-1134, 1998.

[81] Farhang Sahba, Hamid R Tizhoosh, and Magdy MA Salama, "Segmentation of prostate boundaries using regional contrast enhancement," in Proceedings of the IEEE International Conference on Image Processing (ICIP'05), Genoa, Italy, September 11-14 2005, IEEE, vol. 2, pp. 1266-1269.

[82] Rafael C Gonzalez, Richard E Woods, and Steven L Eddins, Digital image processing using MATLAB, vol. 2, Gatesmark Publishing Knoxville, 2009.

[83] Rudolph Emil Kalman, "A new approach to linear filtering and prediction problems," J. Basic Eng., vol. 82, no. 1, pp. 35-45, 1960.

[84] Michael Kass, Andrew Witkin, and Demetri Terzopoulos, "Snakes: Active contour models," Int. J. Comput. Vis., vol. 1, no. 4, pp. 321-331, 1988.

[85] Vicent Caselles, Francine Catté, Tomeu Coll, and Françoise Dibos, "A geometric model for active contours in image processing," Numer. Math., vol. 66, no. 1, pp. 1-31, 1993.

[86] Ayman El-Baz, G Gimel'farb, Robert Falk, and M Abo El-Ghar, "Automatic analysis of 3D low dose CT images for early diagnosis of lung cancer," Pattern Recognit., vol. 42, no. 6, pp. 1041-1051, 2009. 
[87] Ayman El-Baz, Manuel Casanova, G Gimel'farb, Meghan Mott, and A Switala, "An MRI-based diagnostic framework for early diagnosis of dyslexia," Int. J. Computer Assist. Radiol. Surg., vol. 3, no. 3-4, pp. 181-189, 2008.

[88] Seniha E Yuksel, Ayman El-Baz, Aly A Farag, Mohamed El-Ghar, Tarek Eldiasty, and Mohamed A Ghoneim, "A kidney segmentation framework for dynamic contrast enhanced magnetic resonance imaging," J. Vib. Control, vol. 13, no. 9-10, pp. 1505-1516, 2007.

[89] Aly A Farag, Refaat M Mohamed, and Ayman El-Baz, "A unified framework for map estimation in remote sensing image segmentation," IEEE Trans. Geosci. Electron., vol. 43, no. 7, pp. 1617-1634, 2005.

[90] A. El-Baz and G. Gimel'farb, “Image segmentation with a parametric deformable model using shape and appearance priors," in Proceedings of the IEEE Conference on Computer Vision and Pattern Recognition (CVPR'2008), Anchorage, Alaska, USA, June 24-26 2008, IEEE, pp. 1-8.

[91] Ayman El-Baz, Aly A. Farag, and Georgy L. Gimel'farb, "Stochastic deformable model," in Proceedings of the British Machine Vision Conference (BMVC'05), William F. Clocksin, Andrew W. Fitzgibbon, and Philip H. S. Torr, Eds., Oxford, UK, September 5-8 2005, pp. 230-239, British Machine Vision Association.

[92] A. El-Baz, G. Gimel'farb, and A. Abdel-Hakim, “Robust image segmentation with parametric deformable model using learning shape priors," in Proceedings of the ICGST International Conference on Graphics, Vision and Image Processing (GVIP'05), Cairo, Egypt, December 19-21 2005, pp. 558-570.

[93] A. El-Baz, S. Yuksel, H. Shi, A. Farag, M. Abou El-Ghar, T. Eldiasty, and M. Ghoneim, "2D and 3D shape based segmentation using deformable mod- 
els," in Proceedings of the International Conference on Medical Image Computing and Computer-Assisted Intervention (MICCAI'05), Palm Springs, California, USA, October 26-29 2005, vol. 2, pp. 821-829.

[94] Ravi Malladi, James A Sethian, and Baba C Vemuri, "Shape modeling with front propagation: A level set approach," IEEE Trans. Pattern Anal. Mach. Intell., vol. 17, no. 2, pp. 158-175, 1995.

[95] Timothy F Cootes and Christopher J Taylor, "Active shape models: smart snakes," in Proceedings of the British Machine Vision Conference (BMVC'92), Leeds, UK, September 1992, pp. 266-275, Springer.

[96] F. Khalifa, G.M. Beache, A. Elnakib, H. Sliman, G. Gimel'farb, K.C. Welch, and A. El-Baz, "A new shape-based framework for the left ventricle wall segmentation from cardiac first-pass perfusion MRI," in Proceedings of the International Symposium on Biomedical Imaging (ISBI'13), San Francisco, California, USA, April 7-11 2013, pp. 41-44.

[97] Fahmi Khalifa, Ahmed Elnakib, GarthM. Beache, Georgy Gimel'farb, MohamedAbo El-Ghar, Rosemary Ouseph, Guela Sokhadze, Samantha Manning, Patrick McClure, and Ayman El-Baz, “3D kidney segmentation from ct images using a level set approach guided by a novel stochastic speed function," in Proceedings of the International Conference on Medical Image Computing and Computer-Assisted Intervention (MICCAI'11), Gabor Fichtinger, Anne Martel, and Terry Peters, Eds., Toronto, Canada, September 18-22 2011, vol. 6893 of Lecture Notes in Computer Science, pp. 587-594, Springer Berlin Heidelberg.

[98] F. Khalifa, G. Beache, Ayman El-Baz, and G. Gimel'farb, “Deformable model guided by stochastic speed with application in cine images segmentation," 
in Proceedings of the 17th IEEE International Conference on Image Processing (ICIP'10), Hong Kong, China, September 26-29 2010, pp. 1725-1728.

[99] F. Khalifa, A. El-Baz, G. Gimel'farb, R. Ouseph, and M.A. El-Ghar, "Shapeappearance guided level-set deformable model for image segmentation," in Proceedings of the 20th International Conference on Pattern Recognition (ICPR'10), Istanbul, Turkey, August 23-26 2010.

[100] Stanley Osher and James A Sethian, "Fronts propagating with curvaturedependent speed: algorithms based on Hamilton-Jacobi formulations," J. Comput. Phys., vol. 79, no. 1, pp. 12-49, 1988.

[101] Fuxing Wang, Jasjit Suri, and Aaron Fenster, "Segmentation of prostate from 3-D ultrasound volumes using shape and intensity priors in level set framework," in Proceedings of the 28th Annual International Conference of the IEEE Engineering in Medicine and Biology Society (EMBS'06), New York, New York, USA, August 30-September 3 2006, IEEE, pp. 2341-2344.

[102] Yiqiang Zhan and Dinggang Shen, "Automated segmentation of 3D US prostate images using statistical texture-based matching method," in Proceedings of the International Conference on Medical Image Computing and Computer-Assisted Intervention (MICCAI'03), Montreal, Quebec, Canada, November 15-18 2003, pp. 688-696, Springer.

[103] Lixin Gong, Sayan D Pathak, David R Haynor, Paul S Cho, and Yongmin Kim, "Parametric shape modeling using deformable superellipses for prostate segmentation," IEEE Trans. Med. Imaging, vol. 23, no. 3, pp. 340-349, 2004.

[104] Laurent Saroul, Olivier Bernard, Didier Vray, and Denis Friboulet, "Prostate segmentation in echographic images: a variational approach using deformable super-ellipse and Rayleigh distribution," in Proceedings of the IEEE 
International Symposium on Biomedical Imaging: From Nano to Macro (ISBI'08), Paris, France, May 14-17 2008, IEEE, pp. 129-132.

[105] Sara Badiei, Septimiu E Salcudean, Jim Varah, and W James Morris, "Prostate segmentation in 2D ultrasound images using image warping and ellipse fitting," in Proceedings of the International Conference on Medical Image Computing and Computer-Assisted Intervention (MICCAI'06), Copenhagen, Denmark, October 1-6 2006, pp. 17-24, Springer.

[106] S Sara Mahdavi, Nick Chng, Ingrid Spadinger, William J Morris, and Septimiu E Salcudean, "Semi-automatic segmentation for prostate interventions," Med. Image Anal., vol. 15, no. 2, pp. 226-237, 2011.

[107] I.B. Tutar, S.D. Pathak, Lixin Gong, P.S. Cho, K. Wallner, and Yongmin Kim, "Semiautomatic 3-D prostate segmentation from TRUS images using spherical harmonics," IEEE Trans. Med. Imaging, vol. 25, no. 12, pp. 1645-1654, 2006.

[108] Guido Gerig, Martin Styner, D Jones, Daniel Weinberger, and Jeffrey Lieberman, "Shape analysis of brain ventricles using spherical harmonics," in IEEE Workshop on Mathematical Methods in Biomedical Image Analysis (MMBIA'01), Kauai, Hawaii, December 9-10 2001, IEEE, pp. 171-178.

[109] Ahmed Elnakib, Manuel F Casanova, Georgy Gimelrfarb, Andrew E Switala, and Ayman El-Baz, "Dyslexia diagnostics by 3-D shape analysis of the corpus callosum," IEEE Trans. Inf. Technol. in Biomed., vol. 16, no. 4, pp. 700-708, 2012.

[110] Fahmi Khalifa, Garth M Beache, Georgy Gimel'farb, Guruprasad A Giridharan, and Ayman El-Baz, "Accurate automatic analysis of cardiac cine images," IEEE Trans. Biomed. Eng., vol. 59, no. 2, pp. 445-455, 2012. 
[111] Ayman El-Baz, Ahmed Elnakib, Manuel F Casanova, Georgy Gimelfarb, Andrew E Switala, Desha Jordan, and Sabrina Rainey, "Accurate automated detection of autism related corpus callosum abnormalities," J. of Medical Syst., vol. 35, no. 5, pp. 929-939, 2011.

[112] Manuel F Casanova, Ayman El-Baz, Ahmed Elnakib, Jay Giedd, Judith M Rumsey, Emily L Williams, and Andrew E Switala, "Corpus callosum shape analysis with application to dyslexia," Transl. Neurosci., vol. 1, no. 2, pp. 124-130, 2010.

[113] Ahmed Elnakib, Matthew Nitzken, MF Casanova, H-Y Park, G Gimel'farb, and Ayman El-Baz, "Quantification of age-related brain cortex change using 3D shape analysis," in Proceedings of the International Conference on Pattern Recognition (ICPR'12), Tsukuba, Japan, November 11-15 2012, IEEE, pp. 4144.

[114] Ayman El-Baz, Matthew Nitzken, Fahmi Khalifa, Ahmed Elnakib, Georgy Gimel'farb, Robert Falk, and Mohammed Abo El-Ghar, "3D shape analysis for early diagnosis of malignant lung nodules," in Proceedings of theConference on Information Processing in Medical Imaging, (IPMI'11), 2011, pp. 772-783.

[115] Matthew Nitzken, MF Casanova, G Gimel'farb, Ahmed Elnakib, Fahmi Khalifa, A Switala, and Ayman El-Baz, "3D shape analysis of the brain cortex with application to dyslexia," in Proceedings of the IEEE International Conference on Image Processing (ICIP'11), Brussels, Belgium, September 11-14 2011, IEEE, pp. 2657-2660.

[116] Matthew Nitzken, MF Casanova, G Gimel'farb, Fahmi Khalifa, Ahmed Elnakib, Andrew E Switala, and Ayman El-Baz, "3D shape analysis of the brain cortex with application to autism," in Proceedings of the IEEE International 
Symposium on Biomedical Imaging: From Nano to Macro (ISBI'11), Chicago, Illinois, USA, March 30-April 2 2011, IEEE, pp. 1847-1850.

[117] A. Elnakib, Ayman El-Baz, M.F. Casanova, G. Gimel'farb, and A.E. Switala, "Image-based detection of corpus callosum variability for more accurate discrimination between dyslexic and normal brains," in Proceedings of the IEEE International Symposium on Biomedical Imaging: From Nano to Macro (ISBI'10), Rotterdam, Netherlands, April 14-17 2010, pp. 109-112.

[118] Ayman El-Baz and G Gimel'farb, "Robust image segmentation using learned priors," in Proceedings of the IEEE International Conference on Computer Vision (ICCV'09). IEEE, 2009, pp. 857-864.

[119] Ayman El-Baz and Georgy Gimel'farb, “Robust medical images segmentation using learned shape and appearance models," in Proccedings of the International Conference on Medical Image Computing and Computer-Assisted Intervention (MICCAI'09), London, UK, September 20-24 2009, pp. 281-288, Springer.

[120] Ayman El-Baz, Manuel Casanova, G Gimel'farb, Meghan Mott, A Switala, Eric Vanbogaert, and Russ McCracken, "Dyslexia diagnostics by 3D texture analysis of cerebral white matter gyrifications," in Proceedings of the International Conference on Pattern Recognition (ICPR'08), Tampa, Florida, USA, December 8-11 2008, IEEE, pp. 1-4.

[121] Ayman El-Baz, Manuel Casanova, G Gimel'farb, Meghan Mott, A Switala, Eric Vanbogaert, and Russ McCracken, "A new CAD system for early diagnosis of dyslexic brains," in Proceedings of the IEEE International Conference on Image Processing (ICIP'08), San Diego, California, USA, October 12-15 2008, IEEE, pp. 1820-1823. 
[122] Ayman El-Baz, Manuel F Casanova, Georgy Gimel'farb, Meghan Mott, and Andrew E Switwala, "A new image analysis approach for automatic classification of autistic brains," in Proceedings of the IEEE International Symposium on Biomedical Imaging: From Nano to Macro (ISBI'07), Washington, D.C., USA, April 12-16 2007, IEEE, pp. 352-355.

[123] Ayman El-Baz, Manuel F Casanova, Georgy Gimelfarb, Meghan Mott, and Andrew E Switala, "Autism diagnostics by 3D texture analysis of cerebral white matter gyrifications," in Proceedings of the International Conference on Medical Image Computing and Computer-Assisted Intervention (MICCAI'07), Brisbane, Australia, October 29-November 2 2007, pp. 882-890.

[124] Tim Cootes, ER Baldock, and J Graham, "An introduction to active shape models," Image Process. Anal., pp. 223-248, 2000.

[125] Dinggang Shen, Yiqiang Zhan, and Christos Davatzikos, "Segmentation of prostate boundaries from ultrasound images using statistical shape model," IEEE Trans. Med. Imaging, vol. 22, no. 4, pp. 539-551, 2003.

[126] Dennis Gabor, "Theory of communication," J. Inst. Electr. Eng., vol. 93, no. 26, pp. 429-441, 1946.

[127] Nacim Betrouni, Maximilien Vermandel, David Pasquier, Salah Maouche, and Jean Rousseau, "Segmentation of abdominal ultrasound images of the prostate using a priori information and an adapted noise filter," Comput. Med. Imaging Graph., vol. 29, no. 1, pp. 43-51, 2005.

[128] Amjad Zaim and Jerzy Jankun, "An energy-based segmentation of prostate from ultrasouind images using dot-pattern select cells," in Proceedings of the IEEE International Conference on Acoustics, Speech and Signal Processing (ICASSP 2007). IEEE, 2007, vol. 1, pp. 297-300. 
[129] Pingkun Yan, Sheng Xu, Baris Turkbey, and Jochen Kruecker, "Discrete deformable model guided by partial active shape model for TRUS image segmentation," IEEE Trans. Biomed. Eng., vol. 57, no. 5, pp. 1158-1166, 2010.

[130] Tony Chan and Wei Zhu, "Level set based shape prior segmentation," in Proceedings of the IEEE Computer Society Conference on Computer Vision and Pattern Recognition (CVPR'05), San Diego, California, USA, June 20-26 2005, IEEE, vol. 2, pp. 1164-1170.

[131] Adam C Hodge, Aaron Fenster, Dónal B Downey, and Hanif M Ladak, "Prostate boundary segmentation from ultrasound images using 2D active shape models: Optimisation and extension to 3D," Comput. Methods and Progr. Biomed., vol. 84, no. 2, pp. 99-113, 2006.

[132] Timothy F. Cootes, Gareth J. Edwards, and Christopher J. Taylor, "Active appearance models," IEEE Trans. Pattern Anal. Mach. Intell., vol. 23, no. 6, pp. 681-685, 2001.

[133] Soumya Ghose, Arnau Oliver, Robert Martí, Xavier Lladó, Jordi Freixenet, Joan C Vilanova, and Fabrice Meriaudeau, "Texture guided active appearance model propagation for prostate segmentation," in Prostate Cancer Imaging. 2010, pp. 111-120, Springer.

[134] Radomir S Stanković and Bogdan J Falkowski, "The Haar wavelet transform: its status and achievements," Comput. Elec. Engineer., vol. 29, no. 1, pp. 25-44, 2003.

[135] Soumya Ghose, Arnau Oliver, Robert Martí, Xavier Lladó, Jordi Freixenet, Jhimli Mitra, Joan C Vilanova, Josep Comet, and Fabrice Meriaudeau, "Multiple mean models of statistical shape and probability priors for automatic prostate segmentation," in Prostate Cancer Imaging, pp. 35-46. Springer, 2011. 
[136] Ian Jolliffe, Principal component analysis, Wiley Online Library, 2005.

[137] R Medina, A Bravo, P Windyga, J Toro, Pingkun Yan, and G Onik, “A 2-D active appearance model for prostate segmentation in ultrasound images," in Proceedings of the Annual International Conference of the Engineering in Medicine and Biology Society (EMBS'05), Shanghai, China, September 1-4 2006, IEEE, pp. 3363-3366.

[138] A. Soliman, F. Khalifa, A. Alansary, G. Gimel'farb, and A. El-Baz, "Segmentation of lung region based on using parallel implementation of joint MGRF: Validation on 3D realistic lung phantoms," in Proceedings of the IEEE International Symposium on Biomedical Imaging (ISBI'13), San Francisco, California, USA, April 7-11 2013, pp. 864-867.

[139] Behnoush Abdollahi, Ahmed Soliman, A.C. Civelek, X.-F. Li, G. Gimel'farb, and Ayman El-Baz, "A novel 3D joint MGRF framework for precise lung segmentation," in Proceedings of the Third International Workshop on Machine Learning in Medical Imaging, Nice, France, October 12012.

[140] A. Elnakib, G.M. Beache, M. Nitzken, G. Gimel'farb, and A. El-Baz, "A new framework for automated identification of pathological tissues in contrast enhanced cardiac magnetic resonance images," in Proceedings of the IEEE International Symposium on Biomedical Imaging: From Nano to Macro (ISBI'11), Chicago, Illinois, USA, March 30-April 2 2011, pp. 1272-1275.

[141] Ayman El-Baz, Georgy L. Gimel'farb, Robert Falk, Trevor Holland, and Teresa Shaffer, "A framework for unsupervised segmentation of lung tissues from low dose computed tomography images.," in Proceedings of the British Machine Vision Conference (BMVC'08), Mark Everingham, Chris J. Needham, and Roberto Fraile, Eds., Leeds, UK, September 1-4 2008, British Machine Vision Association. 
[142] Ayman El-Baz, Georgy Gimel'farb, Robert Falk, Trevor Holland, and Teresa Shaffer, "A new stochastic framework for accurate lung segmentation," in Proceedings of the International Conference on Medical Image Computing and Computer-Assisted Intervention (MICCAI'08), New York, New York, USA, September 6-10 2008.

[143] A. Firjani, A. Elmaghraby, and A. El-Baz, "MRI-based diagnostic system for early detection of prostate cancer," in Proceedings of the Conference on Biomedical Sciences and Engineering Conference (BSEC'13), Oak Ridge, Tennesse, USA, May 21-23 2013, pp. 1-4.

[144] A. Elnakib, G. Gimel'farb, T. Inanc, and A. El-Baz, “Modified Akaike information criterion for estimating the number of components in a probability mixture model," in Proceedings of the IEEE International Conference on Image Processing (ICIP'12), Orlando, Florida, USA, September 30-October 3 2012, pp. 2497-2500.

[145] A. Firjani, F. Khalifa, A. Elnakib, G. Gimel'farb, M. Abou El-Ghar, A. Elmaghraby, and A. El-Baz, "A novel image-based approach for early detection of prostate cancer," in Proceedings of the IEEE International Conference on Image Processing (ICIP'12), Orlando, Florida, USA, September 30-October 3 2012, pp. 2849-2852.

[146] A. Firjani, F Khalifa, A Elnakib, G. Gimmel'farb, M. Abo El-Ghar, A. Elmagharby, and A. El-Baz, "3D automatic approach for precise segmentation of the prostate from diffusion-weighted magnetic resonance imaging," in Proceedings of the IEEE International Conference on Image Processing (ICIP'11), Brussels, Belgium, September 11-14 2011.

[147] Ahmad Firjany, Ahmed Elnakib, Ayman El-Baz, Georgy Gimel'farb, Mohamed El-Ghar, and Adel Elmagharby, "Novel stochiastic framework for 
accurate segmentation of prostate in dynamic contrast enhanced MRI," in Proceedings of the First International Workshop on Prostate Cancer Imaging: Computer-Aided Diagnosis, Prognosis, and Intervention, Beijing, China, September 24 2010, vol. 1, pp. 123-130.

[148] Yuri Boykov and Olga Veksler, "Graph cuts in vision and graphics: Theories and applications," in Handbook of Mathematical Models in Computer Vision, pp. 79-96. Springer, 2006.

[149] SS Mohamed, AM Youssef, EF El-Saadany, and Magdy MA Salama, "Prostate tissue characterization using TRUS image spectral features," in Image Anal. Recognit., pp. 589-601. Springer, 2006.

[150] Soumya Ghose, Arnau Oliver, Robert Marti, Xavier Lladó, Jordi Freixenet, Jhimli Mitra, Joan Carles Vilanova, Josep Comet, and Fabrice Meriaudeau, "Statistical shape and probability prior model for automatic prostate segmentation," in Proceedings of the International Conference on Digital Image Computing Techniques and Applications (DICTA'11). IEEE, 2011, pp. 340-345.

[151] Seok Min Han, Hak Jong Lee, and Jin Young Choi, "Computer-aided prostate cancer detection using texture features and clinical features in ultrasound image," J. Digit. Imaging, vol. 21, no. 1, pp. 121-133, 2008.

[152] Rafael Llobet, Juan C Pérez-Cortés, Alejandro H Toselli, and Alfons Juan, "Computer-aided detection of prostate cancer," Int. J. Med. Inf., vol. 76, no. 7, pp. 547-556, 2007.

[153] Simona Maggio, M Alessandrini, L De Marchi, and Nicolo Speciale, "Computer aided detection of prostate cancer based on GDA and predictive deconvolution," in Proceedings of the IEEE Ultrasonics Symposium (IUS'08), Beijing, China, November 2-5 2008, IEEE, pp. 28-31. 
[154] Simona Maggio, Alessandro Palladini, Luca De Marchi, Martino Alessandrini, Nicolo Speciale, and Guido Masetti, "Predictive deconvolution and hybrid feature selection for computer-aided detection of prostate cancer," IEEE Trans. Med. Imaging, vol. 29, no. 2, pp. 455-464, 2010.

[155] M Scebran, A Palladini, S Maggio, L De Marchi, and N Speciale, "Automatic regions of interest segmentation for computer aided classification of prostate TRUS images," in Acoust. Imaging, pp. 285-293. Springer, 2011.

[156] Chengjun Sun and William G Wee, "Neighboring gray level dependence matrix for texture classification," Comput. Vis., Graph., Image Process., vol. 23, no. 3, pp. 341-352, 1983.

[157] SS Mohamed, MMA Salama, M Kamel, EF El-Saadany, K Rizkalla, and J Chin, "Prostate cancer multi-feature analysis using trans-rectal ultrasound images," Phys. Med. Biol., vol. 50, no. 15, pp. N175, 2005.

[158] SS Mohamed, Magdy MA Salama, Mohamed Kamel, and K Rizkalla, "Region of interest based prostate tissue characterization using least square support vector machine (LS-SVM)," in Image Anal. Recognit., pp. 51-58. Springer, 2004.

[159] Richard W Conners and Charles A Harlow, "A theoretical comparison of texture algorithms," IEEE Trans. Pattern Anal. Mach. Intell., , no. 3, pp. 204$222,1980$.

[160] L Masotti, E Biagi, S Granchi, L Breschi, E Magrini, and F Di Lorenzo, “Clinical test of RULES," in Proceedings of the IEEE Ultrasonics Symposium (IUS'04), Montreal, Quebec, Canada, August 24-27 2004, IEEE, vol. 3, pp. 2173-2176.

[161] Thomas Wagner, "Texture analysis," Handbook of computer vision and applications, vol. 2, pp. 275-308, 1999. 
[162] Ernest J Feleppa, William R Fair, Tian Liu, Andrew Kalisz, William Gnadt, Frederick L Lizzi, KC Balaji, Christopher R Porter, and Harold Tsai, "Twodimensional and three-dimensional tissue-type imaging of the prostate based on ultrasonic spectrum analysis and neural network classification," in Proceedings of the SPIE Conference on Medical Imaging 2000, San Diego, California, USA, February 13-17 2000, International Society for Optics and Photonics, pp. 152-160.

[163] Robert Roy, Arogyaswami Paulraj, and Thomas Kailath, "ESPRIT-A subspace rotation approach to estimation of parameters of cisoids in noise," IEEE Trans. Acoust., Speech and Signal Process., vol. 34, no. 5, pp. 1340-1342, 1986.

[164] Richard Roy and Thomas Kailath, "ESPRIT-Estimation of signal parameters via rotational invariance techniques," IEEE Trans. Acoust., Speech and Signal Process., vol. 37, no. 7, pp. 984-995, 1989.

[165] Geoffrey McLachlan, Discriminant analysis and statistical pattern recognition, John Wiley and Sons, 2004.

[166] Ian Chan, William Wells III, Robert V Mulkern, Steven Haker, Jianqing Zhang, Kelly H Zou, Stephan E Maier, and Clare MC Tempany, “Detection of prostate cancer by integration of line-scan diffusion, T2-mapping and T2weighted magnetic resonance imaging; a multichannel statistical classifier," Med. Phys., vol. 30, pp. 2390, 2003.

[167] Richard O Duda, Peter E Hart, and David G Stork, Pattern classification, John Wiley \& Sons, 2012.

[168] Michael E Tipping, "Sparse Bayesian learning and the relevance vector machine," J. Mach. Learn. Res., vol. 1, pp. 211-244, 2010. 
[169] David A Clausi and ME Jernigan, “Designing Gabor filters for optimal texture separability," Pattern Recognit., vol. 33, no. 11, pp. 1835-1849, 2000.

[170] Anil K Jain and Farshid Farrokhnia, "Unsupervised texture segmentation using Gabor filters," Pattern Recognit., vol. 24, no. 12, pp. 1167-1186, 1991.

[171] Samar S Mohamed and Magdy MA Salama, "Prostate cancer spectral multifeature analysis using trus images," IEEE Trans. on Med. Imaging, vol. 27, no. 4, pp. 548-556, 2008.

[172] Yanong Zhu, Stuart Williams, and Reyer Zwiggelaar, "A hybrid ASM approach for sparse volumetric data segmentation," Pattern Recognit. Image Anal., vol. 17, no. 2, pp. 252-258, 2007.

[173] Robert Toth, Pallavi Tiwari, Mark Rosen, Arjun Kalyanpur, Sona Pungavkar, and Anant Madabhushi, "A multimodal prostate segmentation scheme by combining spectral clustering and active shape models," in Proceedings of the SPIE Conference on Medical Imaging 2008, San Diego, California, USA, February 16-21 2008, pp. 1-12.

[174] Yi Gao, Romeil Sandhu, Gabor Fichtinger, and Allen Robert Tannenbaum, "A coupled global registration and segmentation framework with application to magnetic resonance prostate imagery," IEEE Trans. Med. Imaging, vol. 29, no. 10, pp. 1781-1794, 2010.

[175] Soumya Ghose, Arnau Oliver, Robert Martí, Xavier Lladó, Jordi Freixenet, Jhimli Mitra, Joan C Vilanova, and Fabrice Meriaudeau, "A hybrid framework of multiple active appearance models and global registration for 3D prostate segmentation in MRI," in Proceedings of the SPIE Conference on Medical Imaging 2012, February 4-9 2012, p. 83140S. 
[176] Sébastien Martin, Jocelyne Troccaz, and Vincent Daanen, "Automated segmentation of the prostate in 3D mr images using a probabilistic atlas and a spatially constrained deformable model," Med. Phys., vol. 37, pp. 1579, 2010.

[177] Philip D Allen, James Graham, David C Williamson, and Charles E Hutchinson, "Differential segmentation of the prostate in MR images using combined 3D shape modelling and voxel classification," in Proceedings of the 3rd IEEE International Symposium on Biomedical Imaging: (ISBI'06), Arlington, VIrginia, USA, April 6-9 2006, IEEE, pp. 410-413.

[178] Xin Liu, DL Langer, MA Haider, TH Van der Kwast, AJ Evans, MN Wernick, and IS Yetik, "Unsupervised segmentation of the prostate using MR images based on level set with a shape prior," in Proceedings of the Annual International Conference of the IEEE Engineering in Medicine and Biology Society (EMBC'09), Minneapolis, Minnesota, USA, September 2-6 2009, IEEE, pp. 3613-3616.

[179] Xin Liu, Masoom A Haider, and Imam Samil Yetik, "Unsupervised 3D prostate segmentation based on diffusion-weighted imaging MRI using active contour models with a shape prior," J. Electr. Comput. Eng., vol. 2011, pp. 11, 2011.

[180] Leo Grady, "Random walks for image segmentation," IEEE Trans. Pattern Anal. Mach. Intell., vol. 28, no. 11, pp. 1768-1783, 2006.

[181] Soumya Ghose, Jhimli Mitra, Arnau Oliver, Robert Marti, Xavier Llado, Jordi Freixenet, Joan Carles Vilanova, Désiré Sidibé, and Fabrice Mériaudeau, "Graph cut energy minimization in a probabilistic learning framework for 3D prostate segmentation in MRI," in Proceedings of the 21st International Conference on Pattern Recognition (ICPR'12), Tsukuba Science City, Japan, November 11-15 2012, IEEE, pp. 125-128. 
[182] A. Alansary, A. Soliman, F. Khalifa, A. Elnakib, M. Mostapha, M. Nitzken, M. Casanova, and A. El-Baz, "MAP-based framework for segmentation of MR brain images based on visual appearance and prior shape," MIDAS J., vol. 1, pp. 1-13, 2013.

[183] F. Khalifa, M. Abou El-Ghar, Behnaz Abdollahi, Hermann Frieboes, Tarek El-Diasty, and A. El-Baz, "A comprehensive non-invasive framework for automated evaluation of acute renal transplant rejection using DCE-MRI," NMR Biomed., vol. 26, no. 11, pp. 1460-1470, 2013.

[184] F. Khalifa, G. M. Beache, M. Abou El-Ghar, T. El-Diasty, G. Gimel'farb, M. Kong, and A. El-Baz, "Dynamic contrast-enhanced MRI-based early detection of acute renal transplant rejection," IEEE Trans. Med. Imaging, vol. 32, no. 10, pp. 1910-1927, 2013.

[185] Hisham Sliman, Fahmi Khalifa, Ahmed Elnakib, Ahmed Soliman, Ayman El-Baz, Garth M. Beache, Adel Elmaghraby, and Georgy Gimel'farb, “Myocardial borders segmentation from cine mr images using bidirectional coupled parametric deformable models," Med. Phys., vol. 40, no. 9, pp. 1-13, 2013.

[186] F. Khalifa, G. M. Beache, G. Gimel'farb, G. A. Giridharan, and A. El-Baz, "Accurate automatic analysis of cardiac cine images," IEEE Trans. Biomed. Eng., vol. 59, no. 2, pp. 445-455, 2012.

[187] A. Elnakib, G. M. Beache, G. Gimel'farb, and A. El-Baz, “New automated Markov-Gibbs random field based framework for myocardial wall viability quantification on agent enhanced cardiac magnetic resonance images," Int. J. Cardiovasc. Imaging, vol. 28, no. 7, pp. 1683-1698, 2012.

[188] A. El-Baz, G. Gimel'farb, R. Falk, and M. Abo El-Ghar, “Automatic analy- 
sis of 3D low dose CT images for early diagnosis of lung cancer," Pattern Recognit., vol. 42, no. 6, pp. 1041-1051, 2009.

[189] Aly A Farag, Ayman S El-Baz, and Georgy Gimel'farb, "Precise segmentation of multimodal images," IEEE Trans. Image Process., vol. 15, no. 4, pp. 952-968, 2006.

[190] H Sliman, F Khalifa, A Elnakib, A Soliman, GM Beache, A Elmaghraby, and A El-Baz, "A new segmentation-based tracking framework for extracting the left ventricle cavity from cine cardiac MRI," in Proceedings of the IEEE Conference on Image Processing (ICIP'13), Melbourne, Australia, September 15-18 2013, IEEE, pp. 685-689.

[191] A. Elnakib, G. M. Beache, M. Nitzken, G. Gimel'farb, and A. El-Baz, “A new framework for automated identification of pathological tissues in contrast enhanced cardiac magnetic resonance images," in Proceedings of the IEEE International Symposium on Biomedical Imaging: From Nano to Macro (ISBI'11), Chicago, Illinois, USA, March 30-April 2 2011, IEEE, pp. 1272-1275.

[192] A. El-Baz, G. Gimel'farb, R. Falk, M. Abou El-Ghar, V. Kumar, and D. Heredia, "A novel 3D joint Markov-Gibbs model for extracting blood vessels from PC-MRA images," in Proc. of the International Conference on Medical Image Computing and Computer-Assisted Intervention (MICCAI'09), London, UK, September 20-24 2009, pp. 943-950.

[193] A. El-Baz and G. Gimel'farb, “Robust image segmentation using learned priors," in Proceedings of the International Conference on Computer Vision (ICCV'09), Kyoto, Japan, September 27-October 4 2009, IEEE, pp. 857-864.

[194] A. El-Baz and G. Gimel'farb, “Robust medical images segmentation using learned shape and appearance models," in Proceedings of the Interna- 
tional Conference on Medical Image Computing and Computer-Assisted Intervention (MICCAI'09), London, UK, September 20-24 2009, pp. 281-288, Springer.

[195] Ayman El-Baz, Aly Farag, and Georgy Gimel'farb, “MGRF controlled stochastic deformable model," in Proceedings of the Scandinavian Conference on Image Analysis (SCIA'05), Joensuu, Finland, June 19-22 2005, pp. 1138-1147, Springer.

[196] A. El-Baz and A.A. Farag, "Image segmentation using GMRF models: parameters estimation and applications," in Proceedings of the 20th International Conference on Image Processing (ICIP'03), Barcelona, Catalonia, Spain, September 14-18 2003, vol. 2, pp. 177-80.

[197] A. El-Baz and G. Gimel'farb, "EM based approximation of empirical distributions with linear combinations of discrete Gaussians," in Proceedings of the International Conference on Image Processing (ICIP'07), San Antonio, Texas, USA, September 16-19 2007, IEEE, pp. 373-376.

[198] A. A. Farag, A. S. El-Baz, and G. Gimel'farb, "Precise segmentation of multimodal images," IEEE Trans. Image Process., vol. 15, no. 4, pp. 952-968, 2006.

[199] A. El-Baz, Novel Stochastic Models for Medical Image Analysis, Ph.D. thesis, University of Louisville, Louisville, KY, USA, 2006.

[200] A. El-Baz, A. Elnakib, F. Khalifa, M. Abou El-Ghar, R. Falk, and G. Gimel'farb, "Precise segmentation of 3-D magnetic resonance angiography," IEEE Trans. Biomed. Eng., vol. 59, no. 7, pp. 2019-2029, 2012.

[201] A. Elnakib, G. Gimel'farb, T. Inanc, and A. El-Baz, “Modified Akaike information criterion for estimating the number of components in a probability mixture model," in Proceedings of the IEEE International Conference on Image Processing (ICIP'12), Orlando, Plorida, USA, Sept 30-October 3 2013, pp. 2497-2500. 
[202] F. Khalifa, G. Gimel'farb, M.A. El-Ghar, G. Sokhadze, S. Manning, P. McClure, R. Ouseph, and A. El-Baz, "A new deformable model-based segmentation approach for accurate extraction of the kidney from abdominal CT images," in Proceedings of the IEEE International Conference on Image Processing (ICIP'11), Brussels, Belgium, September 11-14 2011, pp. 3393-3396.

[203] A. A. Farag, A. El-Baz, and G. Gimel'farb, “Density estimation using modified expectation-maximization algorithm for a linear combination of Gaussians," in Proceedings of the International Conference on Image Processing (ICIP'04), Singapore, October 24-27 2004, IEEE, vol. 3, pp. 1871-1874.

[204] A. Farag, A. El-Baz, and G. Gimel'farb, "Precise image segmentation by iterative EM-based approximation of empirical grey level distributions with linear combinations of Gaussians," in Proceedings of the IEEE Computer Vision and Pattern Recognition Workshop (CVPRW'04), Washington, D.C., USA, June 27-July 2 2004, IEEE, pp. 121-129.

[205] G. Gimel'farb, A. Farag, and A. El-Baz, "Expectation-maximization for a linear combination of Gaussians," in Proceedings of the International Conference on Pattern Recognition (ICPR'2004), Cambridge, UK, August 23-26 2004, pp. $422-425$.

[206] A. A. Farag, A. El-Baz, and R. M. Mohamed, "Density estimation using generalized linear model and a linear combination of Gaussians," Int. J. of Signal Process., vol. 1, pp. 76-79, 2005.

[207] A. El-Baz, R. M. Mohamed, A. A. Farag, and G. Gimel'farb, “Unsupervised segmentation of multi-modal images by a precise approximation of individual modes with linear combinations of discrete Gaussians," in Proceedings of the IEEE Computer Society Conference on Computer Vision and Pattern 
Recognition-Workshops (CVPRW'05), San Diego, California, USA, June 20-26 2005, IEEE, pp. 54-54.

[208] A. El-Baz, A. A. Farag, and G. Gimel'farb, "Iterative approximation of empirical grey-level distributions for precise segmentation of multimodal images," EURASIP J. Appl. Signal Process., vol. 2005, pp. 1969-1983, 2005.

[209] A. Firjani, A Elnakib, F. Khalifa, G. Gimmel'farb, M. Abo El-Ghar, J. Suri, A. Elmagharby, and A. El-Baz, "A new 3D automatic segmentation framework for accurate segmentation of prostate from DCE-MRI," in Proceedings of the IEEE International Conference on Image Processing (ICIP'11), Brussels, Belgium, September 11-14 2011.

[210] Ahmad Firjani, Ahmed Elnakib, Fahmi Khalifa, G Gimel'farb, M Abo ElGhar, J Suri, Adel Elmaghraby, and Ayman El-Baz, "A new 3D automatic segmentation framework for accurate segmentation of prostate from DCEMRI," in Proceedings of the IEEE International Symposium on Biomedical Imaging (ISBI'11), Chicago, Illinois, USA, March 40-April 2 2011, IEEE, pp. 1476-1479.

[211] Jianbo Shi and Jitendra Malik, "Normalized cuts and image segmentation," IEEE Trans. Pattern Anal. Mach. Intell., vol. 22, no. 8, pp. 888-905, 2000.

[212] Brian L Mark and William Turin, Probability, Random Processes, and Statistical Analysis, Cambridge University Press Textbooks, 2011.

[213] Nasr Makni, Philippe Puech, Renaud Lopes, Anne-Sophie Dewalle, Olivier Colot, and Nacim Betrouni, "Combining a deformable model and a probabilistic framework for an automatic 3D segmentation of prostate on MRI," Int. J. Comput. Assist. Radiol. Surg., vol. 4, no. 2, pp. 181-188, 2009.

[214] Stan Z Li and S Singh, Markov random field modeling in image analysis, vol. 3, Springer, 2009. 
[215] A. Firjani, A. Elnakib, F. Khalifa, G. Gimel'farb, M. Abo El-Ghar, A. Elmaghraby, and A. El-Baz, "A new 3D automatic segmentation framework for accurate extraction of prostate from diffusion imaging," in Proceedings of the Biomedical Sciences and Engineering Conference (BSEC'11), Knoxville, Tennesse, March 15-17 2011, pp. 1-4.

[216] Parmeshwar Khurd, Leo Grady, Kalpitkumar Gajera, Mamadou Diallo, Peter Gall, Martin Requardt, Berthold Kiefer, Clifford Weiss, and Ali Kamen, “Facilitating 3D spectroscopic imaging through automatic prostate localization in mr images using random walker segmentation initialized via boosted classifiers," in Prostate Cancer Imaging, pp. 47-56. Springer, 2011.

[217] Stefan Klein, Uulke A van der Heide, Irene M Lips, Marco van Vulpen, Marius Staring, and Josien PW Pluim, "Automatic segmentation of the prostate in 3D MR images by atlas matching using localized mutual information," Med. Phys., vol. 35, pp. 1407, 2008.

[218] Jason A Dowling, Jurgen Fripp, Shekhar Chandra, Josien P W Pluim, Jonathan Lambert, Joel Parker, James Denham, Peter B Greer, and Olivier Salvado, "Fast automatic multi-atlas segmentation of the prostate from 3D MR images," in Prostate Cancer Imaging, pp. 10-21. Springer, 2011.

[219] Thomas Robin Langerak, Uulke A van der Heide, Alexis NTJ Kotte, Max A Viergever, Marco van Vulpen, and Josien PW Pluim, “Label fusion in atlasbased segmentation using a selective and iterative method for performance level estimation (SIMPLE)," IEEE Trans. Med. Imaging, vol. 29, no. 12, pp. 2000-2008, 2010.

[220] Daniel Flores-Tapia, Gabriel Thomas, Niranjan Venugopal, Boyd McCurdy, and Stephen Pistorius, "Semi automatic MRI prostate segmentation based on wavelet multiscale products," in Proceedings of the 30th Annual International 
Conference of the IEEE Engineering in Medicine and Biology Society (EMBS'08), Vancouver, British Columbia, Canada, August 20-25 2008, IEEE, pp. 30203023.

[221] J-C Pesquet, Hamid Krim, and Hervé Carfantan, "Time-invariant orthonormal wavelet representations," IEEE Trans. Signal Process., vol. 44, no. 8, pp. 1964-1970, 1996.

[222] Siddharth Vikal, Steven Haker, Clare Tempany, and Gabor Fichtinger, "Prostate contouring in MRI guided biopsy," in Proc. SPIE Med. Imaging: Image Process., 2009, pp. 1-11.

[223] Anant Madabhushi, Michael D Feldman, Dimitris N Metaxas, John Tomaszeweski, and Deborah Chute, "Automated detection of prostatic adenocarcinoma from high-resolution ex vivo MRI," IEEE Trans. on Med. Imaging, vol. 24, no. 12, pp. 1611-1625, 2005.

[224] Satish Viswanath, B Nicolas Bloch, Elisabeth Genega, Neil Rofsky, Robert Lenkinski, Jonathan Chappelow, Robert Toth, and Anant Madabhushi, "A comprehensive segmentation, registration, and cancer detection scheme on 3 Tesla in vivo prostate DCE-MRI," in Proceedings of the Conference on Medical Image Computing and Computer-Assisted Intervention (MICCAI'08), New York, New York, USA, September 6-10, pp. 662-669.

[225] Pieter C Vos, Thomas Hambrock, Jelle O Barenstz, and Henkjan J Huisman, "Computer-assisted analysis of peripheral zone prostate lesions using T2weighted and dynamic contrast enhanced T1-weighted MRI," Phys. Med. Biol., vol. 55, no. 6, pp. 1719, 2010.

[226] Sedat Ozer, Masoom A Haider, Deanna L Langer, Theodorus H van der Kwast, Andrew J Evans, Miles N Wernick, John Trachtenberg, and Imam Samil Yetik, "Prostate cancer localization with multispectral MRI 
based on relevance vector machines," in IEEE International Symposium on Biomedical Imaging: From Nano to Macro (ISBI'2009), Boston, Massachusetts, USA, June 28-July 1 2009, IEEE, pp. 73-76.

[227] Anant Madabhushi, Jianbo Shi, Michael Feldman, Mark Rosen, and John Tomaszewski, "Comparing ensembles of learners: Detecting prostate cancer from high resolution MRI," in Computer Vision Approaches to Medical Image Analysis, pp. 25-36. Springer, 2006.

[228] R Lopes, A Ayache, N Makni, P Puech, A Villers, S Mordon, and N Betrouni, "Prostate cancer characterization on MR images using fractal features," Med. Phys., vol. 38, pp. 83, 2011.

[229] Yu Sub Sung, Heon-Ju Kwon, Bum-Woo Park, Gyunggoo Cho, Chang Kyung Lee, Kyoung-Sik Cho, and Jeong Kon Kim, "Prostate cancer detection on dynamic contrast-enhanced MRI: computer-aided diagnosis versus single perfusion parameter maps," Am. J. Roentgenol., vol. 197, no. 5, pp. 1122-1129, 2011.

[230] Klaus Engel, Markus Hadwiger, Joe M Kniss, Christof Rezk-Salama, and Daniel Weiskopf, Real-time volume graphics, AK Peters, Limited, 2006.

[231] Yoav Freund and Robert E. Schapire, "Experiments with a new boosting algorithm," in Proceedings of the International Conference on Machine Learning (ICML'96), Bari, Italy, July 3-6 1996, pp. 148-156.

[232] Paul S Tofts, Gunnar Brix, David L Buckley, Jeffrey L Evelhoch, Elizabeth Henderson, Michael V Knopp, Henrik BW Larsson, Ting-Yim Lee, Nina A Mayr, Geoffrey JM Parker, et al., “Estimating kinetic parameters from dynamic contrast-enhanced T1-weighted MRI of a diffusable tracer: standardized quantities and symbols," J. Magn. Reson. Imaging, vol. 10, no. 3, pp. 223-232, 1999. 
[233] Pieter C Vos, Thomas Hambrock, Jelle O Barenstz, and Henkjan J Huisman, "Automated calibration for computerized analysis of prostate lesions using pharmacokinetic magnetic resonance images," in Proceedings of the International Conference on Medical Image Computing and Computer-Assisted Intervention (MICCAI'09), London, UK, September 20-24 2009, pp. 836-843.

[234] Jeong Kon Kim, Seong Sook Hong, Young Jun Choi, Seong Ho Park, Hanjong Ahn, Choung-Soo Kim, and Kyoung-Sik Cho, "Wash-in rate on the basis of dynamic contrast-enhanced MRI: Usefulness for prostate cancer detection and localization," J. Magn. Reson. Imaging, vol. 22, no. 5, pp. 639-646, 2005.

[235] Philippe Puech, Nacim Betrouni, Romain Viard, Arnauld Villers, Xavier Leroy, and Laurent Lemaitre, "Prostate cancer computer-assisted diagnosis software using dynamic contrast-enhanced MRI," in Proceedings of the 29th Annual International Conference of the IEEE Engineering in Medicine and Biology Society (EMBS'07), Lyon, France, August 23-26 2007, IEEE, pp. 5567-5570.

[236] Philippe Puech, Nacim Betrouni, Nasr Makni, Anne-Sophie Dewalle, Arnauld Villers, and Laurent Lemaitre, "Computer-assisted diagnosis of prostate cancer using DCE-MRI data: design, implementation and preliminary results," Int. J. Comput. Assist. Radiol. Surg., vol. 4, no. 1, pp. 1-10, 2009.

[237] J Veltman, R Mann, T Kok, IM Obdeijn, N Hoogerbrugge, JG Blickman, and C Boetes, "Breast tumor characteristics of BRCA1 and BRCA2 gene mutation carriers on MRI," Eur. Radiol., vol. 18, no. 5, pp. 931-938, 2008.

[238] Iclal Ocak, Marcelino Bernardo, Greg Metzger, Tristan Barrett, Peter Pinto, Paul S Albert, and Peter L Choyke, "Dynamic contrast-enhanced MRI of prostate cancer at $3 \mathrm{~T}$ : a study of pharmacokinetic parameters," Am. J. Roentgenol., vol. 189, no. 4, pp. 192-201, 2007. 
[239] Sam T Roweis and Lawrence K Saul, “Nonlinear dimensionality reduction by locally linear embedding," Science, vol. 290, no. 5500, pp. 2323-2326, 2000.

[240] Marc R Engelbrecht, Henkjan J Huisman, Robert JF Laheij, Gerrit J Jager, Geert JLH van Leenders, Christina A Hulsbergen-Van De Kaa, Jean JMCH de la Rosette, Johan G Blickman, and Jelle O Barentsz, “Discrimination of prostate cancer from normal peripheral zone and central gland tissue by using dynamic contrast-enhanced MR imaging," Radiol., vol. 229, no. 1, pp. 248-254, 2003.

[241] Jeong Kon Kim, Seong Sook Hong, Young Jun Choi, Seong Ho Park, Hanjong Ahn, Choung-Soo Kim, and Kyoung-Sik Cho, "Wash-in rate on the basis of dynamic contrast-enhanced MRI: Usefulness for prostate cancer detection and localization," J. Magn. Reson. Imaging, vol. 22, no. 5, pp. 639-646, 2005.

[242] J. J. Fütterer, S. W. T. P. J. Heijmink, T. W. J. Scheenen, J. Veltman, H. J. Huisman, P. Vos, C. A. Hulsbergen-Van de Kaa, J. A. Witjes, P. F. M. Krabbe, A. Heerschap, and J. O. Barentsz, "Prostate cancer localization with dynamic contrast-enhanced MR imaging and proton MR spectroscopic imaging," $R a$ diology, vol. 241, no. 2, pp. 449-458, 2006.

[243] Olivier Rouvière, Olivier Valette, Stéphanie Grivolat, Catherine ColinPangaud, Raymonde Bouvier, Jean Yves Chapelon, Albert Gelet, and Denis Lyonnet, "Recurrent prostate cancer after external beam radiotherapy: Value of contrast-enhanced dynamic MRI in localizing intraprostatic tumorcorrelation with biopsy findings," Urology, vol. 63, no. 5, pp. 922-927, 2004.

[244] I. Ocak, M. Bernardo, G. Metzger, T. Barrett, P. Pinto, P. S. Albert, and P. L. Choyke, "Dynamic contrast-enhanced MRI of prostate cancer at 3T: A study of pharmacokinetic parameters," Am. J. Roentgenol., vol. 189, no. 4, pp. W192-W201, 2007. 
[245] Pieter C Vos, Thomas Hambrock, Jurgen J Fütterer, CA Hulsbergen-Van De Kaa, Jelle Barentsz, and Henkjan H Huisman, "Effect of calibration on computerized analysis of prostate lesions using quantitative dynamic contrast-enhanced magnetic resonance imaging," in Proceedings of the SPIE Conference on Medical Imaging. International Society for Optics and Photonics, 2007, pp. 65140U-65140U.

[246] Nobuyuki Otsu, "A threshold selection method from gray-level histograms," Automatica, vol. 11, no. 285-296, pp. 23-27, 1975.

[247] Alejandro F Frangi, Wiro J Niessen, Koen L Vincken, and Max A Viergever, "Multiscale vessel enhancement filtering," in Proceedings of the Conference on Medical Image Computing and Computer-Assisted Interventation (MICCAI'98), Cambridge, Massachusetts, USA, October 11-13 1998, pp. 130-137, Springer.

[248] A Firjani, F Khalifa, A Elnakib, G Gimel'farb, M Abo El-Ghar, A Elmaghraby, and A El-Baz, "Non-invasive image-based approach for early detection of prostate cancer," in Developments in E-systems Engineering (DeSE'11), Dubai, United Arab Emirates, December 6-8 2011, IEEE, pp. 172-177.

[249] Tsutomu Tamada, Teruki Sone, Yoshimasa Jo, Shinya Toshimitsu, Takenori Yamashita, Akira Yamamoto, Daigo Tanimoto, and Katsuyoshi Ito, “Apparent diffusion coefficient values in peripheral and transition zones of the prostate: comparison between normal and malignant prostatic tissues and correlation with histologic grade," J. Magn. Reson. Imaging, vol. 28, no. 3, pp. 720-726, 2008.

[250] Luis C Maas and Pratik Mukherjee, "Diffusion MRI: Overview and clinical applications in neuroradiology," Appl. Radiol., vol. 34, no. 11, pp. 44, 2005.

[251] D Ampeliotis, A Antonakoudi, K Berberidis, EZ Psarakis, and A Kounoudes, "A computer-aided system for the detection of prostate cancer based on 
magnetic resonance image analysis," in Proceedings of the 3rd International Symposium on Communications, Control and Signal Processing (ISCCSP'08), St. Julians, Malta, March 12-14 2008, IEEE, pp. 1372-1377.

[252] Masoom A Haider, Theodorus H van der Kwast, Jeff Tanguay, Andrew J Evans, Ali-Tahir Hashmi, Gina Lockwood, and John Trachtenberg, "Combined T2-weighted and diffusion-weighted MRI for localization of prostate cancer," Am. J. Roentgenol., vol. 189, no. 2, pp. 323-328, 2007.

[253] Xin Liu, Deanna L Langer, Masoom A Haider, Yongyi Yang, Miles N Wernick, and Imam Samil Yetik, "Prostate cancer segmentation with simultaneous estimation of Markov random field parameters and class," IEEE Trans. Med. Imaging, vol. 28, no. 6, pp. 906-915, 2009.

[254] Yusuf Artan, Masoom A Haider, Deanna L Langer, Theodorus H van der Kwast, Andrew J Evans, Yongyi Yang, Miles N Wernick, John Trachtenberg, and Imam Samil Yetik, "Prostate cancer localization with multispectral MRI using cost-sensitive support vector machines and conditional random fields," IEEE Trans. Image Process., vol. 19, no. 9, pp. 2444-2455, 2010.

[255] Daniel D Lee and H Sebastian Seung, "Learning the parts of objects by nonnegative matrix factorization," Nature, vol. 401, no. 6755, pp. 788-791, 1999.

[256] Farial Shahnaz, Michael W Berry, V Paul Pauca, and Robert J Plemmons, "Document clustering using nonnegative matrix factorization," Inform. Process. Manag., vol. 42, no. 2, pp. 373-386, 2006.

[257] Stefanos Zafeiriou, Anastasios Tefas, Ioan Buciu, and Ioannis Pitas, “Exploiting discriminant information in nonnegative matrix factorization with application to frontal face verification," IEEE T. Neural Networ., vol. 17, no. 3, pp. 683-695, 2006. 
[258] Yuchen Xie, Jeffrey Ho, and Baba C Vemuri, “Nonnegative factorization of diffusion tensor images and its applications," in Inform. Process. Med. Imaging. Springer, 2011, pp. 550-561.

[259] Roman Sandler and Michael Lindenbaum, "Nonnegative matrix factorization with earth mover's distance metric for image analysis," IEEE T. Pattern Anal., vol. 33, no. 8, pp. 1590-1602, 2011.

[260] Stanley Osher and Ronald Fedkiw, Level set methods and dynamic implicit surfaces, New York: Springer-Verlag, 2006.

[261] P. A. Viola and W. M. Wells III, "Alignment by maximization of mutual information," Int. J. Comput. Vision, vol. 24, no. 2, pp. 137-154, 1997.

[262] Ayman El-Baz, Ahmed Soliman, Patrick McClure, G Gimel'farb, Mohamed Abou El-Ghar, and Robert Falk, "Early assessment of malignant lung nodules based on the spatial analysis of detected lung nodules," in Proceedings of the IEEE International Symposioum on Biomedical Imaging: From Nana to Macro (ISBI'12). IEEE, 2012, pp. 1463-1466.

[263] Julian Besag, "Spatial interaction and the statistical analysis of lattice systems," Journal of the Royal Statistical Society. Series B (Methodological), pp. 192236, 1974.

[264] Michael W Berry, Murray Browne, Amy N Langville, V Paul Pauca, and Robert J Plemmons, "Algorithms and applications for approximate nonnegative matrix factorization," Computat. Stat. Data An., vol. 52, no. 1, pp. 155-173, 2007.

[265] Daniel D Lee and H Sebastian Seung, "Algorithms for non-negative matrix factorization," in Adv. Neural Inf. Process. Syst., 2000, pp. 556-562. 
[266] Aapo Hyvärinen, "Sparse code shrinkage: Denoising of nongaussian data by maximum likelihood estimation," Neural Comput., vol. 11, no. 7, pp. 1739$1768,1999$.

[267] Kolawole Oluwole Babalola, Brian Patenaude, Paul Aljabar, Julia Schnabel, David Kennedy, William Crum, Stephen Smith, Tim Cootes, Mark Jenkinson, and Daniel Rueckert, "An evaluation of four automatic methods of segmenting the subcortical structures in the brain," Neuroimage, vol. 47, no. 4, pp. 1435-1447, 2009.

[268] Solomon Kullback and Richard A Leibler, "On information and sufficiency," Ann. Math. Stat., pp. 79-86, 1951.

[269] Robi Polikar, "Ensemble based systems in decision making," IEEE Circuits Syst. Mag., vol. 6, no. 3, pp. 21-45, 2006.

[270] E. L. Williams, A. El-Baz, M. Nitzken, A. E. Switala, and M. F. Casanova, "Spherical harmonic analysis of cortical complexity in autism and dyslexia," Translat. Neurosci., vol. 3, no. 1, pp. 36-40, 2012.

[271] M. Nitzken, M. F. Casanova, G. Gimel'farb, A. Elnakib, F. Khalifa, A. Switala, and A. El-Baz, "3D shape analysis of the brain cortex with application to dyslexia," in Proc. IEEE Int. Conf. Image Process., (ICIP'11), 2011, pp. 26572660.

[272] M. F. Casanova, A. El-Baz, A. Elnakib, J. Giedd, J. M. Rumsey, E. L. Williams, and A. E. Switala, "Corpus callosum shape analysis with application to dyslexia," Translat. Neurosci., vol. 1, no. 2, pp. 124-130, 2010.

[273] A. Elnakib, A. El-Baz, M. F. Casanova, G. Gimel'farb, and A. E. Switala, "Image-based detection of corpus callosum variability for more accurate discrimination between dyslexic and normal brains," in Proc. IEEE Int. Symp. Biomed. Imaging: From Nano to Macro, (ISBI'10), 2010, pp. 109-112. 
[274] A. Elnakib, A. El-Baz, M. F. Casanova, and A. E. Switala, “Dyslexia diagnostics by centerline-based shape analysis of the corpus callosum," in Proc. Int. Conf. Pattern Recogni., (ICPR'10), 2010, pp. 261-264.

[275] A. El-Baz, M. Casanova, G. Gimel'farb, M. Mott, A. Switala, E. Vanbogaert, and R. McCracken, "Dyslexia diagnostics by 3D texture analysis of cerebral white matter gyrifications," in Proc. Int. Conf. Pattern Recogni., (ICPR'08), 2008, pp. 1-4.

[276] M. Nitzken, M. F. Casanova, F. Khalifa, G. Sokhadze, and A. El-Baz, "Shapebased detection of cortex variability for more accurate discrimination between autistic and normal brains," in Handbook of Multi Modality State-ofthe-Art Medical Image Segmentation and Registration Methodologies, A. El-Baz, R. Acharya, A. Laine, and J. Suri, Eds., chapter 7, pp. 161-185. Springer, 2011.

[277] A. Elnakib, M. F. Casanova, G. Gimel'farb, A. E. Switala, and A. El-Baz, "Autism diagnostics by centerline-based shape analysis of the corpus callosum," in Proceedings of the IEEE International Symposium on Biomedical Imaging: From Nano to Macro, (ISBI'11), 2011, pp. 1843-1846.

[278] A. El-Baz, A. Elnakib, M. F. Casanova, G. Gimel'farb, A. E. Switala, D. Jordan, and S. Rainey, "Accurate automated detection of autism related corpus callosum abnormalities," J. Med. Sys., vol. 35, no. 5, pp. 929-939, 2011.

[279] M. F. Casanova, A. El-Baz, A. Elnakib, A. E. Switala, E. L. Williams, D. L. Williams, N. J. Minshew, and T. E. Conturo, "Quantitative analysis of the shape of the corpus callosum in patients with autism and comparison individuals," Autism, vol. 15, no. 2, pp. 223-238, 2011.

[280] M. Nitzken, M. F. Casanova, G. Gimel'farb, F. Khalifa, A. Elnakib, A. E. Switala, and A. El-Baz, "3D shape analysis of the brain cortex with ap- 
plication to autism," in Proceedings of the IEEE International Symposium on Biomedical Imaging: From Nano to Macro, (ISBI'11), 2011, pp. 1847-1850.

[281] Manuel F Casanova, Brynn Dombroski, and Andrew E Switala, Imaging and the Corpus Callosum in Patients with Autism, Springer, 2014.

[282] Manuel F Casanova, Ayman S El-Baz, and Jasjit S Suri, Imaging the Brain in Autism, Springer.

[283] B. Dombroski, M. Nitzken, A. Elnakib, F. Khalifa, A. El-Baz, and M. F. Casanova, "Cortical surface complexity in a population-based normative sample," Transl. Neurosci., vol. 5, no. 1, pp. 1-8, 2014.

[284] M. F. Casanova, A. El-Baz, S. S. Kamat, B. A. Dombroski, F. Khalifa, A. Elnakib, A. Soliman, A. Allison-McNutt, and A. E. Switala, "Focal cortical dysplasias in autism spectrum disorders," Acta Neuropathol. Commun., vol. 1, no. 1, pp. 67, 2013.

[285] A. Elnakib, M. F. Casanova, G. Gimel'farb, and A. El-Baz, "Autism diagnostics by 3D shape analysis of the corpus callosum," in Machine Learning in Computer-aided Diagnosis: Medical Imaging Intelligence and Analysis, K. Suzuki, Ed., chapter 15, pp. 315-335. IGI Global, Berlin, 2012.

[286] M. Mostapha, F. Khalifa, A. Alansary, A. Soliman, J. Suri, and A. El-Baz, "Computer-aided diagnosis systems for acute renal transplant rejection: Challenges and methodologies," in Abdomen and Thoracic Imaging, A. El-Baz and L. saba J. Suri, Eds., pp. 1-35. Springer, 2014.

[287] A. Rudra, A. Chowdhury, A. Elnakib, F. Khalifa, A. Soliman, G. M. Beache, and A. El-Baz, "Kidney segmentation using graph cuts and pixel connectivity," Pattern Recogni. Letters, vol. 34, no. 13, pp. 1470-1475, 2013. 
[288] F. Khalifa, A. Elnakib, G. M. Beache, G. Gimel'farb, M. A. El-Ghar, G. Sokhadze, S. Manning, P. McClure, and A. El-Baz, “3D kidney segmentation from CT images using a level set approach guided by a novel stochastic speed function," in Proceedings of the Medical Image Computing and ComputerAssisted Intervention (MICCAI'11), Toronto, Canada, September 18-22 2011, pp. 587-594.

[289] F. Khalifa, A. El-Baz, G. Gimel'farb, and M. Abo El-Ghar, "Non-invasive image-based approach for early detection of acute renal rejection," in Proceedings of the Medical Image Computing and Computer-Assisted Intervention (MICCAI'10), 2010, pp. 10-18.

[290] F. Khalifa, A. El-Baz, G. Gimel'farb, R. Ouseph, and M. A. El-Ghar, "Shapeappearance guided level-set deformable model for image segmentation," in Proceedings of the Intertional Conference on Pattern Recognition (ICPR'10), 2010, pp. 4581-4584.

[291] A. El-Baz and Georgy Gimel'farb, "Robust medical images segmentation using learned shape and appearance models," in Proceedings of the Medical Image Computing and Computer-Assisted Intervention (MICCAI'09), 2009, pp. 281-288.

[292] A. El-Baz, G. Gimel'farb, and M. Abo El-Ghar, "A novel image analysis approach for accurate identification of acute renal rejection," in Proceedings of the IEEE International Conference on Image Processing (ICIP'08), San Diego, California, USA, October 12-15 2008, pp. 1812-1815.

[293] A. El-Baz, G. Gimel'farb, and M. Abo El-Ghar, "Image analysis approach for identification of renal transplant rejection," in Proceedings of the IEEE International Conference on Pattern Recognition (ICPR'08), San Diego, California, USA, October 12-15 2008, pp. 1-4. 
[294] A. El-Baz and G. Gimel'farb, “Image segmentation with a parametric deformable model using shape and appearance priors," in Proceedings of the IEEE International Conference on Computer Vision and Pattern Recognition (CVPR'08), Anchoarge, Alaska, USA, June 24-26 2008, pp. 1-8.

[295] A. El-Baz, A. A. Farag, S. E. Yuksel, M. E. A. El-Ghar, T. A. Eldiasty, and M. A. Ghoneim, "Application of deformable models for the detection of acute renal rejection," in Deformable Models, A. A. Farag and J. S. Suri, Eds., vol. 1, chapter 10, pp. 293-333. 2007.

[296] A. El-Baz, G. Gimel'farb, and M. Abou El-Ghar, “New motion correction models for automatic identification of renal transplant rejection," in Proceedings of the Medical Image Computing and Computer-Assisted Intervention (MICCAI'07), Brisbane, Australia, USA, October 29 - November 2 2007, pp. 235243.

[297] A. M. Ali, A. A. Farag, and A. El-Baz, "Graph cuts framework for kidney segmentation with prior shape constraints," in Proceedings of the Medical Image Computing and Computer-Assisted Intervention (MICCAI'07), Brisbane, Australia, USA, October 29 - November 2 2007, vol. 1, pp. 384-392.

[298] A. El-Baz, A. Farag, R. Fahmi, S. Yuksel, W. Miller, M. Abou El-Ghar, T. ElDiasty, and M. Ghoneim, "A new CAD system for the evaluation of kidney diseases using DCE-MRI," in Proceedings of the Medical Image Computing and Computer-Assisted Intervention (MICCAI'08), New York, New York, USA, September 6-10 2006, pp. 446-453.

[299] A. El-Baz, A. Farag, R. Fahmi, S. Yuksel, M. Abo El-Ghar, and T. Eldiasty, "Image analysis of renal DCE MRI for the detection of acute renal rejection," in Proceedings of the IEEE International Conference on Pattern Recognition (ICPR'06), Arlington, Virgina, USA, April 6-9 2006, pp. 822-825. 
[300] A. Farag, A. El-Baz, S. Yuksel, M. Abou El-Ghar, and T. Eldiasty, "A framework for the detection of acute rejection with dynamic contrast enhanced magnetic resonance imaging," in Proceedings of the IEEE International Symposium on Biomedical Imaging: From Nano to Macro (ISBI'06), Arlington, Virgina, USA, April 6-9 2006, pp. 418-421.

[301] Seniha E Yuksel, Ayman El-Baz, and Aly A Farag, "A kidney segmentation framework for dynamic contrast enhanced magnetic resonance imaging," in Proceedings of the International Symposium on Mathmatical Methods in Engineering, (MME'06), 2006, pp. 55-64.

[302] Seniha E. Yuksel, Ayman El-Baz, Aly A. Farag, M. E. Abo El-Ghar, Tarek A. Eldiasty, and Mohamed A. Ghoneim, "Automatic detection of renal rejection after kidney transplantation," in International Congress Series, 2005, vol. 1281, pp. 773-778.

[303] A El-Baz, Aly A Farag, Robert Falk, and Renato La Rocca, "Automatic identification of lung abnormalities in chest spiral CT scans," in Proceedings of the IEEE International Conference on Acoustic, Speech, and Signal Processing (ICASSP'03), Hong Kong, Hong Kong, April 6-10 2003, vol. 2, pp. 261-264.

[304] Ayman El-Baz, Aly A Farag, Robert Falk, and Renato La Rocca, "A unified approach for detection, visualization, and identification of lung abnormalities in chest spiral CT scans," in International Congress Series, 2003, vol. 1256, pp. 998-1004.

[305] Ayman El-Baz, Aly A Farag, Robert Falk, and Renato La Rocca, "Detection, visualization and identification of lung abnormalities in chest spiral CT scan: Phase-I," in Proceedings of the International Conference on Biomedical Engineering, 2002, pp. 38-42. 
[306] Aly A Farag, Ayman El-Baz, Georgy Gimel'farb, and Robert Falk, “Detection and recognition of lung abnormalities using deformable templates," in Proceedings of the International Conference on Pattern Recognition (ICPR'04), Cambridge, UK, August 23-26 2004, vol. 3, pp. 738-741.

[307] Aly A Farag, Ayman El-Baz, Georgy G Gimelfarb, Robert Falk, and Stephen G Hushek, "Automatic detection and recognition of lung abnormalities in helical CT images using deformable templates," in Proceedings of the Medical Image Computing and Computer-Assisted Intervention (MICCAI'04), pp. 856-864. Sint-Malo, France, September 26-29 2004. 


\section{CURRICULUM VITAE}

\section{Patrick McClure}

\section{Contact Information:}

Address: 903 1/2 W. 7th St., Corbin, KY 40701

E-mail: patrick.s.mcclure@gmail.com

Phone: 1-606-344-2116

\section{Education:}

- B.S. in Bioengineering, University of Louisville (2013)

- M.S. in Computer Science, University of Louisville (2014)

\section{Honors and Awards:}

- Barry M. Goldwater Scholarship (2012)

- Jerry and Pat Sturgen Academic Excellence Award (2013)

- University of Louisville Undergraduate Research Grant (2012)

- First Place in J. B. Speed School Undergraduate Research Competition (2012)

- University of Louisville Trustees Scholarship (2009-2013)

- Kentucky Educational Excellence Scholarship (2009-2013)

- Dean's Scholar (7 Semesters)

- Dean's List (1 Semesters) 


\section{Employment:}

- Research Assistant, Bioimaging Lab, University of Louisville, Louisville, KY (Jan. 2011 - June 2014)

- Supplemental Instruction Tutor (Calculus III), University of Louisville, Louisville, KY (Aug. 2013 - Dec. 2013)

- Electronic Data Interchange (EDI) Engineering Intern, Epic Systems, Verona, WI (Jan. 2013 - May 2013)

- Research Assistant, Vision Lab, Johns Hopkins University, Baltimore, MD (May 2012 - Aug. 2012)

- Teacher's Assistant (Calculus III), University of Louisville, Louisville, KY (Jan. 2012 - May 2012)

- Manufacturing Engineering Co-op, Boston Scientific Coporation, Spencer, IN (Aug. 2011 - Dec. 2011)

- Supplemental Instruction Tutor (Differential Equations), University of Louisville, Louisville, KY (May 2011 - July 2011)

- Supplemental Instruction Tutor (Calculus II), University of Louisville, Louisville, KY (Aug. 2010 - Dec. 2010)

\section{Academic Interests:}

- Machine Learning

- Computer Vision

- Medical Image Analysis 


\section{Professional Societies:}

- Institute of Electrical and Electronics Engineers (IEEE)

- Biomedical Engineering Society (BMES)

- Tau Beta Pi Engineering Honors Society

\section{Publications:}

\section{Journal}

- A. El-Baz, A. Elnakib, F. Khalifa, M. Abou El-Ghar, P. McClure, Ahmed Soliman, and G. Gimel'farb, Precise Segmentation of 3D Magnetic Resonance Angiography, IEEE Transactions on Biomedical Engineering, 2012, pp. 2019-2029.

\section{Conference}

- A. Jain, L. Zappella, P. McClure, R. Vidal, Visual Dictionary Learning for Object Categorization and Segmentation, European Conference for Computer Vision (ECCV 2012), 2012, pp. 718-731.

- A. El-Baz, F. Khalifa, A. Elnakib, M. Nitzken, A. Soliman, P. McClure, M. Abo El-Ghar, G. Gimel'farb, A Novel Approach for Global Lung Registration Using 3D Markov-Gibbs Appearance Model, Proc. International Conference on Medical Image Computing and Computer Assisted Intervention (MICCAI 2012), 2012, pp. 114-121.

- A. El-Baz, F. Khalifa, A. Elnakib, M. Nitzken, A. Soliman, P. McClure, M. Abo El-Ghar, G. Gimel'farb, A Novel Approach for Global Lung Registration Using 3D Markov-Gibbs Appearance Model, Proc. International Conference on Medical Image Computing and Computer Assisted Intervention (MICCAI 2012), 2012, pp. 114-121. 
- F. Khalifa, G. Sokhadze, S. Manning, P. McClure, G. Gimelfarb, M. Abo El-Ghar, R. Ouseph, and A. El-Baz ," 3D Kidney Segmentation from CT Images Using Level Set Approach Guided by a Novel Stochastic Speed Function," Proc. International Conference on Medical Image Computing and Computer Assisted Intervention (MICCAI 2011), 2011, pp. 569-577.

- F. Khalifa, G. Sokhadze, S. Manning, P. McClure, G. Gimelfarb, M. Abo El-Ghar, R. Ouseph, and A. El-Baz , "A New Deformable Model-based Segmentation Approach for Accurate Extraction of the Kidney from Abdominal CT Images," Proc. International Conference on Image Processing (ICIP 11), 2011, pp. 3393 - 3396. 